\title{
Módulos tipo Verma sobre a álgebra TKK afim estendida
}

\author{
Anliy Natsuyo Nashimoto Sargeant
}

\author{
TESE APRESENTADA \\ AO \\ INSTITUTO DE MATEMÁTICA E ESTATÍSTICA \\ DA \\ UNIVERSIDADE DE SÃO PAULO \\ PARA \\ OBTENÇÃO DO TÍTULO DE DOUTOR \\ EM \\ CIÊNCIAS
}

Área de Concentração: Matemática

Orientador: Prof. Dr. Vyacheslav Futorny

Durante a elaboração deste trabalho a autora recebeu o auxílio financeiro da FAPESP

São Paulo

2007 


\section{Módulos tipo Verma sobre \\ a álgebra TKK afim estendida}

Este exemplar corresponde à redação final da tese devidamente corrigida e defendida por Anliy Natsuyo Nashimoto Sargeant e aprovada pela Comissão Julgadora.

São Paulo, 30 de março de 2007.

Banca examinadora:

- Prof. Dr. Vyacheslav Futorny (orientador) - IME/USP

- Prof. Dr. Ivan Shestakov - IME/USP

- Prof. Dr. Plamen Emilov Koshlukov - IMECC/UNICAMP

- Profa. Dra. Marinês Guerreiro - DMA/UFV

- Prof. Dr. Adriano Adrega de Moura - IMECC/UNICAMP 
Dedico esta tese a Adam e Aili. 
"A mente que se abre a uma nova idéia jamais voltará ao seu tamanho original."

Albert Einstein 


\section{Agradecimentos}

Agradeço primeiramente meu orientador, professor Vyacheslav Futorny, pela confiança, amizade, paciência e dedicação.

Agradeço o professor Ben Cox do College of Charleston pela orientação e apoio durante minha visita a esta instituição.

Agradeço as professoras Mary Lilian Lourenço, Iracema Martim Bund e Cristina Barufi pelo apoio e orientação no período em que cursava a especialização no IME-USP.

Agradeço a todos os professores do IME-USP que tive a satisfação de estudar e principalmente aos que me incentivaram desde o ínicio nessa caminhada.

Agradeço a todos os amigos do IME-USP pelo companheirismo.

Agradeço minha família pelo apoio em mais uma etapa da minha vida.

Agradeço a FAPESP por ter financiado este projeto. 


\section{Resumo}

As álgebras TKK afins estendidas pertencem à classe de álgebras de Lie chamada álgebras de Lie afins estendidas do tipo $A_{1}$. Elas são obtidas a partir de um semi-reticulado do $\mathbb{R}^{n}$. Estudamos a estrutura dos módulos tipo Verma sobre a álgebra TKK afim estendida para um semi-reticulado (não-reticulado) do $\mathbb{R}^{2}$. Quando fixamos um conjunto positivo de raízes isotrópicas chamado standard encontramos quatro órbitas da subálgebra de Borel que dão origem a distintos módulos tipo Verma sobre a álgebra TKK afim estendida. Estudamos as estruturas de seus submódulos e encontramos critérios de irredutibilidade para os módulos de Verma clássico e imaginário. 


\section{Abstract}

The extended affine TKK Lie algebras belong to a class of Lie algebras called extended affine Lie algebras of type $A_{1}$. They are obtained from a semilattice on $\mathbb{R}^{n}$. We studied the structure of the Verma type modules for the extended affine TKK algebra obtained from a semi-lattice (non-lattice) on $\mathbb{R}^{2}$. Fixing a set of positive isotropic roots called standard we found four orbits of the Borel subalgebra each of which give distinct Verma modules for the extended affine TKK algebra. We studied the structures of their submodules and found a criteria for irreducibility for the classic and imaginary Verma modules. 


\section{Sumário}

$\begin{array}{ll}\text { Introdução } & 1\end{array}$

1 Álgebras de Lie afins estendidas 4

1.1 Teoria básica das álgebras de Lie afins estendidas . . . . . . . . . . . . 4

1.1.1 Grupo de Weyl afim estendido . . . . . . . . . . . . . . . . . . 10

2 Álgebras TKK afins estendidas $\quad 11$

2.1 Semi-reticulados do $\mathbb{R}^{\nu} \ldots \ldots \ldots \ldots \ldots \ldots 11$

2.2 Semi-Reticulado do $\mathbb{R}^{2} \ldots \ldots \ldots \ldots \ldots$. . . . . . . . . . 19

3 Módulo de Verma Clássico $\quad 22$

3.1 Sistema de raízes positivo $\ldots \ldots \ldots \ldots \ldots \ldots \ldots$

3.2 Subálgebra de Kac-Moody afim . . . . . . . . . . . . . . . . 27

3.3 Base e Ordem . . . . . . . . . . . . . . . . . . . . . . . . . . . 28

3.4 Módulo de Verma Clássico . . . . . . . . . . . . . . . . . . . . . . . 30

3.5 Quociente integrável . . . . . . . . . . . . . . . . . 45

4 Módulo de Verma Imaginário $\quad 49$

4.1 Subálgebra tipo Heisenberg . . . . . . . . . . . . . . . . . . . . . 49

4.1.1 Módulo de Verma sobre a subálgebra tipo Heisenberg . . . . . . . . 50 


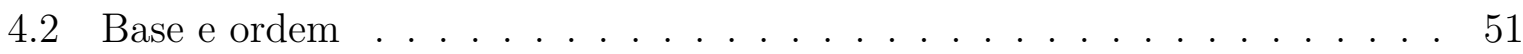

4.3 Módulo de Verma imaginário . . . . . . . . . . . . . . . . . . 53

4.4 Considerações finais . . . . . . . . . . . . . . . . . . . . . 61 


\section{Introdução}

As álgebras de Lie afins estendidas (ALAE), originalmente chamadas de álgebras de Lie quase-simples, foram introduzidas por Hoegh-Krohn e Torresani [9] em 1990 e sua teoria básica foi desenvolvida em [2]. Resumidamente, ela é uma álgebra de Lie sobre o corpo dos números complexos que possui: uma forma bilinear, simétrica, não-degenerada e invariante; uma subálgebra abeliana de dimensão finita, ad-diagonalizável, sendo seu próprio centralizador; e um sistema de raízes discreto e irredutível tal que os elementos dos subespaços das raízes não-isotrópicas são ad-nilpotentes.

A conjectura de Kac, provada em [2], dizia que a forma sobre a expansão real do sistema de raízes de uma ALAE é positivamente semi-definida. A ela associamos um sistema de raízes finito e definimos como sendo o tipo da álgebra o tipo do sistema de raízes finito: $A_{l}(l \geq 1), B_{l}(l \geq 2), C_{l}(l \geq 3), D_{l}(l \geq 4), E_{6}, E_{7}, E_{8}, F_{4}$ ou $G_{2}$, para o caso reduzido.

Nessa tese estudamos a ALAE do tipo $A_{1}$ que denominamos de álgebra TKK afim estendida.

No projeto de mestrado [16] um dos assuntos estudados foi o core de uma ALAE do tipo $A_{1}$, que é definido como sendo a subálgebra gerada pelo espaço de raízes nãoisotrópicas. Em [21], Yoshii descreve o core da ALAE do tipo $A_{1}$ como sendo isomorfo à extensão central da álgebra de Lie obtida de um torus de Jordan através da construção de Tits-Kantor-Koecher (TKK) e classifica todos os tori de Jordan, a menos de isomorfismo. Segundo o autor, um torus de Jordan sobre um corpo algebricamente fechado $F$ é isomorfo a um dos quatro Jordan tori (utilizamos a notação de [21]): $F_{q}^{+}$, onde $F_{q}$ é um torus quântico; $H\left(F_{\epsilon}, *\right)=\left\{x \in F_{\epsilon} \mid x^{*}=x\right\}$, onde $\epsilon$ a matriz quântica elementar, $F_{\epsilon}$ é um torus quântico e * uma involuçao sobre $F_{\epsilon} ; J_{S^{(m)}}$, torus de Clifford standard, onde $S^{(m)}$ é um semi-reticulado; ou $\mathbb{A}_{t}$, torus de Albert. 
A álgebra de Lie obtida de $F_{q}^{+}$através da construção de TKK é isormorfa a $s l_{2}\left(F_{q}\right)$. Quando $q$ é uma matriz quântica cujas entradas são todas iguais a 1 , temos $F_{q}^{+}=F_{q}$ a álgebra de polinômios de Laurent e a ALAE obtida dela é a álgebra de Lie toroidal do tipo $A_{1}$. A representação dessa álgebra foi estudada por diversos autores e destacamos aqui [3], [4] e [8] sobre módulos tipo Verma. Para $q$ uma matriz quântica qualquer, um estudo sobre o módulo de Verma imaginário sobre $s l_{2}\left(F_{q}\right)$ foi realizado em [5].

O torus de Clifford é obtido a partir de um semi-reticulado e de um torus de Clifford obtemos a álgebra TKK afim estendida. Em [19], Tan estuda a representação através de operadores de vertex da álgebra baby-TKK. Essa álgebra é obtida de um semi-reticulado (não reticulado) do $\mathbb{R}^{2}$ e, por ela possuir o menor sistema de raízes afim estendido [18] que não é do tipo finito e nem do tipo afim, o autor chamou tal álgebra de baby-TKK. A nossa ALAE é um quociente da baby-TKK estendida por derivações do grau e por isso resolvemos chamá-la de álgebra TKK afim estendida.

Estudamos nessa tese os módulos de Verma clássico e imaginário sobre a álgebra TKK afim estendida e os principais resultados obtidos foram critérios de irredutibilidade para ambos o módulos. No caso do módulo de Verma clássico, a subálgebra da álgebra TKK afim estendida que é isomorfa à álgebra de Kac-Moody afim $A_{1}^{(1)}$ tem papel fundamental no critério de irredutibilidade. No caso imaginário é a subálgebra tipo Heisenberg que irá deteminar a irredutibilidade.

No Capítulo 1 apresentamos os axiomas que definem uma ALAE e definimos o que é um sistema de raízes afim estendido, o tipo de uma ALAE e o grupo de Weyl afim estendido. Este capítulo foi baseado em [1] e[2].

No Capítulo 2 damos a definição de um semi-reticulado, obtemos uma álgebra de Jordan a partir dele e contruímos a álgebra TKK afim estendida. Apresentamos também alguns resultados sobre o grupo de Weyl afim estendido que são usados nos capítulos posteriores. A última seção desse capítulo dedicamos à álgebra TKK afim estendida obtida de um semi-reticulado (não-reticulado) do $\mathbb{R}^{2}$.

No Capítulo 3 definimos e apresentamos alguns resultados sobre o sistema de raízes positivo e classificamos, a menos de equivalência, os sistemas de raízes positivos quando fixado o conjunto positivo de raízes isotrópicas. De cada um deles obtemos distintos módulos tipo Verma, sendo um deles uma generalização do módulo de Verma clássico 
para o caso afim e o outro, a generalização do módulo de Verma imaginário. O principal resultado nesse capítulo é um critério de irredutibilidade: o módulo de Verma clássico sobre a álgebra TKK afim estendida é irredutível se, e somente se, o módulo de Verma sobre sua subálgebra isomorfo à álgebra $A_{1}^{(1)}$ é irredutível. Mostramos também que a álgebra TKK afim estendida não possui quocientes integráveis.

No Capítulo 4 tratamos do módulo de Verma imaginário e o principal resultado que provamos é que a irredutibilidade do módulo de Verma imaginário é determinada pelo módulo de Verma sobre a subálgebra tipo Heisenberg. Na última seção deste capítulo deixamos uma questão que surgiu baseada no trabalho [20]. A subálgebra tipo Heisenberg possui semelhanças com a álgebra Virasoro-like estudada em [20]. Nesse trabalho os autores provaram que quando a ordem é discreta, o módulo de Verma sobre a álgebra Virasoro-like é irredutível se, e somente se, o módulo de Verma sobre sua subálgebra (a álgebra de Heisenberg) é irredutivel. A ordem que nós definimos é discreta, no sentido de [20] e, a subálgebra tipo Heisenberg possui uma subálgebra que é a álgebra de Heisenberg. Portanto acreditamos que esse resultado também se aplica ao nosso caso. Isto implicaria que a irredutibilidade do módulo de Verma imaginário é determinada por uma subálgebra "menor". 


\section{Capítulo 1}

\section{Álgebras de Lie afins estendidas}

As álgebras de Lie afins estendidas são generalizações das álgebras de Kac-Moody afins e foram introduzidas em 1990 por R.Hoegh-Krohn e B.Torresani [9]. Os exemplos mais conhecidos de tais álgebras são as álgebras de Lie de dimensão finita, as álgebras de KacMoody afins e as álgebras de Lie toroidais. Um outro exemplo são as álgebras TKK afins estendidas que estudaremos na próxima seção. Essa seção foi baseada no trabalho [2] o qual introduziu a nomenclatura álgebras de Lie afins estendidas. Introduziremos aqui os axiomas que definem uma álgebra de Lie afim estendida, estudaremos seu sistema de raízes, definiremos o tipo da álgebra e de seu sistema de raízes e o grupo de Weyl afim estendido [1].

\subsection{Teoria básica das álgebras de Lie afins estendidas}

Seja $\mathcal{L}$ uma álgebra de Lie sobre $\mathbb{C}$ satisfazendo as seguintes propriedades:

(AE1) $\mathcal{L}$ possui uma forma bilinear, simétrica, não-degenerada e invariante

$$
(\cdot, \cdot): \mathcal{L} \times \mathcal{L} \longrightarrow \mathbb{C}
$$

A invariância é no sentido que

$$
([x, y], z)=(x,[y, z])
$$


para todo $x, y, z \in \mathcal{L}$.

(AE2) $\mathcal{L}$ possui uma subálgebra $\mathcal{H}$ abeliana, de dimensão finita, sendo o seu próprio centralizador em $\mathcal{L}$ e ad-diagonalizável, isto é, $a d_{\mathcal{L}} h$ é diagonalizável para todo $h \in \mathcal{H}$.

Para descrever as demais propriedades são necessárias mais algumas notações. Consideramos $\mathcal{H}^{*}$ o espaço dual complexo de $\mathcal{H}$ e para algum $\alpha \in \mathcal{H}^{*}$ definimos:

$$
\mathcal{L}_{\alpha}=\{x \in \mathcal{L} \mid[h, x]=\alpha(h) x, \text { para todo } h \in \mathcal{H}\}
$$

o espaço de raízes associado a $\alpha \in \mathcal{H}^{*}$. Definimos também:

$$
R=\left\{\alpha \in \mathcal{H}^{*} \mid \mathcal{L}_{\alpha} \neq 0\right\}
$$

o sistema de raízes de $\mathcal{L}$ com respeito a $\mathcal{H}$.

Devido à propriedade $(\mathrm{AE} 2)$ podemos considerar $\mathcal{L}$ como soma direta de $\mathcal{H}$-módulos

$$
\mathcal{L}=\bigoplus_{\alpha \in R} \mathcal{L}_{\alpha}
$$

Como o centralizador de $\mathcal{H}$ em $\mathcal{L}$ é o próprio $\mathcal{H}$ temos que

$$
\mathcal{L}_{0}=\mathcal{H}
$$

e como $\mathcal{H} \neq 0$ temos

$$
0 \in R
$$

Pela identidade de Jacobi obtemos a seguinte inclusão:

$$
\left[\mathcal{L}_{\alpha}, \mathcal{L}_{\beta}\right] \subseteq \mathcal{L}_{\alpha+\beta}, \text { para todo } \alpha, \beta \in \mathcal{H}^{*} .
$$

A invariância da forma em (AE1) implica que, para todo $\alpha, \beta \in \mathcal{H}^{*}$,

$$
\left(\mathcal{L}_{\alpha}, \mathcal{L}_{\beta}\right)=0 \text {, exceto se } \alpha+\beta=0 .
$$

Em particular, temos

$$
R=-R
$$

Mais ainda, a forma restrita a $\mathcal{H}$ é não-degenerada. De fato, considerando $h^{\prime} \in \mathcal{H}$ e $\left(h^{\prime}, h\right)=0$, para todo $h \in \mathcal{H}$, como $\left(\mathcal{L}_{\alpha}, \mathcal{L}_{0}\right)=0$ se $\alpha \neq 0$ implica que $\left(X, h^{\prime}\right)=0$ para todo $X \in \mathcal{L}_{\alpha}$ e $\alpha \neq 0$, como a forma $(\cdot, \cdot)$ é não-degenerada em $\mathcal{L}$ segue que $h^{\prime}=0$. 
Assim, como na teoria clássica de álgebras de Lie semi-simples complexas de dimensão finita, podemos transferir a forma para $\mathcal{H}^{*}$. Para cada $\alpha \in \mathcal{H}^{*}$, existe um único elemento $t_{\alpha} \in \mathcal{H}$ tal que

$$
\alpha(h)=\left(t_{\alpha}, h\right), \text { para todo } h \in \mathcal{H} .
$$

Logo, podemos transferir a forma para $\mathcal{H}^{*}$ definindo

$$
(\alpha, \beta)=\left(t_{\alpha}, t_{\beta}\right), \text { para todos } \alpha, \beta \in \mathcal{H}^{*}
$$

Para $\alpha \in \mathcal{H}^{*}, x_{\alpha} \in \mathcal{L}_{\alpha}, x_{-\alpha} \in \mathcal{L}_{-\alpha}$ e $h \in \mathcal{H}$ temos

$$
\begin{aligned}
\left(\left[x_{\alpha}, x_{-\alpha}\right], h\right) & =\left(x_{\alpha},\left[x_{-\alpha}, h\right]\right)=\alpha(h)\left(x_{\alpha}, x_{-\alpha}\right) \\
& =\left(t_{\alpha}, h\right)\left(x_{\alpha}, x_{-\alpha}\right)=\left(\left(x_{\alpha}, x_{-\alpha}\right) t_{\alpha}, h\right)
\end{aligned}
$$

e assim, como $\left[\mathcal{L}_{\alpha}, \mathcal{L}_{-\alpha}\right] \subseteq \mathcal{H}$, temos que

$$
\left[x_{\alpha}, x_{-\alpha}\right]=\left(x_{\alpha}, x_{-\alpha}\right) t_{\alpha} \text { e daí }\left[\mathcal{L}_{\alpha}, \mathcal{L}_{-\alpha}\right]=\mathbb{C} t_{\alpha}
$$

para $\alpha \in \mathcal{H}^{*}, x_{\alpha} \in \mathcal{L}_{\alpha}$ e $x_{-\alpha} \in \mathcal{L}_{-\alpha}$.

Agora podemos definir os seguintes subconjuntos de $R$ :

$$
R^{0}=\{\alpha \in R \mid(\alpha, \alpha)=0\}
$$

e

$$
R^{\times}=\{\alpha \in R \mid(\alpha, \alpha) \neq 0\} .
$$

Os elementos de $R^{0}$ e $R^{\times}$são chamados de raízes isotrópicas e raízes não-isotrópicas, respectivamente e $R=R^{\times} \cup R^{0}$.

(AE3) Se $\alpha \in R^{\times}$e $x_{\alpha} \in \mathcal{L}_{\alpha}$ então $a d_{\mathcal{L}} x_{\alpha}$ age localmente de forma nilpotente sobre $\mathcal{L}$, isto é, para cada $y \in \mathcal{L}$ existe um inteiro $n \geq 0$ tal que

$$
\left(a d x_{\alpha}\right)^{n} y=0
$$

Teorema 1.1.1. ([2], I, 1.29) Seja $\mathcal{L}$ uma álgebra de Lie safisfazendo (AE1), (AE2) e (AE3) e seja $\alpha \in R^{\times}$.

(a) Se $\beta \in R$ então $2 \frac{(\beta, \alpha)}{(\alpha, \alpha)} \in \mathbb{Z}$.

(b) Se $k \in \mathbb{C}$ e $k \alpha \in R$ então $k=-1,0,1$. 
(c) $\operatorname{dim}_{\mathbb{C}} \mathcal{L}_{\alpha}=1$

(d) Para cada $\beta \in R$ existem dois inteiros não-negativos u e d tais que, para cada $n \in \mathbb{Z}$ temos $\beta+n \alpha \in R$ se, e somente se, $-d \leq n \leq u$. Além disso, $d-u=2 \frac{(\beta, \alpha)}{(\alpha, \alpha)}$.

As duas outras propriedades que uma álgebra $\mathcal{L}$ possui dizem respeito ao seu sistema de raízes.

(AE4) $R$ é um subconjunto discreto em $\mathcal{H}^{*}$.

$($ AE5) $\mathcal{L}$ é irredutível no sentido que satisfaz:

(a) $R^{\times}$não se decompõe numa união disjunta $R_{1} \uplus R_{2}$, onde $R_{1}$ e $R_{2}$ são subconjuntos não-vazios de $R^{\times}$satisfazendo $\left(R_{1}, R_{2}\right)=\{0\}$.

(b) Para cada $\sigma \in R^{0}$ existe um $\alpha \in R^{\times}$tal que $\alpha+\sigma \in R$.

Observação 1.1.2. O propriedade (AE4) significa que a topologia relativa sobre $R$ é a topologia discreta ([2], I, 1.32).

Definição 1.1.3. Uma álgebra de Lie complexa satisfazendo as condições (AE1)-(AE5) chama-se álgebra de Lie afim estendida (ALAE) .

Exemplos 1.1.4. (1) Álgebras de Lie complexas simples de dimensão finita.

(2) Álgebras de Kac-Moody afins.

(3) Álgebras de Lie toroidais.

(4) Seja $q \in M_{n}(\mathbb{C})$ uma matriz $n \times n, q=\left(q_{i j}\right), 1 \leq i, j \leq n$, tal que $q_{i i}=1$ com $q_{i j}=q_{j i}^{-1}$. Então q chama-se matriz quântica. Seja $\mathbb{C}_{q}$ a álgebra gerada por $t_{i}^{ \pm 1}, 1 \leq i \leq n$ e o produto $t_{i} t_{j}$ definido por $t_{i} t_{j}=q_{i j} t_{j} t_{i}$ para todo $i, j$ com $1 \leq i, j \leq n$. Então $\mathbb{C}_{q}$ chama-se torus quântico deteminado pela matriz quântica q. Para todo $k, l \in \mathbb{Z}$ temos,

$$
t_{i}^{k} t_{j}^{l}=q_{i j}^{k l} t_{j}^{l} t_{i}^{k}
$$

A álgebra de Lie afim estendida mais natural é a extensão central da álgebra

$$
s l_{2}\left(\mathbb{C}_{q}\right)=\left\{\left(\begin{array}{cc}
x & y \\
z & w
\end{array}\right) \mid x, y, z, w \in \mathbb{C}_{q}, x+w \in\left[\mathbb{C}_{q}, \mathbb{C}_{q}\right]\right\},
$$

coordenada pelo torus quântico $\mathbb{C}_{q}$, onde $\left[\mathbb{C}_{q}, \mathbb{C}_{q}\right]=\left\{x y-y x \mid x, y \in \mathbb{C}_{q}\right\}$. 
Proposição 1.1.5. ([2], I, 2.1) Seja $\mathcal{L}$ um ALAE com sistema de raízes $R$. Seja $\delta \in R^{0}$ e $\alpha \in R$. Então $(\alpha, \delta)=0\left(\left(R^{0}, R\right)=0\right)$.

Observação 1.1.6. Uma forma bilinear, simétrica, invariante e não-degenerada sobre $\mathcal{L}$ multiplicada por um número complexo ainda é uma forma com tais propriedades. Portanto, podemos assumir que existe uma raiz não-isotrópica $\alpha \in R^{\times} \operatorname{com}(\alpha, \alpha) \in \mathbb{R}$. Daí se $\beta \in R^{\times}$temos $2 \frac{(\alpha, \beta)}{(\alpha, \alpha)} \in \mathbb{Z}$, então $(\alpha, \beta) \in \mathbb{R}$. Como $2 \frac{(\alpha, \beta)}{(\beta, \beta)} \in \mathbb{Z} \operatorname{temos}(\beta, \beta) \in \mathbb{R}$ se $(\alpha, \beta) \neq 0$. Devido ao item (a) da definição de irredutibilidade segue que $(\alpha, \beta) \in \mathbb{R}$, para todos $\alpha, \beta \in R$.

Definição 1.1.7. Seja $\mathcal{V}$ a expansão real das raízes de $R$.

Pela Observação 1.1.6 temos uma forma bilinear simétrica

$$
(\cdot, \cdot): \mathcal{V} \times \mathcal{V} \longrightarrow \mathbb{R}
$$

e podemos assumir que existe pelo menos um $\alpha \in R^{\times} \operatorname{com}(\alpha, \alpha)>0$.

Lema 1.1.8. ([2], I, 2.3) Seja $\beta \in R^{\times}$. Então $(\beta, \beta)>0$.

Seja $\mathcal{V}^{0}=\{\alpha \in \mathcal{V} \mid(\alpha, \beta)=0$, para todo $\beta \in \mathcal{V}\}$ o radical da forma sobre $\mathcal{V}$ e seja $\overline{\mathcal{V}}=\mathcal{V} / \mathcal{V}^{0}$. Como $R^{0} \subset \mathcal{V}^{0}$ e $R^{\times} \neq \emptyset$ temos $\mathcal{V} \neq \mathcal{V}^{0}$ então $\overline{\mathcal{V}} \neq\{0\}$ e, pela Proposição (1.1.5), segue que $R^{0}=R \cap \mathcal{V}^{0}$.

Seja $^{-}: \mathcal{V} \longrightarrow \overline{\mathcal{V}}$ a projeção canônica. A forma $(\cdot, \cdot)$ sobre $\mathcal{V}$ induz uma única forma bilinear, simétrica e não-degenerada $(\cdot, \cdot)$ sobre $\overline{\mathcal{V}}$. Então,

$$
(\bar{\alpha}, \bar{\beta})=(\alpha, \beta), \text { para todos } \alpha, \beta \in \mathcal{V} .
$$

Seja

$$
\bar{R}=\{\bar{\alpha} \mid \alpha \in R\}
$$

Como $R^{0}=R \cap \mathcal{V}^{0}$ temos

$$
\bar{R} \backslash\{0\}=\left\{\bar{\alpha} \mid \alpha \in R^{\times}\right\} .
$$

Lema 1.1.9. ([2], I, 2.10) $\bar{R}$ é finito. 
Lema 1.1.10. ([2], $I, 2.11) \bar{R} \backslash\{0\}$ não se decompõe como união disjunta $\bar{R}_{1} \uplus \bar{R}_{2}$, onde $\bar{R}_{1}$ e $\bar{R}_{2}$ são subconjuntos de $\bar{R} \backslash\{0\}$ satisfazendo $\left(\bar{R}_{1}, \bar{R}_{2}\right)=\{0\}$.

O seguinte teorema era a conjectura de Kac enunciada em [9] e provada em [2].

Teorema 1.1.11. ([2], I, 2.14) A forma $(\cdot, \cdot)$ sobre $\mathcal{V}$ é positivamente semi-definida.

Definição 1.1.12. Seja $\mathcal{V}$ um espaço vetorial real de dimensão finita com uma forma bilinear, simétrica e positivamente semi-definida e $R$ um subconjunto de $\mathcal{V}$. Seja

$$
R^{\times}=\{\alpha \in R \mid(\alpha, \alpha) \neq 0\} \text { e } R^{0}=\{\alpha \in R \mid(\alpha, \alpha)=0\} .
$$

$O$ subconjunto $R$ chama-se sistema de raízes afim estendido em $\mathcal{V}$ se $R$ satisfaz os seguintes axiomas:

(R1) $0 \in R$

(R2) $-R=R$

(R3) $R \operatorname{gera} \mathcal{V}$

(R4) $\alpha \in R^{\times}$implica que $2 \alpha \notin R$

(R5) $R$ é discreto em $\mathcal{V}$

(R6) Se $\alpha \in R^{\times}$e $\beta \in R$ então existem $d, u \in \mathbb{Z}_{\geq 0}$ tais que

$$
\{\beta+n \alpha \mid n \in \mathbb{Z}\} \cap\{\beta-d \alpha, \cdots, \beta+u \alpha\} \text { ed }-u=2 \frac{(\alpha, \beta)}{(\alpha, \alpha)}
$$

(R7) $R^{\times}$é irredutível.

Teorema 1.1.13. ([2], I, 2.16) Seja $\mathcal{L}$ uma $A L A E$ com sistema de raízes $R$. Então $R$ é um sistema de raízes afim estendido em $\mathcal{V}$.

Definição 1.1.14. Seja $\mathcal{X}$ um espaço vetorial real não-trivial de dimensão finita com uma forma bilinear, simétrica e positivamente definida $(\cdot, \cdot)$. Um subconjunto $\Delta$ de $\mathcal{X}$ chama-se sistema de raízes finito em $\mathcal{X}$ se

(a) $\Delta$ é finito, $0 \in \Delta$ e $\Delta$ gera $\mathcal{X}$. 
(b) Para cada $\alpha \in \Delta \backslash\{0\}$, a aplicação dada por $\beta \mapsto \beta-2 \frac{(\beta, \alpha)}{(\alpha, \alpha)} \alpha$, para todo $\beta \in \mathcal{X}$, estabiliza $\Delta$.

(c) Para cada $\alpha \in \Delta \backslash\{0\}, \beta \in \Delta$ temos $2 \frac{(\beta, \alpha)}{(\alpha, \alpha)} \in \mathbb{Z}$.

(d) $\Delta$ não se decompõe em uma união disjunta $\Delta_{1} \uplus \Delta_{2}$, onde $\Delta_{1}$ e $\Delta_{2}$ são subconjuntos não vazios de $\Delta$ satisfazendo $\left(\Delta_{1}, \Delta_{2}\right)=\{0\}$.

Teorema 1.1.15. ([2], I, 2.19) $\bar{R}$ é um sistema de raízes finito em $\overline{\mathcal{V}}$.

Como conseqüência do teorema acima, $\bar{R}$ pode ser de um dos tipos $A_{l}, B_{l}, C_{l}, D_{l}, E_{6}$, $E_{7}, E_{8}, F_{4}$ ou $G_{2}$. Assim, como em [2] e [21]:

Definição 1.1.16. $O$ tipo de $R$ ou de $\mathcal{L}$ é o tipo de $\bar{R}$.

\subsubsection{Grupo de Weyl afim estendido}

Seja $R$ um sistema de raízes afim estendido do tipo $A_{l}, D_{l}$ ou $E_{l}$. O grupo de Weyl afim estendido pode ser definido para os demais tipos de ALAE, porém como estamos interessados nas ALAE do tipo $A_{1}$ consideramos aqui somente as de conexão simples. Fixamos um sistema fundamental $\bar{\Pi}=\left\{\overline{\alpha_{1}}, \cdots, \overline{\alpha_{l}}\right\}$ de $\bar{R}$. Fixamos uma pré-imagem $\dot{\alpha}_{i} \in R$ de $\overline{\alpha_{i}}$. Seja $\dot{\mathcal{V}}$ a expansão real de $\dot{\alpha}_{1}, \cdots, \dot{\alpha}_{l}$. Então $\mathcal{V}=\dot{\mathcal{V}} \oplus \mathcal{V}^{0}$.

Consideremos o espaço vetorial real $\tilde{\mathcal{V}}=\dot{\mathcal{V}} \oplus \mathcal{V}^{0} \oplus\left(\mathcal{V}^{0}\right)^{*}$. Definimos a forma $(\cdot, \cdot)$ sobre $\tilde{\mathcal{V}}$ da seguinte forma:

$$
\begin{aligned}
\left(\dot{\mathcal{V}},\left(\mathcal{V}^{0}\right)^{*}\right) & =\{0\} \\
\left(\left(\mathcal{V}^{0}\right)^{*},\left(\mathcal{V}^{0}\right)^{*}\right) & =\{0\} \\
\left(\mathcal{V}^{0},\left(\mathcal{V}^{0}\right)^{*}\right) & \text { paridade natural. }
\end{aligned}
$$

Para $\alpha \in \tilde{\mathcal{V}} \operatorname{com}(\alpha, \alpha) \neq 0$ definimos a reflexão $r_{\alpha} \in G L(\tilde{\mathcal{V}})$ por

$$
r_{\alpha}(\lambda)=\lambda-2 \frac{(\lambda, \alpha)}{(\alpha, \alpha)} \alpha
$$

Definição 1.1.17. ([1]) $O$ grupo de Weyl afim estendido $\mathcal{W}_{R}$ de $R$ é definido como sendo o subgrupo de $G L(\tilde{\mathcal{V}})$ gerado pelas reflexões $r_{\alpha}$ para $\alpha \in R^{\times}$. 


\section{Capítulo 2}

\section{Álgebras TKK afins estendidas}

Seja $S$ um semi-reticulado de $\mathbb{R}^{\nu}, J(S)$ a álgebra de Jordan obtida a partir de $S$ e $\mathcal{G}(J(S))$ a álgebra de Lie obtida de $J(S)$ através da construção de Tits-Kantor-Koecher. Uma álgebra de Lie afim estendida obtida a partir de um semi-reticulado foi introduzida em [2] (p. 79) como exemplo de uma álgebra de Lie afim estendida do tipo $A_{1}$. Em [19], o autor estuda a representação através de operadores de vértice da extensão central universal $\widehat{\mathcal{G ( J )}}$ de $\mathcal{G}(J)$ quando $S$ é um semi-reticulado (não reticulado) do $\mathbb{R}^{2}$ e denomina tal álgebra de baby TKK por se tratar da álgebra de Lie com menor o sistema de raízes afim estendido [18] que não é do tipo finito e nem do tipo afim. A álgebra TKK afim estendida é um quociente da álgebra $\widehat{\mathcal{G}(J)}$ quando ela é estendida por derivações do grau e por esse motivo denominamos de tal forma. Nessa seção daremos as definições e conceitos básicos para introduzir as álgebras TKK afins estendidas e seu sistema de raízes.

\subsection{Semi-reticulados do $\mathbb{R}^{\nu}$}

Denotamos por $\mathcal{U}$ um $\mathbb{R}$-espaço vetorial de dimensão finita $\nu$.

Definição 2.1.1. Um subconjunto $S \subset \mathcal{U}$ chama-se semi-reticulado $\mathrm{em} \mathcal{U}$, se $S$ satisfaz as seguintes condições:

(S1) $0 \in S$

(S2) $-S=S$ 
(S3) $S+2 S \subseteq S$

(S4) $S$ gera $\mathcal{U}$

(S5) $S$ é discreto em $\mathcal{U}$.

Definição 2.1.2. Na definição anterior se substituírmos a condição $(S 3)$ por $S+S \subseteq S$ então $S$ será um reticulado.

Denotamos por $\langle X\rangle$ o subgrupo aditivo gerado por um subconjunto $X$ de $\mathcal{U}$.

Proposição 2.1.3. ([2], II, 1.4) Seja $S$ um subconjunto de $\mathcal{U}$. Se $S$ é um semi-reticulado em $\mathcal{U}$ e $\Lambda=\langle S\rangle$, então $\Lambda$ é um reticulado em $\mathcal{U}$ tal que

$$
2 \Lambda \subseteq S \subseteq \Lambda \text { e } 2 \Lambda+S \subseteq S
$$

Reciprocamente, se existe um reticulado $\Lambda$ em $\mathcal{U}$ tal que a condição (2.1) é satisfeita, então $S$ é um semi-reticulado em $\mathcal{U}$.

A condição (2.1) implica que podemos considerar $S$ como união de conjuntos de classes laterais de $2 \mathbb{Z}^{\nu}$ em $\mathbb{Z}^{\nu}$, incluindo a classe lateral $2 \mathbb{Z}^{\nu}$, isto é,

$$
S=\cup_{i=0}^{m} S_{i}
$$

onde $S_{i}$ são classes laterais distintas de $2 \mathbb{Z}^{\nu}$ em $\mathbb{Z}^{\nu}$ e $S_{0}=2 \mathbb{Z}^{\nu}$.

Para $\sigma \in S$ seja $x^{\sigma}$ um símbolo e definimos o seguinte $\mathbb{C}$-espaço vetorial:

$$
J=J(S)=\bigoplus_{\sigma \in S} \mathbb{C} x^{\sigma}
$$

com a multiplicação dada por

$$
x^{\sigma} x^{\tau}= \begin{cases}x^{\sigma+\tau} & , \text { se } \sigma, \tau \in S_{0} \cup S_{i}, \quad 0 \leq i \leq m, \\ 0 & , \text { caso contrário. }\end{cases}
$$

Então $J$ é uma álgebra comutativa com elemento identidade $1=x^{0}$.

Proposição 2.1.4. ([2], III, 2.1) $J(S)$ é uma álgebra de Jordan. 
Consideremos a construção de Tits-Kantor-Koecher ([11], p. 324). Através dela obtemos uma álgebra de Lie a partir de qualquer álgebra de Jordan com unidade. No nosso caso consideramos a álgebra de Jordan acima mencionada. O processo inicia-se com a construção da álgebra de Lie de transformações lineares em $J$, gerada pelas multiplicações à esquerda por elementos de $J, L_{a}$ para $a \in J$. Denotemos essa álgebra de Lie por $L_{J}$. Consideramos a álgebra de Lie de derivações internas de $J$,

$$
\operatorname{Inder}(J)=\left\{\sum_{i}\left[L_{b_{i}}, L_{c_{i}}\right] \mid b_{i}, c_{i} \in J\right\}
$$

e a álgebra de Lie conhecida como álgebra de Lie de estrutura interna de J:

$$
\operatorname{Instrl}(J)=L_{J} \oplus \operatorname{Inder}(J),
$$

com o produto definido por

$$
\left[L_{a}+D, L_{b}+E\right]=\left[L_{a}, L_{b}\right]+L_{D b}-L_{E a}+[D, E],
$$

para $a, b \in J$ e $D, E \in \operatorname{Inder}(J)$. A álgebra de Lie $\operatorname{Instrl}(J)$ possui um automorfismo de ordem dois dado por $\overline{\left[L_{a}+D\right]}=-L_{a}+D$.

Seja $\bar{J}$ uma cópia linear da álgebra de Jordan $J$. Então

$$
\mathcal{G}(J)=J \oplus \operatorname{Instrl}(J) \oplus \bar{J}
$$

é uma álgebra de Lie sob o produto

$$
\left[a_{1}+\bar{b}_{1}+E_{1}, a_{2}+\bar{b}_{2}+E_{2}\right]=-E_{2} a_{1}+E_{1} a_{2}-\overline{E_{2} b_{1}}+\overline{E_{1} b_{2}}+a_{1} \Delta b_{2}-a_{2} \Delta b_{1}+\left[E_{1}, E_{2}\right]
$$

para $a_{i} \in J, b_{i} \in \bar{J}$ e $E_{i} \in \operatorname{Instrl}(J)$, onde $a \Delta b=L_{a b}+\left[L_{a}, L_{b}\right]$.

Para facilitar as manipulações algébricas idenficamos a álgebra de Lie $\mathcal{G}(J)$ com a seguinte álgebra de Lie.

Seja $e_{+}, e_{-}$e $h$ a base standard de Chevalley de $s l_{2}(\mathbb{C})$. Definimos o espaço vetorial

$$
\mathcal{G}^{\prime}(J)=\left(\operatorname{sl}_{2}(\mathbb{C}) \otimes J\right) \oplus \operatorname{Inder}(J)
$$


com o produto

$$
\begin{aligned}
{[A \otimes a, B \otimes b] } & =[A, B] \otimes a b+(A, B)\left[L_{a}, L_{b}\right], \\
{[D, A \otimes a] } & =A \otimes D a \\
{\left[D,\left[L_{a}, L_{b}\right]\right] } & =\left[L_{D a}, L_{b}\right]+\left[L_{a}, L_{D b}\right] .
\end{aligned}
$$

para $A, B \in \operatorname{sl}_{2}(\mathbb{C}), a, b \in J$ e $D \in \operatorname{Inder}(J)$, onde $(A, B)=2 \operatorname{tr}(A, B)$. O espaço vetorial $\mathcal{G}^{\prime}(J)$ munido com o produto acima é uma álgebra de Lie. Para verificar a identidade Jacobi precisamos dos seguintes lemas, os quais são bem conhecidos:

Lema 2.1.5. Sejam $a_{i} \in J, i=1,2,3$. Então

$$
\begin{aligned}
{\left[L_{a_{1}}, L_{a_{2}}\right]+\left[L_{a_{2}}, L_{a_{1}}\right] } & =0 \\
{\left[L_{a_{1} a_{2}}, L_{a_{3}}\right]+\left[L_{a_{2} a_{3}}, L_{a_{1}}\right]+\left[L_{a_{3} a_{1}}, L_{a_{2}}\right] } & =0
\end{aligned}
$$

Lema 2.1.6. Sejam $A, B, C \in \operatorname{sl}_{2}(\mathcal{C})$. Então

$$
[[A, B], C]=(B, C) A-(A, C) B
$$

onde $(A, B)=2 \operatorname{tr}(A B)$.

Vamos verificar que $\mathcal{G}^{\prime}(J)$ com o produto acima definido é uma álgebra de Lie. A anticomutatividade é de simples verificação, portanto vamos checar a identidade de Jacobi. Denotamos por $[x, y, z]:=[x,[y, z]]+[y,[z, x]]+[z,[x, y]]$, para $x, y, z \in \mathcal{G}^{\prime}(J)$, então vamos provar que

$$
\begin{aligned}
{[A \otimes a, B \otimes b, C \otimes c] } & =0, \\
{[A \otimes a, B \otimes b, D] } & =0, \\
{\left[A \otimes a, D_{1}, D_{2}\right] } & =0, \\
{\left[D_{1}, D_{2}, D_{3}\right] } & =0,
\end{aligned}
$$

para $A, B, C \in \operatorname{sl}_{2}(\mathbb{C}), a, b, c \in J$ e $D, D_{1}, D_{2}, D_{3} \in \operatorname{Inder}(J)$.

A igualdade (2.6) se verifica pelo fato de $\operatorname{Inder}(J)$ ser uma álgebra de Lie. Então 
vamos verificar as igualdades (2.3), (2.4) e (2.5). Temos

$$
\begin{aligned}
{[A \otimes a,[B \otimes b, C \otimes c]] } & =[A,[B, C]] \otimes a(b c)+(A,[B, C])\left[L_{a}, L_{b c}\right]-(B, C) A \otimes b(c a) \\
+ & (B, C) A \otimes c(b a) \\
{[B \otimes b,[C \otimes c, A \otimes a]] } & =[B,[C, A]] \otimes b(c a)+(B,[C, A])\left[L_{b}, L_{c a}\right]-(C, A) B \otimes c(a b) \\
+ & (C, A) B \otimes a(c b) \\
{[C \otimes c,[A \otimes a, B \otimes b]] } & =[C,[A, B]] \otimes c(a b)+(C,[A, B])\left[L_{c}, L_{a b}\right]-(A, B) C \otimes a(b c) \\
+ & (A, B) C \otimes b(a c)
\end{aligned}
$$

Daí,

$$
\begin{aligned}
{[A \otimes a, B \otimes b, C \otimes c] } & =\{[A,[B, C]]-(A, B) C+(C, A) B\} \otimes a(b c) \\
& +\{[B,[C, A]]-(B, C) A+(A, B) C\} \otimes b(c a) \\
& +\{[C,[A, B]]-(C, A) B+(B, C) A\} \otimes c(a b) \\
& +(A,[B, C])\left(\left[L_{a}, L_{b c}\right]+\left[L_{b}, L_{c a}\right]+\left[L_{c}, L_{a b}\right]\right)
\end{aligned}
$$

Pelos Lemas 2.1.5 e 2.1.6 segue que $[A \otimes a, B \otimes b, C \otimes c]=0$. Agora

$$
\begin{aligned}
& {[A \otimes a,[B \otimes b, D]]=-[A, B] \otimes a D b-(A, B)\left[L_{a}, L_{D b}\right]} \\
& {[B \otimes b,[D, A \otimes a]]=-[A, B] \otimes b D a+(A, B)\left[L_{b}, L_{D a}\right]} \\
& {[D,[A \otimes a, B \otimes b]]=[A, B] \otimes D(a b)+(A, B)\left[L_{D a}, L_{b}\right]+(A, B)\left[L_{a}, L_{D b}\right]}
\end{aligned}
$$

Logo, $[A \otimes a, B \otimes b, D]=0$. Finalmente,

$$
\begin{aligned}
& {\left[A \otimes a,\left[D_{1}, D_{2}\right]\right]=-A \otimes D_{1} D_{2} a+A \otimes D_{2} D_{1} a} \\
& {\left[D_{1},\left[D_{2}, A \otimes a\right]\right]=-A \otimes D_{2} D_{1} a} \\
& {\left[D_{2},\left[D_{1}, A \otimes a\right]\right]=A \otimes D_{1} D_{2} a}
\end{aligned}
$$

Potanto, $\left[A \otimes a, D_{1}, D_{2}\right]=0$.

Proposição 2.1.7. ([19], 1.10) $\mathcal{G}^{\prime}(J)$ é isomorfa à álgebra de Lie $\mathcal{G}(J)$ via o homomorfismo $\varphi: \mathcal{G}^{\prime}(J) \longrightarrow \mathcal{G}(J)$ definido por

$$
\begin{aligned}
e_{+} \otimes a & \mapsto \sqrt{2} a, \\
e_{-} \otimes \bar{a} & \mapsto \sqrt{2} \bar{a}, \\
h \otimes a & \mapsto 2 L_{a}, \\
{\left[L_{a}, L_{b}\right] } & \mapsto\left[L_{a}, L_{b}\right] .
\end{aligned}
$$


A partir daqui identificamos $\mathcal{G}(J)$ com a álgebra de Lie $\mathcal{G}^{\prime}(J)$.

Seja $\epsilon: J \longrightarrow \mathbb{C}$ uma aplicação $\mathbb{C}$-linear definida pela expansão linear de

$$
\epsilon\left(x^{\sigma}\right)= \begin{cases}1 & , \text { se } \sigma=0 \\ 0 & , \text { se } \sigma \neq 0\end{cases}
$$

Então a aplicação $(\cdot, \cdot): J \times J \longrightarrow \mathbb{C}$ dada por $(a, b)=\epsilon(a b)$ é uma forma bilinear, simétrica, associativa e não-degenerada sobre $J$ e ela determina em $\mathcal{G}(J)$ uma única forma bilinear, simétrica, invariante e não-degenerada ([2], p. 81) definida por

$$
\begin{aligned}
(A \otimes a, B \otimes b) & =(A, B) \epsilon(a b), \\
(A \otimes a, D) & =0, \\
\left(D,\left[L_{a}, L_{b}\right]\right) & =\epsilon((D a) b),
\end{aligned}
$$

onde $A, B \in \operatorname{sl}_{2}(\mathbb{C}), a, b \in J, D \in \operatorname{Inder}(J)$.

Seja $\dot{\mathcal{H}}:=\mathbb{C} h \otimes x^{0}=\mathbb{C} h \otimes 1$ a subálgebra abeliana de $\mathcal{G}(J)$. Em relação à ação adjunta de $\dot{\mathcal{H}}, \mathcal{G}(J)$ decompõe-se em

$$
\mathcal{G}(J)=\mathcal{G}_{\dot{\alpha}}(J) \oplus \mathcal{G}_{0}(J) \oplus \mathcal{G}_{-\dot{\alpha}}(J)
$$

onde $\dot{\alpha} \in \dot{\mathcal{H}}^{*}$ satisfaz $\dot{\alpha}(h \otimes 1)=1 \mathrm{e}$

$$
\mathcal{G}_{ \pm \dot{\alpha}}(J)=\mathbb{C} e_{ \pm} \otimes J \text { e } \mathcal{G}_{0}(J)=\operatorname{Inder}(J)+\mathbb{C} h \otimes J .
$$

A álgebra de Jordan $J(S)$ é uma álgebra $\mathbb{Z}^{\nu}$-graduada com

$$
J^{\sigma}= \begin{cases}\mathbb{C} x^{\sigma} & , \text { se } \sigma \in S \\ \{0\} & , \text { se } \sigma \notin S .\end{cases}
$$

Essa graduação induz uma $\mathbb{Z}^{\nu}$-graduação sobre $\mathcal{G}(J)$ dada por

$$
\mathcal{G}(J)=\oplus_{\sigma \in \mathbb{Z}^{\nu}} \mathcal{G}^{\sigma}(J),
$$

onde $\mathcal{G}^{\sigma}(J)=\mathcal{G}_{+}^{\sigma}(J)+\mathcal{G}_{0}^{\sigma}(J)+\mathcal{G}_{-}^{\sigma}(J)$, com

$$
\mathcal{G}_{ \pm}^{\sigma}(J)= \begin{cases}\mathbb{C} e_{ \pm} \otimes x^{\sigma} & , \text { se } \sigma \in S \\ \{0\} & , \text { se } \sigma \notin S\end{cases}
$$

e

$$
\mathcal{G}_{0}^{\sigma}(J)=\mathbb{C} h \otimes x^{\sigma}+\sum_{\tau, \xi \in \mathbb{Z}^{\nu}, \tau+\xi=\sigma} \mathbb{C}\left[L_{x^{\tau}}, L_{x}\right], \text { para } \sigma \in \mathbb{Z}^{\nu}
$$


Sejam $\mathbf{d}_{\mathbf{i}} \in \operatorname{Der}(\mathcal{G}(J))$, para $i=1, \cdots, \nu$, definidas por $\mathbf{d}_{\mathbf{i}} x=n_{i} x$, para $x \in \mathcal{G}^{\sigma}(J)$ e $\sigma=\left(n_{1}, \cdots, n_{\nu}\right)$.

Construímos uma álgebra de Lie afim estendida a partir de $\mathcal{G}(J)$ definindo o seguinte espaço vetorial

$$
\mathcal{L}(J)=\mathcal{G}(J) \oplus \mathcal{C} \oplus \mathcal{D}
$$

onde $\mathcal{C}=\mathbb{C c}_{\mathbf{1}} \oplus \cdots \oplus \mathbb{C c}_{\nu}$ é um espaço vetorial de dimensão $\nu$ e $\mathcal{D}=\mathbb{C d}_{\mathbf{1}} \oplus \cdots \oplus \mathbb{C d}_{\nu} \subset$ $\operatorname{Der}(\mathcal{G}(J))$ e o seguinte produto em $\mathcal{L}(J)$

$$
\begin{aligned}
{[\mathcal{L}(J), \mathcal{C}] } & =[\mathcal{D}, \mathcal{D}]=\{0\} \\
{\left[\mathbf{d}_{\mathbf{i}}, x\right] } & =\mathbf{d}_{\mathbf{i}} x, \text { para } x \in \mathcal{G}(J), \\
{[A \otimes a, B \otimes b] } & =[A, B] \otimes a b+(A, B)\left[L_{a}, L_{b}\right]+\sum_{i=1}^{\nu}\left(d_{i}(A \otimes a), B \otimes b\right) c_{i}, \\
{[D, A \otimes a] } & =A \otimes D a \\
{\left[D,\left[L_{a}, L_{b}\right]\right] } & =\left[L_{D a}, L_{b}\right]+\left[L_{a}, L_{D b}\right]+\sum_{i=1}^{\nu}\left(\mathbf{d}_{\mathbf{i}} D,\left[L_{a}, L_{b}\right]\right) \mathbf{c}_{\mathbf{i}} .
\end{aligned}
$$

Chamamos a álgebra de Lie $\mathcal{L}(J)$ de álgebra TKK afim estendida.

Estendemos a forma $(\cdot, \cdot)$ de $\mathcal{G}(J)$ sobre $\mathcal{L}(J)$ definindo

$$
\begin{aligned}
(\mathcal{C}, \mathcal{C}) & =(\mathcal{D}, \mathcal{D})=\{0\} \\
\left(\mathbf{c}_{\mathbf{i}}, \mathbf{d}_{\mathbf{j}}\right) & =\delta_{i j}, \text { para } i, j=1, \cdots, \nu, \\
(\mathcal{C}, \mathcal{G}(J)) & =(\mathcal{D}, \mathcal{G}(J))=\{0\} .
\end{aligned}
$$

A forma $(\cdot, \cdot)$ estendida sobre $\mathcal{L}(J)$ é não-degenerada e invariante ([2],III, 1.14).

Teorema 2.1.8. ([2], III, 2.9) $\mathcal{L}(J)$ é uma álgebra de Lie afim estendida do tipo $A_{1}$.

Sejam $\mathcal{H}=\dot{\mathcal{H}} \oplus \mathcal{C} \oplus \mathcal{D}$ e $\mathcal{H}^{*}=\dot{\mathcal{H}}^{*} \oplus \mathcal{C}^{*} \oplus \mathcal{D}^{*}$. Seja $\left\{\sigma_{1}, \cdots, \sigma_{\nu}\right\}$ uma base de $\mathcal{D}^{*}$ dual à $\left\{\mathbf{d}_{\mathbf{1}}, \cdots, \mathbf{d}_{\nu}\right\}$. Então identificamos $\mathbb{Z}^{\nu} \subseteq \mathcal{D}^{*}$ por

$$
\left(n_{1}, \cdots, n_{\nu}\right)=\sum_{i=1}^{\nu} n_{i} \sigma_{i}
$$

e escrevemos

$$
[d, x]=\sigma(d) x, \text { para } d \in \mathcal{D}, x \in \mathcal{G}^{\sigma}(J), \sigma \in \mathbb{Z}^{\nu}
$$


Para $\alpha \in \mathcal{H}^{*}$ seja

$$
\mathcal{L}_{\alpha}(J)=\{x \in \mathcal{L}(J) \mid[h, x]=\alpha(h) x, \text { para todo } h \in \mathcal{H}\}
$$

Então podemos decompor $\mathcal{L}(J)$ em espaços de raízes,

$$
\mathcal{L}(J)=\sum_{\alpha \in \mathcal{H}^{*}} \mathcal{L}_{\alpha}(J)=\sum_{\sigma \in \mathbb{Z}^{\nu}} \sum_{\dot{\alpha} \in \dot{R}} \mathcal{L}_{\dot{\alpha}+\sigma}(J),
$$

onde $\mathcal{L}_{0}(J)=\mathcal{G}_{0}(J) \cap \mathcal{G}^{0}(J) \oplus \mathcal{C} \oplus \mathcal{D}=\dot{\mathcal{H}} \oplus \mathcal{C} \oplus \mathcal{D}$ e $\mathcal{L}_{\dot{\alpha}+\sigma}(J)=\mathcal{G}_{\dot{\alpha}}(J) \cap \mathcal{G}^{\sigma}(J)$, para $\dot{\alpha}, \sigma$ não ambos nulos.

Denotmos por $\dot{R}=\left\{\dot{\alpha} \in \dot{\mathcal{H}} \mid \mathcal{G}_{\dot{\alpha}}(J) \neq\{0\}\right\}$. Seja $R=\left\{\alpha \in \mathcal{H}^{*} \mid \mathcal{L}_{\alpha}(J) \neq\{0\}\right\}=$ $\left\{\dot{\alpha}+\sigma \mid \dot{\alpha} \in \dot{R}, \sigma \in \mathbb{Z}^{\nu}\right.$ e $\left.\mathcal{G}_{\dot{\alpha}}(J) \cap \mathcal{G}^{\sigma}(J) \neq\{0\}\right\}$ o sistema de raízes de $\mathcal{L}(J)$ que é do tipo $A_{1}$. Pela construção 3.2 de [2] (página 34) o sistema de raízes afim estendida de $\mathcal{L}(J)$ é da seguinte forma

$$
R=(S+S) \cup(\dot{R}+S)
$$

Consideramos o seguinte sistema de raízes afim estendido

$$
\widetilde{R}=\langle S\rangle \cup(\dot{R}+\langle S\rangle)
$$

Observamos que $R \subseteq \widetilde{R}$.

Sejam $\left\{\delta_{1}, \cdots, \delta_{\nu}\right\}$ uma base de $\mathcal{V}^{0}$ e $\Lambda_{1}, \cdots, \Lambda_{\nu}$ uma base de $\left(\mathcal{V}^{0}\right)^{*}$ dual a $\left\{\delta_{1}, \cdots, \delta_{\nu}\right\}$. Então $\tilde{\mathcal{V}}=\mathbb{R} \dot{\alpha} \oplus \sum_{i=1}^{\nu} \mathbb{R} \delta_{i} \oplus \sum_{i=1}^{\nu} \mathbb{R} \Lambda_{i}$.

Sejam

$$
\begin{aligned}
I & =\{1,2, \cdots, \nu\} \\
\dot{Q} & =\mathbb{Z} \dot{R} \\
Q & =\mathbb{Z} \tilde{R}=\dot{Q}+\sum_{i=1}^{\nu} \mathbb{Z} \delta_{i}
\end{aligned}
$$

e

$$
\dot{\mathcal{W}}=\left\langle r_{\dot{\alpha}}\right\rangle \leq G L(\tilde{\mathcal{V}})
$$

Sejam $\alpha \in \mathcal{V}$ uma raiz não-isotrópica e $\delta \in \mathcal{V}^{0}$. Então, para $\lambda \in \tilde{\mathcal{V}}$, temos

$$
r_{\alpha+\delta} r_{\alpha}(\lambda)=\lambda-(\lambda, \delta) \alpha^{\vee}+\left[\left(\lambda, \alpha^{\vee}\right)-\frac{1}{2}\left(\alpha^{\vee}, \alpha^{\vee}\right)(\lambda, \delta)\right] \delta
$$


onde $\alpha^{\vee}=2 \frac{\alpha}{(\alpha, \alpha)}$. Normalizando a forma para $\alpha \in \widetilde{R}$, considerando $(\alpha, \alpha)=2$, temos

$$
r_{\alpha+\delta} r_{\alpha}(\lambda)=\lambda-(\lambda, \delta) \alpha+\left[(\lambda, \alpha)-\frac{1}{2}(\alpha, \alpha)(\lambda, \delta)\right] \delta
$$

Para $j \in I$ e $\alpha \in Q$ definimos uma aplicação linear $t_{\alpha}^{j}: \widetilde{\mathcal{V}} \longrightarrow \widetilde{\mathcal{V}}$ por

$$
t_{\alpha}^{j}(\lambda)=\lambda-\left(\lambda, \delta_{j}\right) \alpha+\left[(\lambda, \alpha)-\frac{1}{2}(\alpha, \alpha)\left(\lambda, \delta_{j}\right)\right] \delta_{j} .
$$

Para $\alpha \in \widetilde{R}$, temos $t_{\alpha}^{j}=r_{\alpha+\delta_{j}} r_{\alpha}$.

Denotamos por $\mathcal{W}$ e $\tilde{\mathcal{W}}$ os grupos de Weyl afins estendidos de $R$ e $\widetilde{R}$, respectivamente. Pela Proposiçao 2.18 de [1] temos que $\mathcal{W} \triangleleft \tilde{\mathcal{W}}$.

Consideramos os seguintes subgrupos de $\tilde{\mathcal{W}}$ e $\mathcal{W}$, respectivamente, dados por:

$$
\tilde{H}:=\left\langle r_{\alpha+\delta} r_{\alpha}: \alpha \in \tilde{R}^{\times}, \delta \in \mathcal{V}^{0}, \alpha+\delta \in \tilde{R}^{\times}\right\rangle \leq \tilde{\mathcal{W}}
$$

e

$$
H:=\left\langle r_{\alpha+\delta} r_{\alpha}: \alpha \in R^{\times}, \delta \in \mathcal{V}^{0}, \alpha+\delta \in R^{\times}\right\rangle \leq \mathcal{W} .
$$

Lema 2.1.9. ([1],3.12) $\tilde{H}=\left\langle t_{\alpha}^{j}:(j, \alpha) \in I \times Q\right\rangle$.

Proposição 2.1.10. ([1],3.27) $\tilde{\mathcal{W}}=\dot{\mathcal{W}} \ltimes \tilde{H}$ e $\mathcal{W}=\dot{\mathcal{W}} \ltimes H$

\subsection{Semi-Reticulado do $\mathbb{R}^{2}$}

Seja $\mathbb{R}^{2}=\mathbb{R} \delta_{1} \oplus \mathbb{R} \delta_{2}$, onde $\delta_{1}=(1,0)$ e $\delta_{2}=(0,1)$. Seja $S$ o seguinte semi-reticulado (não-reticulado) de $\mathbb{R}^{2}$ :

$$
S=S_{0} \cup S_{1} \cup S_{2}
$$

onde $S_{0}=2 \mathbb{Z} \delta_{1}+2 \mathbb{Z} \delta_{2}$ e $S_{i}=\delta_{i}+S_{0}, i=1,2$. Para $\sigma_{i}=a_{i} \delta_{1}+b_{i} \delta_{2}, \operatorname{com} a_{i}, b_{i} \in \mathbb{R}$ e $i=1,2$, denotamos por $\sigma_{1} \cdot \sigma_{2}=a_{1} a_{2}+b_{1} b_{2}$ o produto interno de $\sigma_{1}$ com $\sigma_{2}$. Seja $S^{\perp}:=\mathbb{Z}^{2} \backslash S$.

Lema 2.2.1. ([19],2.13) Sejam $\sigma, \tau \in S$. Denotamos por $v_{\rho}=\left[L_{x^{\delta_{1}}}, L_{x^{\rho-\delta_{1}}}\right]$, para $\rho \in S^{\perp}$. Então temos

$$
\left[L_{x^{\sigma}}, L_{x^{\tau}}\right]= \begin{cases}0 & , \text { se } \sigma+\tau \in S, \\ v_{\sigma+\tau} & , \text { se } \sigma+\tau \in S^{\perp} \text { e } \sigma \in S_{1}, \\ -v_{\sigma+\tau} & , \text { se } \sigma+\tau \in S^{\perp} \text { e } \sigma \in S_{2},\end{cases}
$$


$e\left\{v_{\rho}\right\}_{\rho \in S^{\perp}}$ forma uma base de $\operatorname{Inder}(J)$.

Lema 2.2.2. Para $S=S_{0} \cup S_{1} \cup S_{2}$ o semi-reticulado não-reticulado de $\mathbb{R}^{2}$ a subálgebra Inder $(J)$ de $\mathcal{G}(J)$ é uma subálgebra abeliana.

Prova: Consideramos a aplicação $\Omega: S \longrightarrow \mathbb{C}$ dada por $\Omega(\tau)=\frac{1}{2}\left((-1)^{\tau \cdot \delta_{1}}-(-1)^{\tau \cdot \delta_{2}}\right)$, para $\tau \in S$. Então $\left[L_{x^{\delta_{1}}}, L_{x^{\sigma-\delta_{1}}}\right] x^{\tau}=\Omega(\tau) x^{\sigma+\tau}$, para $\sigma \in S^{\perp}$ e $\tau \in S$.

Devido ao Lema 2.2.1, podemos considerar somente o produto de dois elementos arbitrários $v_{\sigma}$ e $v_{\tau} \in \operatorname{Inder}(J)$, para $\sigma, \tau \in S^{\perp}$. Pela relação (2.2) temos

$$
\begin{aligned}
{\left[v_{\sigma}, v_{\tau}\right] } & =\left[\left[L_{x^{\delta_{1}}}, L_{x^{\sigma-\delta_{1}}}\right],\left[L_{x^{\delta_{1}}}, L_{x^{\tau-\delta_{1}}}\right]\right] \\
& =\left[L_{\left[L_{x^{\delta_{1}}}, L_{x^{\sigma-\delta_{1}}}\right] x^{\delta_{1}}}, L_{x^{\tau-\delta_{1}}}\right]+\left[L_{x^{\delta_{1}}}, L_{\left[L_{x^{\delta_{1}}}, L_{x^{\sigma-\delta_{1}}}\right] x^{\tau-\delta_{1}}}\right] \\
& =\left[L_{\Omega\left(\delta_{1}\right) x^{\sigma+\delta_{1}}}, L_{x^{\tau-\delta_{1}}}\right]+\left[L_{x^{\delta_{1}}}, L_{\Omega\left(\tau-\delta_{1}\right) x^{\sigma+\tau-\delta_{1}}}\right]
\end{aligned}
$$

Como $\sigma+\delta_{1}+\tau-\delta_{1}=\sigma+\tau \in S$, pelo Lema 2.2.1, temos

$$
\left[L_{\Omega\left(\delta_{1}\right) x^{\sigma+\delta_{1}}}, L_{x^{\tau-\delta_{1}}}\right]=0, \quad\left[L_{x^{\delta_{1}}}, L_{\Omega\left(\tau-\delta_{1}\right) x^{\sigma+\tau-\delta_{1}}}\right]=0
$$

e, portanto, $\left[v_{\sigma}, v_{\tau}\right]=0$.

Devido ao Lema 2.2.1, a graduação em $\mathcal{G}(J)$ será da seguinte forma

$$
\mathcal{G}(J)=\bigoplus_{\sigma \in \mathbb{Z}^{2}} \mathcal{G}^{\sigma}(J)
$$

onde $\mathcal{G}^{\sigma}(J)=\mathcal{G}_{+}^{\sigma}(J)+\mathcal{G}_{0}^{\sigma}(J)+\mathcal{G}_{-}^{\sigma}(J)$, com

$$
\begin{gathered}
\mathcal{G}_{ \pm}^{\sigma}(J)= \begin{cases}\mathbb{C} e_{ \pm} \otimes x^{\sigma} & , \text { se } \sigma \in S, \\
\{0\} & , \text { se } \sigma \in S^{\perp},\end{cases} \\
\mathcal{G}_{0}^{\sigma}(J)= \begin{cases}\mathbb{C} h \otimes x^{\sigma} & , \text { se } \sigma \in S, \\
\mathbb{C}\left[L_{x^{\delta_{1}}}, L_{x^{\sigma-\delta_{1}}}\right] & , \text { se } \sigma \in S^{\perp} .\end{cases}
\end{gathered}
$$

Pelo Lema 2.2.2, a álgebra TKK afim estendida

$$
\mathcal{L}(J)=\mathcal{G}(J) \oplus \mathcal{C} \oplus \mathcal{D}
$$


onde $\mathcal{C}=\mathbb{C c}_{1} \oplus \mathbb{C c}_{\mathbf{2}}$ e $\mathcal{D}=\mathbb{C d}_{\mathbf{1}} \oplus \mathbb{C d}_{2}$, terá o produto

$$
\begin{gathered}
{[A \otimes a, B \otimes b]=[A, B] \otimes a b+(A, B)\left[L_{a}, L_{b}\right]+\sum_{i=1,2}\left(d_{i}(A \otimes a), B \otimes b\right) c_{i},} \\
{[D, A \otimes a]=A \otimes D a, \quad\left[D,\left[L_{a}, L_{b}\right]\right]=\sum_{i=1,2}\left(d_{i} D,\left[L_{a}, L_{b}\right]\right) c_{i},} \\
{[\mathcal{L}(J), \mathcal{C}]=\{0\}=[\mathcal{D}, \mathcal{D}], \quad\left[d_{i}, y\right]=d_{i} y, \quad i=1,2, \quad y \in \mathcal{G}(J),}
\end{gathered}
$$

onde $A, B \in \operatorname{sl}_{2}(\mathbb{C}), a, b \in J$ e $D \in \operatorname{Inder}(J)$.

O sistema de raízes de $\mathcal{L}(J)$ é $R=\mathbb{Z}^{2} \cup(\dot{R}+S)$, onde $\dot{R}=\{-\dot{\alpha}, \dot{\alpha}\}$. 


\section{Capítulo 3}

\section{Módulo de Verma Clássico}

A definição de sistema de raízes positivo foi introduzida por Jakobsen e Kac em [12], onde realizaram a classificação de todos os sistemas de raízes positivos, a menos de equivalência, de um sistema de raízes afim, feito independentemente por Futorny [7]. Seguindo essa linha classificamos os sistemas de raízes positivos, quando fixado o conjunto positivo de raízes isotrópicas para a álgebra TKK afim estendida da seção 2.2. Obtivemos quatro órbitas da subálgebra de Borel que levam a distintos módulos tipo Verma, sendo um deles uma generalização do módulo de Verma clássico para o caso afim e o outro uma generalização do módulo de Verma imaginário. Provamos que o módulo de Verma clássico sobre a álgebra TKK afim estendida é irredutível se, e somente se, o módulo de Verma sobre sua subálgebra, que é isomorfa à álgebra $A_{1}^{(1)}$, é irredutível.

\subsection{Sistema de raízes positivo}

Consideramos aqui a álgebra TKK afim estendida $\mathcal{L}(J)$ definida no Capítulo 2 e seu sistema de raízes $R$.

Definição 3.1.1. Um submonóide em $\Psi \subset R^{0}$ chama-se conjunto positivo de raízes isotrópicas se

(i) $\Psi \cup(-\Psi)=R^{0} e$

(ii) $\Psi \cap(-\Psi)=\{0\}$. 
Enunciaremos o seguinte resultado que aparece em [4] modificando a nome de raízes imaginárias por raízes isotrópicas. Denotamos por $R_{\mathbb{R}}^{0}:=\sum_{1=1}^{\nu} \mathbb{R} \delta_{i}$ e por $\eta \cdot \theta$ o produto escalar usual entre $\eta, \theta \in R_{\mathbb{R}}^{0}$.

Teorema 3.1.2. ([4], 2.2) Seja $\Psi \subset R^{0}$ um conjunto de raízes isotrópicas positivas. Então existem vetores ortogonais não-nulos $\eta_{1}, \cdots, \eta_{t} \in R_{\mathbb{R}}^{0}$, com $1 \leq t \leq \nu$, tais que

(a) $\Psi=\left(\cup_{i=2}^{t}\left\{\theta \in R^{0} \mid \theta \cdot \eta_{j}=0\right.\right.$ para $\left.\left.1 \leq j \leq i-1, \quad \theta \cdot \eta_{i}>0\right\}\right) \cup\left\{\theta \in R^{0} \mid \theta \cdot \eta_{1}>\right.$ $0\} \cup\{0\}$,

(b) $0 \neq \eta_{1} \in \sum_{i=1}^{\nu} \mathbb{Z}_{+} \delta_{i} e\left(\cap_{j} H_{\eta_{j}}\right) \cap R^{0}=\{0\}$, onde $H_{\eta_{j}}=\left\{\theta \in R_{\mathbb{R}}^{0} \mid \theta \cdot \eta_{j}=0\right\}$ é o hiperplano ortogonal a $\eta_{j}$, para $j=1, \cdots, t$.

Além disso, dado um conjunto de vetores ortogonais $\left\{\eta_{1}, \cdots, \eta_{t}\right\}$ com $1 \leq t \leq \nu$ satisfazendo (b) e se $\Psi$ é definido como em (a), então $\Psi$ é um conjunto de raízes isotrópicas positivas.

O conjunto positivo standard de raízes isotrópicas é definido em [4] como sendo

$R_{+}^{0}=\left(\cup_{i=2}^{\nu}\left\{\theta \in R \mid\left(\theta, \Lambda_{j}\right)=0\right.\right.$, para $\left.\left.1 \leq j \leq i-1,\left(\theta, \Lambda_{i}\right)>0\right\}\right) \cup\left\{\theta \in R \mid\left(\theta, \Lambda_{1}\right)>0\right\} \cup\{0\}$.

Definição 3.1.3. Um subconjunto $P \subset R$ chama-se sistema de raízes positivo normal se

(i) $P \cap(-P)=\{0\}$,

(ii) $P \cup(-P)=R$,

(iii) para $\alpha, \beta \in P$ e $\alpha+\beta \in R$, então $\alpha+\beta \in P$,

(iv) $P \supset R_{+}^{0}$.

A partir daqui consideramos o sistema de raízes $R=\mathbb{Z}^{2} \cup(\dot{R}+S)$ da seção 2.2 , onde $S$ é um semi-reticulado do $\mathbb{R}^{2}$.

Consideramos os seguintes sistemas de raízes positivos normais de $R$. Provaremos que $P_{1}, P_{2}, P_{3}$ e $P_{4}$ são únicas, a menos de $\mathcal{W}$-equivalência. 


$$
\begin{aligned}
P_{1}= & (\dot{\alpha}+S) \cup R_{+}^{0} \\
P_{2}= & \left\{\dot{\alpha}+n \delta_{1}+m \delta_{2} \mid n m=0(\bmod 2), m=-1 \Rightarrow n \geq 2, m \geq 0 \Rightarrow n \in \mathbb{Z}\right\} \\
& \cup\left\{-\dot{\alpha}+n \delta_{1}+m \delta_{2} \mid n m=0(\bmod 2), m=0 \Rightarrow n \geq 0, m \geq 1 \Rightarrow n \in \mathbb{Z}\right\} \cup R_{+}^{0} \\
P_{3}= & \left\{\dot{\alpha}+n \delta_{1}+m \delta_{2} \mid n m=0(\bmod 2), m \geq 0\right\} \cup\left\{-\dot{\alpha}+n \delta_{1}+m \delta_{2} \mid n m=0(\bmod 2), m>0\right\} \\
& \cup R_{+}^{0} \\
P_{4}= & \left\{\dot{\alpha}+n \delta_{1}+m \delta_{2} \mid n m=0(\bmod 2), m=0 \Rightarrow n \geq 0, m \geq 1 \Rightarrow n \in \mathbb{Z}\right\} \\
& \cup\left\{-\dot{\alpha}+n \delta_{1}+m \delta_{2} \mid n m=0(\bmod 2), m=0 \Rightarrow n>0, m \geq 1 \Rightarrow n \in \mathbb{Z}\right\} \cup R_{+}^{0}
\end{aligned}
$$

Ilustramos abaixo os $\dot{\alpha}$-planos de $P_{1}, P_{2}, P_{3}$ e $P_{4}$.

$$
P_{1}
$$

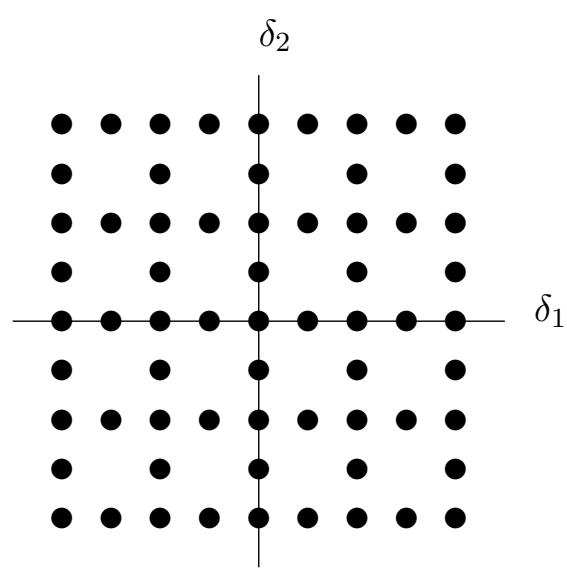

$P_{3}$

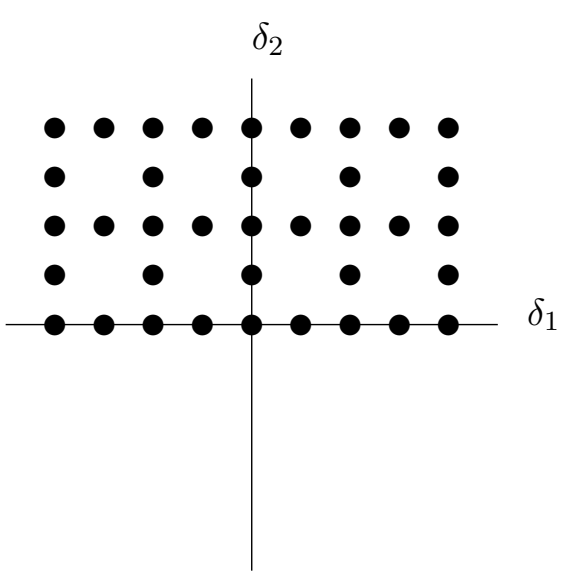

$P_{2}$

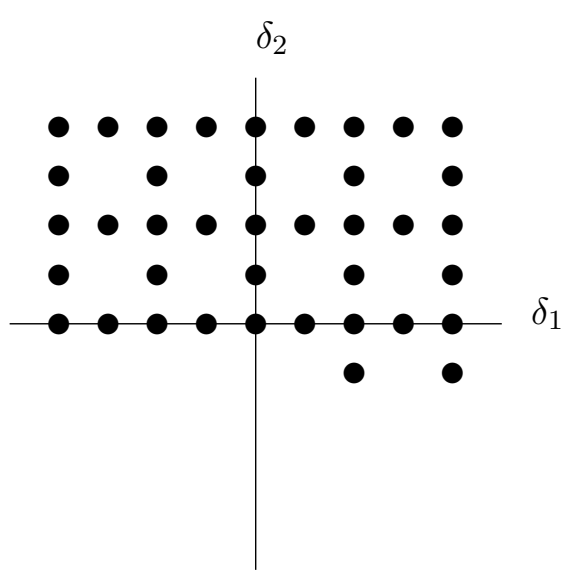

$P_{4}$

$\delta_{2}$

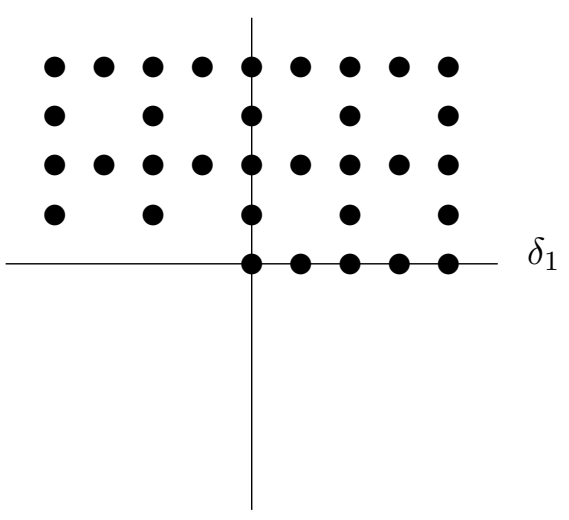


Teorema 3.1.4. (i) Os sistemas de raízes positivos normais $P_{1}, P_{2}, P_{3}$ e $P_{4}$ são $\mathcal{W}$-nãoequivalentes.

(ii) Se $P$ é um sistema de raízes positivo normal em $R$, então existe $w \in \mathcal{W}$ tal que $w P=P_{i}$ para algum $i \in\{1,2,3,4\}$.

Prova: (i) Suponhamos que $P_{2}$ seja $\mathcal{W}$-equivalente a $P_{i}$, para algum $i \in\{1,3,4\}$. Então, existe um $w \in \mathcal{W}$ tal que $w\left(P_{2}\right)=P_{i}$. Pela Proposição 2.1.10 $w$ pode ser escrito como $w=\dot{w} h$ para algum $\dot{w} \in \dot{\mathcal{W}}$ e $h \in H$. Sejam $\dot{\alpha}+2 \delta_{1}-\delta_{2}$ e $-\dot{\alpha}+2 \delta_{1}+\delta_{2}$ dois elementos em $P_{2}$. Então

$$
\begin{aligned}
h\left(\dot{\alpha}+2 \delta_{1}-\delta_{2}\right) & =\dot{\alpha}+2 \delta_{1}-\delta_{2}+2 k_{1} \delta_{1}+2 k_{2} \delta_{2} \\
& =\dot{\alpha}+2\left(k_{1}+1\right) \delta_{1}+\left(2 k_{2}-1\right) \delta_{2}, \\
h\left(-\dot{\alpha}+2 \delta_{1}+\delta_{2}\right) & =-\dot{\alpha}+2 \delta_{1}+\delta_{2}-2 k_{1} \delta_{1}-2 k_{2} \delta_{2} \\
& =-\dot{\alpha}-2\left(k_{1}-1\right)-\left(2 k_{2}-1\right) \delta_{2} .
\end{aligned}
$$

Os elementos $\dot{w} h\left(\dot{\alpha}-\delta_{1}-2 \delta_{2}\right)$ e $\dot{w} h\left(\dot{\alpha}-\delta_{1}+2 \delta_{2}\right)$ possuem sinais opostos em $\delta_{2}$ e portanto não pertencem ambos a $P_{1}, P_{3}$ ou $P_{4}$, contradição. Portanto, $P_{2}$ não é $\mathcal{W}$-equivalente a $P_{i}$ para algum $i \in\{1,3,4\}$.

Agora provaremos que $P_{3}$ não pode ser $\mathcal{W}$-equivalente a $P_{1}$ ou $P_{4}$. Assumimos que existe um $w \in \mathcal{W}$ tal que $w\left(P_{3}\right)=P_{i}$, para $i=1$ ou 4 . Seja $w=\dot{w} h$ para algum $\dot{w} \in \dot{\mathcal{W}}$ e $h \in H$. Consideramos os elementos $\dot{\alpha}-\delta_{1}$ e $-\dot{\alpha}+\delta_{1}$. Aplicando $h$ a esses elementos temos

$$
\begin{aligned}
h\left(\dot{\alpha}-\delta_{1}\right) & =\dot{\alpha}-\delta_{1}+2 k_{1} \delta_{1}+2 k_{2} \delta_{2} \\
& =\dot{\alpha}+\left(2 k_{1}-1\right) \delta_{1}+2 k_{2} \delta_{2}, \\
h\left(-\dot{\alpha}+\delta_{1}\right) & =-\dot{\alpha}+\delta_{1}-2 k_{1} \delta_{1}-2 k_{2} \delta_{2} \\
& =-\dot{\alpha}-\left(2 k_{1}-1\right) \delta_{1}-2 k_{2} \delta_{2} .
\end{aligned}
$$

Desde que $\dot{w} h\left(\dot{\alpha}-\delta_{1}\right)$ e $\dot{w} h\left(-\dot{\alpha}+\delta_{1}\right)$ possuem sinais opostos em $\delta_{1}$ e $\delta_{2}$, ambos não pertencem a $P_{1}$ ou $P_{4}$. Portanto, $P_{3}$ não é $\mathcal{W}$-equivalente a $P_{1}$ ou $P_{4}$.

Finalmente, provaremos que $P_{1}$ não é $\mathcal{W}$-equivalente a $P_{4}$. Assumimos novamente que existe $w \in \mathcal{W}$ tal que $w\left(P_{4}\right)=P_{1}$. Então existem $\dot{w} \in \dot{\mathcal{W}}$ e $h \in H$ tais que $w=\dot{w} h$ e 
consideramos os elementos $\dot{\alpha}+\delta_{1}$ e $-\dot{\alpha}+\delta_{1}$ em $P_{4}$.

$$
\begin{aligned}
h\left(\dot{\alpha}+\delta_{1}\right) & =\dot{\alpha}+\delta_{1}+2 k_{1} \delta_{1}+2 k_{2} \delta_{2} \\
& =\dot{\alpha}+\left(2 k_{1}+1\right) \delta_{1}+2 k_{2} \delta_{2}, \\
h\left(-\dot{\alpha}+\delta_{1}\right) & =-\dot{\alpha}+\delta_{1}-2 k_{1} \delta_{1}-2 k_{2} \delta_{2} \\
= & -\dot{\alpha}-\left(2 k_{1}-1\right) \delta_{1}-2 k_{2} \delta_{2} .
\end{aligned}
$$

Então ou $\dot{w} h\left(\dot{\alpha}+\delta_{1}\right)$ ou $\dot{w} h\left(-\dot{\alpha}+\delta_{1}\right)$ não pertence a $P_{1}$. Assim, provamos o item (i).

(ii) Seja $P$ um sistema de raízes positivo normal em $R$. Seja $P^{\prime}=P \backslash R_{+}^{0}$. Denotamos por $R_{i}=\dot{R}+\mathbb{Z} \delta_{i}$, para $i=1,2$. Sejam $W_{1}$ e $W_{2}$ os grupos de Weyl de $R_{1}$ e $R_{2}$, respectivamente. Consideramos $P \cap R_{2}$ que será um sistema positivo para $R_{2}$ e, portanto, existe $w \in W_{2}$ tal que $w\left(P^{\prime} \cap R_{2}\right)=R_{2}^{1}$ ou $R_{2}^{2}$, onde $R_{2}^{1}=\left\{\dot{\alpha}+m \delta_{2} \mid m \in \mathbb{Z}\right\}$ e $R_{2}^{2}=\left\{\dot{\alpha}+n \delta_{2},-\dot{\alpha}+m \delta_{2} \mid n \geq 0, m>0\right\}$.

Caso 1: $w\left(P^{\prime} \cap R_{2}\right)=R_{2}^{1}$.

Como $w(P)$ é fechado para soma e $R_{+}^{0} \subset w(P)$ temos $w\left(P^{\prime}\right)=\left(R_{2}^{1}+R_{+}^{0}\right) \cap R$ e $w(P)=P_{1}$. Portanto, $P$ será $W$-equivalente a $P_{1}$.

Caso 2: $w\left(P^{\prime} \cap R_{2}\right)=R_{2}^{2}$.

Neste caso $w\left(P^{\prime}\right) \supset\left(R_{2}^{2}+R_{+}^{0}\right) \cap R$. Por outro lado, o elemento $-\dot{\alpha}+\delta_{2} \in w(P)$ e como $P$ é um sistema de raízes positivo temos que

$$
-w(P) \cap\left(-\dot{\alpha}+\delta_{2}+R_{+}^{0}\right) \cap R=\emptyset
$$

Isto implica $w(P) \cap\left(\dot{\alpha}-\delta_{2}-R_{+}^{0}\right) \cap R=\emptyset$ e portanto $w(P) \cap\left(\dot{\alpha}+R_{+}^{0}\right) \cap R \subset\left(\dot{\alpha}-\delta_{2}+R_{+}^{0}\right) \cap R$. Assim, $\mathrm{P}$ deverá ser equivalente a $P_{2}, P_{3}$ ou $P_{4}$.

Consideramos o subconjunto $w(P) \cap R_{1}$ que é um sistema de raízes positivo de $R_{1}$. Analogamente a $R_{2}$, existe um elemento $w^{\prime} \in W_{1}$ tal que $w^{\prime}\left(w\left(P^{\prime}\right) \cap R_{1}\right)=R_{1}^{1}$ ou $R_{1}^{2}$, onde $R_{1}^{1}=\left\{\dot{\alpha}+m \delta_{1} \mid m \in \mathbb{Z}\right\}$ e $R_{1}^{2}=\left\{\dot{\alpha}+n \delta_{1},-\dot{\alpha}+m \delta_{1} \mid n \geq 0, m>0\right\}$.

Caso 2.1: $w^{\prime}\left(\left(w(P) \backslash R_{+}^{0}\right) \cap R_{1}\right)=R_{1}^{1}$ e $\dot{\alpha}+2 n \delta_{1}-\delta_{2} \in w(P)$, para algum $n>0$.

Daí, $\left(R_{2}^{2}+\mathbb{Z} \delta_{1}\right) \cap R \subset w(P)$. Adicionando elementos adequados de $R_{+}^{0}$ a $\dot{\alpha}+2 n \delta_{1}-\delta_{2}$, todos os pontos da forma $\dot{\alpha}+2 k \delta_{1}-\delta_{2}$, para $k>n$, estariam em $w(P)$ e os pontos da forma $\dot{\alpha}+2 k \delta_{1}-\delta_{2}$, para $k<n$, estariam no eixo $\delta_{1}$. Transladando $w(P) \cap\left(\dot{\alpha}+R_{+}^{0}\right)$ para à esquerda, por uma escolha adequada de um múltiplo de $2 \delta_{1}$, concluímos que $P$ será $W$-equivalente a $P_{2}$. 
Caso 2.2: $w^{\prime}\left(\left(w(P) \backslash R_{+}^{0}\right) \cap R_{1}\right)=R_{1}^{1}$ e $\dot{\alpha}+2 n \delta_{1}-\delta_{2} \notin w(P)$, para todo $n>0$.

Neste caso, como $\left(R_{2}^{2}+\mathbb{Z} \delta_{1}\right) \cap R \subset w(P), P$ será $W$-equivalente a $P_{3}$.

Caso 2.3: $w^{\prime}\left(\left(w(P) \backslash R_{+}^{0}\right) \cap R_{1}\right)=R_{1}^{2}$.

Neste caso $\dot{\alpha}+2 n \delta_{1}-\delta_{2}$ não deve pertencer a $w(P)$, para todo $n>0$, caso contrário adicionando elementos adequados de $R_{+}^{0}$ a $\dot{\alpha}+2 n \delta_{1}-\delta_{2}$ teríamos pontos no eixo $\delta_{1}$ que não pertencem a $w^{\prime}\left(\left(w(P) \backslash R_{+}^{0}\right) \cap R_{1}\right)=R_{1}^{2}$, portanto, $\dot{\alpha}+2 n \delta_{1}-\delta_{2} \notin w(P)$, para todo $n>0$ e, como $\left(R_{2}^{2}+\mathbb{N} \delta_{1}\right) \cap R \subset w(P), P$ será equivalente a $P_{4}$.

\subsection{Subálgebra de Kac-Moody afim}

Consideramos o seguinte subconjunto $\Sigma_{1}=\left\{n \delta_{1} \mid n \in \mathbb{Z}\right\}$ de $R^{0}$. Seja

$$
A:=\left(s l_{2}(\mathbb{C}) \otimes\left(\sum_{\sigma \in \Sigma_{1}} J^{\sigma}\right)\right) \oplus \mathbb{C}_{\mathbf{1}} \oplus \mathbb{C d}_{\mathbf{1}}
$$

Para $X \otimes x^{\xi}, Y \otimes x^{\tau} \in s l_{2}(\mathbb{C}) \otimes\left(\sum_{\sigma \in \Sigma_{1}} J^{\sigma}\right)$ temos

$$
\left[X \otimes x^{\xi}, Y \otimes x^{\tau}\right]=[X, Y] \otimes x^{\xi+\tau}+(A, B)\left[L_{x^{\xi}}, L_{x^{\tau}}\right]+\sum_{i=1,2}\left(d_{i}\left(X \otimes x^{\xi}\right), Y \otimes x^{\tau}\right) c_{i}
$$

Agora $\mathbf{d}_{\mathbf{2}}\left(A \otimes x^{\xi}\right)=0$, pois $\xi \in \Sigma_{1}$ e $\left[L_{x \xi}, L_{x^{\tau}}\right]=0$, pelo Lema 2.2.1, como $\xi+\tau \in S$. Logo

$$
\left[X \otimes x^{\xi}, Y \otimes x^{\tau}\right]=[X, Y] \otimes x^{\xi+\tau}+\left(\mathbf{d}_{\mathbf{1}}\left(X \otimes x^{\xi}\right), Y \otimes x^{\tau}\right) \mathbf{c}_{\mathbf{1}}
$$

e como $\mathbf{c}_{\mathbf{1}}$ é um elemento central e $\mathbf{d}_{\mathbf{1}}$ age como derivação do grau, $A$ é uma subálgebra de $\mathcal{L}(J)$.

O subespaço $\left(\sum_{\sigma \in \Sigma_{1}} J^{\sigma}\right)$ de $J$ é uma álgebra comutativa, associativa com unidade, gerada por $x^{(1,0)}$ e $x^{(-1,0)}$ e isomorfa à álgebra de polinômios de Laurent gerada por uma variável e sua inversa. Portanto, $A$ é isomorfa à álgebra de Kac-Moody afim $\left.s l_{2} \hat{(} \mathbb{C}\right)$.

Denotamos $\Sigma_{1}^{ \pm}=\left\{n \delta_{1} \mid n \in \mathbb{Z}, \pm n>0\right\}$. Assim podemos decompor $A$ em $A=$ 
$A_{+} \oplus A_{0} \oplus A_{-}$, onde

$$
\begin{aligned}
& A_{+}=\left(\mathbb{C} e_{+} \otimes x^{0}\right) \oplus s l_{2}(\mathbb{C}) \otimes\left(\sum_{\sigma \in \Sigma_{1}^{+}} J^{\sigma}\right), \\
& A_{0}=\left(\mathbb{C} h \otimes x^{0}\right) \oplus \mathbb{C c}_{\mathbf{1}} \oplus \mathbb{C d}_{\mathbf{1}}, \\
& A_{-}=\left(\mathbb{C} e_{-} \otimes x^{0}\right) \oplus s l_{2}(\mathbb{C}) \otimes\left(\sum_{\sigma \in \Sigma_{1}^{-}} J^{\sigma}\right) .
\end{aligned}
$$

Denotamos $\Sigma_{2}^{ \pm}=\left\{n \delta_{1}+m \delta_{2} \mid n, m \in \mathbb{Z}, \pm m>0\right\}$ e definimos o seguinte subespaço de $\mathcal{L}(J)$ :

$$
\mathcal{L}_{ \pm 2}(J)=s l_{2}(\mathbb{C}) \otimes\left(\sum_{\sigma \in \Sigma_{2}^{ \pm} \cap S} J^{\sigma}\right) \oplus\left(\sum_{\sigma \in \Sigma_{2}^{ \pm} \cap S^{\perp}} \mathbb{C}\left[L_{x^{\delta_{1}}}, L_{x^{\sigma-\delta_{1}}}\right]\right) .
$$

Seja $\mathcal{L}_{ \pm}(J)=A_{ \pm}+\mathcal{L}_{ \pm 2}(J)$. Então,

$$
\mathcal{L}(J)=\mathcal{L}_{+}(J) \oplus \mathcal{L}_{0}(J) \oplus \mathcal{L}_{-}(J),
$$

lembrando que $\mathcal{L}_{0}(J)=\left(\mathcal{G}_{0}(J) \cap \mathcal{G}^{0}(J)\right) \oplus \mathcal{C} \oplus \mathcal{D}$.

Seja $\lambda \in \mathcal{L}_{0}(J)^{*}$ e denotamos por $\hat{\lambda}$ a restrição de $\lambda$ a $A_{0}$.

Consideramos o módulo de Verma associado a $\hat{\lambda}$ para $A$,

$$
\hat{M}(\hat{\lambda})=U(A) \otimes_{U\left(A_{+} \oplus A_{0}\right)} \mathbb{C} v_{\lambda}
$$

induzido pelo $A_{+} \oplus A_{0}$-módulo unidimensinal $\mathbb{C} v_{\lambda}$, com a ação definida por $(x+h) v_{\lambda}=$ $\lambda(h) v_{\lambda}$, para todos $x \in A_{+}$e $h \in A_{0}$.

\subsection{Base e Ordem}

A álgebra TKK afim estendida da seção 2.2 possui uma base formada pelos seguintes elementos:

$$
\begin{gathered}
e_{ \pm}(m, n)= \begin{cases}e_{ \pm} \otimes x^{(m, n)} & , \text { se }(m, n) \in S, \\
0 & , \text { se }(m, n) \in S^{\perp},\end{cases} \\
h(m, n)= \begin{cases}h \otimes x^{(m, n)} & , \text { se }(m, n) \in S \\
2\left[L_{x^{\delta_{1}}}, L_{x^{(m, n)-\delta_{1}}}\right] & , \text { se }(m, n) \in S^{\perp} .\end{cases}
\end{gathered}
$$


Então $\left\{e_{ \pm}(m, 0), h(m, 0) \mid m \in \mathbb{Z}\right\}$ forma uma base do subespaço $s l_{2}(\mathbb{C}) \otimes\left(\sum_{\sigma \in \Sigma_{1}} J^{\sigma}\right)$ de A.

Definimos uma ordem em $\left\{e_{ \pm}(m, 0), h(m, 0) \mid m \in \mathbb{Z}\right\}$. Para isso consideremos dois elementos $x_{1}\left(m_{1}, 0\right), x_{2}\left(m_{2}, 0\right) \in\left\{e_{ \pm}(m, 0), h(m, 0) \mid m \in \mathbb{Z}\right\}$. Então,

$$
x_{1}\left(m_{1}, 0\right)<x_{2}\left(m_{2}, 0\right) \text { se ou } m_{1}<m_{2} \text { ou } m_{1}=m_{2} \text { e } \beta_{1}<\beta_{2}
$$

onde $\beta_{1}$ e $\beta_{2}$ são as raízes associadas aos elementos $x_{1}$ e $x_{2}$, respectivamente.

O conjunto $\left\{\mathbf{c}_{\mathbf{1}}, \mathbf{d}_{\mathbf{1}}, e_{+}(m, 0), h(m, 0) \mid m \geq 0\right\} \cup\left\{e_{-}(m, 0) \mid m>0\right\}$ forma uma base de $A_{+} \oplus A_{0}$ e $\left\{e_{-}(0,0)\right\} \cup\left\{e_{ \pm}(m, 0), h(m, 0) \mid m<0\right\}$ forma uma base de $A_{-}$.

Seja $B\left(\mathcal{L}_{-}(J)\right):=\left\{e_{-}(0,0)\right\} \cup\left\{e_{ \pm}(m, 0), h(m, 0) \mid m<0\right\} \cup\left\{e_{ \pm}(m, n) \mid(m, n) \in\right.$ $S, n<0\} \cup\left\{h(m, n) \mid(m, n) \in \mathbb{Z}^{2}, n<0\right\}$ uma base de $\mathcal{L}_{-}(J)$. Para dois elementos $x_{1}\left(m_{1}, n_{1}\right), x_{2}\left(m_{2}, n_{2}\right) \in B\left(\mathcal{L}_{-}(J)\right)$ dizemos que

$$
x_{1}\left(m_{1}, n_{1}\right)<x_{2}\left(m_{2}, n_{2}\right) \text { se ou } n_{1}<n_{2} \text { ou } n_{1}=n_{2} \text { e } x_{1}\left(m_{1}, 0\right)<x_{2}\left(m_{2}, 0\right) .
$$

Isto define uma ordem total em $B\left(\mathcal{L}_{-}(J)\right)$.

Um base de $U\left(\mathcal{L}_{-}(J)\right)$ consiste de monômios da forma

$$
x_{1}\left(m_{1}, n_{1}\right) x_{2}\left(m_{2}, n_{2}\right) \cdots x_{r}\left(m_{r}, n_{r}\right)
$$

onde $x_{i}\left(m_{i}, n_{i}\right) \in B\left(\mathcal{L}_{-}(J)\right)$, para $i=1, \cdots, r$, e $x_{1}\left(n_{1}, m_{1}\right) \leq x_{2}\left(m_{2}, n_{2}\right) \leq \cdots \leq$ $x_{r}\left(m_{r}, n_{r}\right)$.

Denotamos por

$$
x_{i, m, n, p}:=x_{i_{1}}\left(m_{1}, n_{1}\right)^{p_{1}} x_{i_{2}}\left(m_{2}, n_{2}\right)^{p_{2}} \cdots x_{i_{r}}\left(m_{r}, n_{r}\right)^{p_{r}}
$$

onde $i=\left(i_{1}, \cdots, i_{r}\right), m=\left(m_{1}, \cdots, m_{r}\right), n=\left(n_{1}, \cdots, n_{r}\right), p=\left(p_{1}, \cdots, p_{r}\right)$ e $m_{j} \leq-1$ se $n_{j}=0$ e $m_{j} \in \mathbb{Z}$ se $n_{j} \leq-1$ e

$$
x_{i_{j}}= \begin{cases}e_{-} & , \text {se } m_{j}=n_{j}=0 \\ e_{-}, e_{+} \text {ou } h & , \text { se } m_{j} \leq-1 \text { e } n_{j}=0 \\ e_{-}, e_{+} \text {ou } h & , \text { se } n_{j} \leq-1\end{cases}
$$

Quando $r=0$, o monômio $x_{i, m, n, p}$ denotará o elemento identidade de $U\left(\mathcal{L}_{-}(J)\right)$. Desta maneira $B\left(U\left(\mathcal{L}_{-}(J)\right)\right):=\left\{x_{i, m, n, p}\right\} \cup\{1\}$ forma uma base de $U\left(\mathcal{L}_{-}(J)\right)$. 
Sejam $B_{1}=\left\{x_{i, m, n, p} \in B\left(U\left(\mathcal{L}_{-}(J)\right)\right) \mid n_{j}=0, \forall j\right\} \cup\{1\}$ e $B_{2}=\left\{x_{i, m, n, p} \in B\left(U\left(\mathcal{L}_{-}(J)\right)\right) \mid\right.$ $\left.n_{j} \neq 0, \forall j\right\} \cup\{1\}$. Então $B\left(U\left(\mathcal{L}_{-}(J)\right)\right)=B_{2} B_{1}=\left\{b_{2} b_{1} \mid b_{i} \in B_{i}, i=1,2\right\}$

Definimos uma ordem total em $B_{1}$ e $B_{2}$ como em [3]. Dizemos que

$$
x_{i, m, n, p}=x_{i_{1}}\left(m_{1}, n_{1}\right)^{p_{1}} \cdots x_{i_{r}}\left(m_{r}, n_{r}\right)^{p_{r}}<x_{j_{1}}\left(k_{1}, l_{1}\right)^{q_{1}} \cdots x_{j_{s}}\left(k_{s}, l_{s}\right)^{q_{s}}=x_{j, k, l, q}
$$

se, e somente se, existe um $\gamma \geq 0$ tal que

$$
x_{i_{\gamma}}\left(m_{\gamma}, n_{\gamma}\right)^{p_{\gamma}} \cdots x_{i_{r}}\left(m_{r}, n_{r}\right)^{p_{r}}=x_{j_{\gamma}}\left(k_{\gamma}, l_{\gamma}\right)^{q_{\gamma}} \cdots x_{j_{s}}\left(k_{s}, l_{s}\right)^{q_{s}}
$$

e ou $x_{i_{\gamma-1}}\left(m_{\gamma-1}, n_{\gamma-1}\right)^{p_{\gamma-1}}<x_{j_{\gamma-1}}\left(k_{\gamma-1}, l_{\gamma-1}\right)^{q_{\gamma-1}}$ ou $x_{i_{\gamma-1}}\left(m_{\gamma-1}, n_{\gamma-1}\right)^{p_{\gamma-1}}=x_{j_{\gamma-1}}\left(k_{\gamma-1}, l_{\gamma-1}\right)^{q_{\gamma-1}}$ e $p_{\gamma-1}>q_{\gamma-1}$.

Caso $r>s$ e $x_{i_{r-s+1}}\left(m_{r-s+1}, n_{r-s+1}\right)^{p_{r-s+1}} \cdots x_{i_{r}}\left(m_{r}, n_{r}\right)^{p_{r}}=x_{j_{1}}\left(k_{1}, l_{1}\right)^{q_{1}} \cdots x_{j_{s}}\left(k_{s}, l_{s}\right)^{q_{s}}$ convencionamos que $x_{i, m, n, p}<x_{j, k, l, q}$. Isso significa que $x_{i_{r-s}}\left(m_{r-s}, n_{r-s}\right)<1$.

Para $b_{2} b_{1}, b_{2}^{\prime} b_{1}^{\prime} \in B\left(U\left(\mathcal{L}_{-}(J)\right)\right)=B_{2} B_{1}=\left\{b_{2} b_{1} \mid b_{i} \in B_{i}, i=1,2\right\}$ dizemos que

$$
b_{2} b_{1}<b_{2}^{\prime} b_{1}^{\prime}
$$

se ou $b_{2}<b_{2}^{\prime}$ ou $b_{2}=b_{2}^{\prime}$ e $b_{1}<b_{1}^{\prime}$. Isso define uma ordem total em $B\left(U\left(\mathcal{L}_{-}(J)\right)\right)$ que estende a ordem definida sobre $B_{1}$ e $B_{2}$.

Para $x_{i, m, n, p} \in U\left(\mathcal{L}_{-}(J)\right)$ denotamos por

$$
x_{i, m, n, p}^{\hat{p_{j}}}=x_{i_{1}}\left(m_{1}, n_{1}\right)^{p_{1}} \cdots x_{i_{j}}\left(m_{j}, n_{j}\right)^{p_{j}-1} \cdots x_{i_{r}}\left(m_{r}, n_{r}\right)^{p_{r}} .
$$

\subsection{Módulo de Verma Clássico}

Lembramos aqui que existem quatro órbitas de subálgebras de Borel que levam a quatro tipos de módulos de Verma. Os casos mais importantes (caso "extremos") são os chamados clássicos e imaginários. Estudaremos aqui o caso clássico.

Seja $P$ um sistema de raízes positivo normal em $R$. Denotamos por

$$
B_{P}=\left(\sum_{\sigma \in P \backslash\{0\}} \mathcal{L}^{\sigma}(J)\right) \oplus \mathcal{H}
$$

a subálgebra de Borel de $\mathcal{L}(J)$ associada a $P$. 
Para $\lambda \in \mathcal{L}_{0}(J)^{*}$, consideramos o módulo tipo Verma associado a $B_{P}$ e $\lambda$,

$$
M_{B_{P}}(\lambda)=U(\mathcal{L}(J)) \otimes_{U\left(B_{P}\right)} \mathbb{C} v_{\lambda}
$$

onde $\mathbb{C} v_{\lambda}$ é um $B_{P}$-módulo unidimensional com a ação $(h+x) v_{\lambda}=\lambda(h) v_{\lambda}$, para todo $h \in \mathcal{H}$ e $x \in \sum_{\sigma \in P \backslash\{0\}} \mathcal{L}^{\sigma}(J)$.

Denotamos por

$$
M(\lambda):=M_{B_{P_{4}}}(\lambda)
$$

o módulo tipo Verma associado a $B_{P_{4}}$ e $\lambda$, chamado módulo de Verma clássico.

Relembramos aqui algumas notações que serão usadas na demonstrações dos resultados dessa seção. Denotamos por $\Omega$ a aplicação de $S$ em $\mathbb{C}$ definida por

$$
\Omega(\sigma)=\frac{1}{2}\left((-1)^{\sigma \cdot \delta_{1}}-(-1)^{\sigma \cdot \delta_{2}}\right) .
$$

Consideramos os elementos da base standard de $\mathcal{L}(J)$ e suas relações:

$$
\begin{gathered}
e_{ \pm}(m, n)= \begin{cases}e_{ \pm} \otimes x^{(m, n)} & , \text { se }(m, n) \in S, \\
0 & , \text { se }(m, n) \in S^{\perp},\end{cases} \\
h(m, n)= \begin{cases}h \otimes x^{(m, n)} & , \text { se }(m, n) \in S \\
2\left[L_{x^{\delta_{1}}}, L_{x^{(m, n)-\delta_{1}}}\right] & , \text { se }(m, n) \in S^{\perp}\end{cases}
\end{gathered}
$$

Para $\sigma, \tau \in \mathbb{Z}^{2}$, temos

$$
[h(\sigma), h(\tau)]= \begin{cases}2 \Omega(\tau) h(\sigma+\tau) & , \text { se } \sigma \in S^{\perp}, \tau \in S, \\ -4 \delta_{\sigma+\tau, 0}\left(\sigma \cdot \delta_{1} \mathbf{c}_{1}+\sigma \cdot \delta_{2} \mathbf{c}_{\mathbf{2}}\right) & , \text { se } \sigma, \tau \in S^{\perp}, \\ 4 \delta_{\sigma+\tau, 0}\left(\sigma \cdot \delta_{1} \mathbf{c}_{\mathbf{1}}+\sigma \cdot \delta_{2} \mathbf{c}_{\mathbf{2}}\right) & , \text { se } \sigma, \tau \in S, \sigma+\tau \in S, \\ 2 \Omega(\tau) h(\sigma+\tau) & , \text { se } \sigma, \tau \in S, \sigma+\tau \in S^{\perp} .\end{cases}
$$

Para $\sigma \in \mathbb{Z}^{2}$ e $\tau \in S$ temos

$$
\left[h(\sigma), e_{ \pm}(\tau)\right]=\left\{\begin{array}{l} 
\pm 2 e_{ \pm}(\sigma+\tau), \text { se } \sigma \in S \\
2 \Omega(\tau) e_{ \pm}(\sigma+\tau), \text { se } \sigma \in S^{\perp}
\end{array}\right.
$$

Para $\sigma, \tau \in S$ temos

$$
\begin{aligned}
& {\left[e_{ \pm}(\sigma), e_{ \pm}(\tau)\right]=0} \\
& {\left[e_{+}(\sigma), e_{-}(\tau)\right]= \begin{cases}\Omega(\tau) h(\sigma+\tau) & , \text { se } \sigma+\tau \in S^{\perp} \\
h(\sigma+\tau)+2 \delta_{\sigma+\tau, 0}\left(\sigma \cdot \delta_{1} \mathbf{c}_{1}+\tau \cdot \delta_{2} \mathbf{c}_{2}\right) & , \text { se } \sigma+\tau \in S\end{cases} }
\end{aligned}
$$


Seja $z \in s l_{2}(\mathbb{C}) \otimes\left(\sum_{\sigma \in \Sigma_{1}} J^{\sigma}\right)$, então podemos escrevê-lo da seguinte forma:

$$
z=\sum u_{i} x_{i}\left(m_{i}, 0\right)
$$

onde $x_{i}\left(m_{i}, 0\right) \in\left\{e_{ \pm}(m, 0), h(m, 0) \mid m \in \mathbb{Z}\right\}$ e $u_{i} \in \mathbb{C}$ e denotamos por

$$
z(n):=\sum u_{i} x_{i}\left(m_{i}, n\right) .
$$

Denotamos por $\mathcal{L}^{(k)}:=\left\{x \in \mathcal{L}(J) \mid\left[\mathbf{d}_{\mathbf{2}}, x\right]=k x\right\}$.

Lema 3.4.1. Seja $x_{i, m, n, p}=x_{i_{1}}\left(m_{1}, n_{1}\right)^{p_{1}} \cdots x_{i_{k}}\left(m_{k}, n_{k}\right)^{p_{k}}$, com $n_{j} \leq-1$, para $1 \leq j \leq k$. Se $z \in s l_{2}(\mathbb{C}) \otimes\left(\sum_{\sigma \in \Sigma_{1}} J^{\sigma}\right)$ e $n \in \mathbb{Z}$ é tal que $n_{j}<n$, para todo $j$, então

$$
\left[z(-n), x_{i, m, n, p}\right] \in U\left(\mathcal{L}_{-2}(J)\right) \mathcal{L}_{-2}(J)
$$

Prova: Seguindo [3], o operador $a d(z(-n))$ age como uma derivação sobre $U\left(\mathcal{L}_{-2}(J)\right)$, $\log \mathrm{O}$

$$
\left[z(-n), x_{i, m, n, p}\right]=\sum_{j=1}^{k} x_{i_{1}}\left(m_{1}, n_{2}\right)^{p_{1}} \cdots\left[z(-n), x_{i_{j}}\left(m_{j}, n_{j}\right)^{p_{j}}\right] \cdots x_{i_{k}}\left(m_{k}, n_{k}\right)^{p_{k}}
$$

Agora

$$
\left[z(-n), x_{i_{j}}\left(m_{j}, n_{j}\right)^{p_{j}}\right]=\sum_{a=0}^{p_{j}-1} x_{i_{j}}\left(m_{j}, n_{j}\right)^{a}\left[z(-n), x_{i_{j}}\left(m_{j}, n_{j}\right)\right] x_{i_{j}}\left(m_{j}, n_{j}\right)^{p_{j}-1-a}
$$

e

$$
\left[z(-n), x_{i_{j}}\left(m_{j}, n_{j}\right)\right]=\sum_{l} u_{l}\left[x_{l}\left(m_{l},-n\right), x_{i_{j}}\left(m_{j}, n_{j}\right)\right]
$$

Devido às relações $(3.2),(3.3)$ e $(3.4)$ dos elementos da base de $\mathcal{L}(J)$ temos

$$
\left[x_{l}\left(m_{l},-n\right), x_{i_{j}}\left(m_{j}, n_{j}\right)\right]=u_{l}^{\prime} y\left(m_{1}+m_{j},-n+n_{j}\right)
$$

para $u_{l}^{\prime} \in \mathbb{C}$ e para algum $y \in\left\{e_{ \pm}, h\right\}$. Como $n_{j}-n<0$, para todo $j$, concluímos que $\left[z(-n), x_{i, m, n, p}\right] \in U\left(\mathcal{L}_{-2}(J)\right) \mathcal{L}_{-2}(J)$

Lema 3.4.2. Sejam $n$ um inteiro negativo e $z \in A_{\beta+q \delta_{1}}$, com $q \neq 0$ e $\beta \in\{\dot{\alpha}, 0\}$. Então

$$
\left[z(-n), x_{i_{1}}\left(m_{1}, n\right)^{p_{1}} \cdots x_{i_{r}}\left(m_{r}, n\right)^{p_{r}}\right]=y+\sum_{j=1}^{r} u_{j} x_{i, m, n, p}^{\hat{j}}\left[z(-n), x_{i_{j}}\left(m_{j}, n\right)\right]
$$

onde $y \in U\left(\mathcal{L}_{-2}(J)\right) \mathcal{L}_{-2}(J)$ e $x_{i, m, n, p}=x_{i_{1}}\left(m_{1}, n\right)^{p_{1}} \cdots x_{i_{r}}\left(m_{r}, n\right)^{p_{r}}$. 
Prova: Analogamente a [3],

$$
\begin{aligned}
{[z(-n),} & \left.x_{i_{1}}\left(m_{1}, n\right)^{p_{1}} \cdots x_{i_{r}}\left(m_{r}, n\right)^{p_{r}}\right]=\sum_{j=1}^{r} x_{i_{j}}\left(m_{1}, n\right)^{p_{1}} \cdots\left[z(-n), x_{i_{j}}\left(m_{j}, n\right)^{p_{j}}\right] \cdots x_{i_{r}}\left(m_{r}, n\right)^{p_{r}} \\
= & \sum_{j=1}^{r} \sum_{a=0}^{p_{j}-1} x_{i_{j}}\left(m_{1}, n\right)^{p_{1}} \cdots x_{i_{j}}\left(m_{j}, n\right)^{a}\left[z(-n), x_{i_{j}}\left(m_{j}, n\right)\right] x_{i_{j}}\left(m_{j}, n\right)^{p_{j}-1-a} \cdots x_{i_{r}}\left(m_{r}, n\right)^{p_{r}} \\
= & \sum_{j=1}^{r} \sum_{a=0}^{p_{j}-1} x_{i_{j}}\left(m_{1}, n\right)^{p_{1}} \cdots x_{i_{j}}\left(m_{j}, n\right)^{a}\left[\left[z(-n), x_{i_{j}}\left(m_{j}, n\right)\right], x_{i_{j}}\left(m_{j}, n\right)^{p_{j}-1-a} \cdots x_{i_{r}}\left(m_{r}, n\right)^{p_{r}}\right] \\
& +\sum_{j=1}^{r} \sum_{a=0}^{p_{j}-1} x_{i_{j}}\left(m_{1}, n\right)^{p_{1}} \cdots x_{i_{j}}\left(m_{j}, n\right)^{a} x_{i_{j}}\left(m_{j}, n\right)^{p_{j}-1-a} \cdots x_{i_{r}}\left(m_{r}, n\right)^{p_{r}}\left[z(-n), x_{i_{j}}\left(m_{j}, n\right)\right] \\
= & \sum_{j=1}^{r} \sum_{a=0}^{p_{j}-1} x_{i_{j}}\left(m_{1}, n\right)^{p_{1}} \cdots x_{i_{j}}\left(m_{j}, n\right)^{a}\left[\left[z(-n), x_{i_{j}}\left(m_{j}, n\right)\right], x_{i_{j}}\left(m_{j}, n\right)^{p_{j}-1-a} \cdots x_{i_{r}}\left(m_{r}, n\right)^{p_{r}}\right] \\
& +\sum_{j=1}^{r} \sum_{a=0}^{p_{j}-1} x_{i_{j}}\left(m_{1}, n\right)^{p_{1}} \cdots x_{i_{j}}\left(m_{j}, n\right)^{p_{j}-1} \cdots x_{i_{r}}\left(m_{r}, n\right)^{p_{r}}\left[z(-n), x_{i_{j}}\left(m_{j}, n\right)\right] .
\end{aligned}
$$

Denotamos por $y$ o primeiro termo do lado direito da última equação acima. Já que $\left[z(-n), x_{i_{j}}\left(m_{j}, n\right)\right] \in \mathcal{L}^{(0)}$ e $n<0$, pelo Lema 3.4 .1 temos que $y \in U\left(\mathcal{L}_{-2}(J)\right) \mathcal{L}_{-2}(J)$ e assim provamos o lema.

Lema 3.4.3. Seja $I=\left\{i_{1}, \cdots, i_{n}\right\}$ um subconjunto finito de $\mathbb{Z}$. Então existe um elemento $z(2 K, 0) \in\left(s_{2}(\mathbb{C}) \otimes J\right) \cap A_{\beta}$, para alguma raiz $\beta$ de $A$, satisfazendo

(i) $\left[z(2 K, 0), x_{i_{1}}\left(m_{1}, 0\right)\right] \neq 0$,

(ii) se $\beta+\beta_{i_{j}}$ é uma raiz de $A$, então $\beta+\beta_{i_{j}}<\beta_{i_{k}}$, para todo $1 \leq k \leq n$,

(iii) $\left[z(2 K, r), x_{i_{j}}\left(m_{j},-r\right)\right]=\left[z(2 K, 0), x_{i_{j}}\left(m_{j}, 0\right)\right]$ ou $-2 x_{i_{j}}\left(2 K+m_{j}, 0\right)$, para todo $r \in \mathbb{Z}$ $e 1 \leq j \leq n$.

Prova: Para provar o item (i) e (ii) observamos que o elemento $z(m, 0) \in\left(s l_{2}(\mathbb{C}) \otimes J\right) \cap A_{\beta}$ é da forma $z(m, 0)=z \otimes x^{(m, 0)}$. Se $x_{i_{1}}\left(m_{1}, 0\right) \neq 0$, então $0 \neq x_{i_{1}} \in s l_{2}(\mathbb{C})$. Como $s l_{2}(\mathbb{C})$ é simples, existe um elemento $z \in s l_{2}(\mathbb{C})$ tal que $\left[z, x_{i_{1}}\right] \neq 0$. Tomando $m$ suficientemente 
grande garantimos que $\left[z(m, 0), x_{i_{1}}\left(m_{1}, 0\right)\right]=\left[z, x_{i_{1}}\right] \otimes x^{\left(m+m_{1}, 0\right)} \neq 0$ e que $\beta+\beta_{i_{j}}<\beta_{i_{k}}$, para todo $1 \leq k \leq n$.

Para provar o item (iii) necessitamos que $m=2 K$, pois caso contrário se $z \in\left\{e_{ \pm}\right\}$e $(m, n) \in S^{\perp}$ então $z(m, n)=0$. Supondo que $z(2 K, n)=h(2 K, n)$ e $K$ seja suficientemente grande, então

$$
\left[z(2 K, n), e_{ \pm}\left(m_{j},-n\right)\right]= \pm 2 e_{ \pm}\left(2 K+m_{j}, 0\right)
$$

pois $(2 K, n) \in S$ e

$$
\left[z(2 K, n), h\left(m_{j},-n\right)\right]= \begin{cases}0 & , \text { se }\left(m_{j},-n\right) \in S \\ -2 h\left(2 K+m_{j}, 0\right) & , \text { se }\left(m_{j},-n\right) \in S^{\perp}\end{cases}
$$

Supondo que $z(2 K, n)=e_{+}(2 K, n)$ então

$$
\left[z(2 K, n), e_{-}\left(m_{j},-n\right)\right]=h\left(2 K+m_{j}, 0\right)
$$

pois $\left(2 K+m_{j}, 0\right) \in S$ e

$$
\left[z(2 K, n), h\left(m_{j},-n\right)\right]=-2 e_{+}\left(2 K+m_{j}, 0\right) .
$$

independente se $\left(m_{j},-n\right) \in S$ or $\left(m_{j},-n\right) \in S^{\perp}$.

Supondo que $z(2 K, n)=e_{-}(2 K, n)$

$$
\left[z(2 K, n), e_{+}\left(m_{j},-n\right)\right]=-h\left(2 K+m_{j}, 0\right)
$$

pois $\left(2 K+m_{j}, 0\right) \in S$ e

$$
\left[z(2 K, n), h\left(m_{j},-n\right)\right]=2 e_{+}\left(2 K+m_{j}, 0\right) .
$$

independente se $\left(m_{j},-n\right) \in S$ ou $\left(m_{j},-n\right) \in S^{\perp}$.

Portanto, $\left[z(2 K, r), x_{i_{j}}\left(m_{j},-r\right)\right]=\left[z(2 K, 0), x_{i_{j}}\left(m_{j}, 0\right)\right]$ ou $-2 x_{i_{j}}\left(2 K+m_{j}, 0\right)$ para todo $r \in \mathbb{Z}$ e $1 \leq j \leq n$.

Para $N \in M(\lambda)$ denotamos por $N^{(n)}:=\left\{x \in M(\lambda) \mid \mathbf{d}_{\mathbf{2}} x=n x\right\}$ e por $\hat{M}:=\hat{M}(\hat{\lambda})$. Seguindo a linha de [3] para provar o teorema sobre a irredutibilidade do módulo de Verma clássico enunciamos os seguintes lemas:

Lema 3.4.4. Se $M(\lambda)$ é um $\mathcal{L}(J)$-módulo irredutivel, então $\hat{M}$ é um A-módulo irredutível. 
Prova: Supomos que $\hat{M}$ não seja irredutível e denotamos por $\hat{N} \subset \hat{M}$ um $A$-submódulo próprio. Então $1 \otimes v_{\hat{\lambda}} \notin \hat{N}$ e, portanto, $\hat{N}_{\hat{\lambda}}=\{0\}$.

Seja $u \in U\left(\mathcal{L}_{2}(J)\right) \cap U(\mathcal{L}(J))^{(n)}$, para algum $m \in \mathbb{Z}$ e $n>0$. Então $u \hat{N} \subset$ $M(\lambda)^{\left(n+\lambda\left(\mathbf{d}_{2}\right)\right)}=\{0\}$. De fato, seja $v \in \hat{N}$ e $v=\sum_{i} y_{i} \otimes v_{\lambda}$, onde $y_{i} \in U\left(A_{-}\right)$. Então

$$
\begin{aligned}
\mathbf{d}_{\mathbf{2}}(u v) & =\mathbf{d}_{\mathbf{2}}\left(\sum_{i} u y_{i} \otimes v_{\lambda}\right) \\
& =\sum_{i}\left[\mathbf{d}_{\mathbf{2}}, u\right] y_{i} \otimes v_{\lambda}+\sum_{i} u \mathbf{d}_{\mathbf{2}} y_{i} \otimes v_{\lambda} .
\end{aligned}
$$

Mas $\mathbf{d}_{\mathbf{2}}$ comuta como os elementos de $U(A), \log 0$

$$
\begin{aligned}
\mathbf{d}_{\mathbf{2}}(u v) & =\sum_{i} n u y_{i} \otimes v_{\lambda}+\sum_{i} \lambda\left(\mathbf{d}_{\mathbf{2}}\right) u y_{i} \otimes v_{\lambda} \\
& =\left(n+\lambda\left(\mathbf{d}_{\mathbf{2}}\right)\right) u v .
\end{aligned}
$$

Como $n>0$ temos que $M(\lambda)^{\left(n+\lambda\left(\mathbf{d}_{\mathbf{2}}\right)\right)}=\{0\}$.

Assim $U\left(\mathcal{L}_{2}(J)\right) \hat{N}=\oplus_{n \geq 0} U\left(\mathcal{L}_{2}(J)\right)^{(n)} \hat{N}=\hat{N}$ e, pelo Teorema de PBW, temos

$$
\begin{aligned}
U(\mathcal{L}(J)) \hat{N} & =U\left(\mathcal{L}_{-2}\right) U\left(A_{-}\right) U\left(\mathcal{L}_{0}\right) U\left(A_{1}\right) U\left(\left(L_{2}\right)\right) \hat{N} \\
& =U\left(\mathcal{L}_{-2}\right) U\left(A_{-}\right) U\left(\mathcal{L}_{0}\right) U\left(A_{1}\right) \hat{N} \\
& =U\left(\mathcal{L}_{-2}(J)\right) \hat{N}
\end{aligned}
$$

pois $\hat{N}$ é um $A$-submódulo e, portanto, podemos considerá-lo um $U(A)$-submódulo de $\hat{M}$.

Para $u \in M(\lambda)_{\mu}$, denotamos por $\omega(u)=\mu$. Então,

$$
\omega(U(\mathcal{L}(J)))=\left\{\mu+\psi \mid \mu \in \omega\left(U\left(\mathcal{L}_{-2}\right) \backslash\{0\}\right), \psi \in \omega(\hat{N})\right\} \cup \omega(\hat{N}) .
$$

Mas $\lambda \neq \omega(U(\mathcal{L}(J)) \hat{N})$ e portanto $U(\mathcal{L}(J)) \hat{N}$ é um $\mathcal{L}(J)$-módulo próprio de $M(\lambda)$.

Denotamos por $N(\lambda)$ o único submódulo maximal próprio de $M(\lambda)$.

Lema 3.4.5. Se $\hat{M}$ é um A-módulo irredutível, então $N(\lambda)^{\left(\lambda\left(\mathbf{d}_{2}\right)\right)}=\{0\}$.

Prova: Como $M(\lambda)=U\left(\mathcal{L}_{-}\right) v_{\lambda}$ temos

$$
M(\lambda)^{\left(\lambda\left(\mathbf{d}_{2}\right)\right)}=U\left(A_{-}\right) v_{\lambda}=\hat{M} .
$$

Então

$$
N(\lambda)^{\left(\lambda\left(\mathbf{d}_{\mathbf{2}}\right)\right)} \subset M(\lambda)^{\left(\lambda\left(\mathbf{d}_{\mathbf{2}}\right)\right)} \cap N(\lambda)=\hat{M} \cap N(\lambda) .
$$


Como $\hat{M}$ é irredutível e $\hat{M} \cap N(\lambda)$ é um submódulo de $\hat{M}$ temos $\hat{M} \cap N(\lambda)=\{0\}$ ou $\hat{M} \cap N(\lambda)=\hat{M}$. Se $\hat{M} \cap N(\lambda)=\hat{M}$ então $1 \otimes v_{\lambda} \in N(\lambda)$, o que é uma contradição, pois $N(\lambda)$ é um submódulo próprio de $M(\lambda)$. Portanto, $N(\lambda)^{\left(\lambda\left(\mathbf{d}_{2}\right)\right)}=\{0\}$.

Lema 3.4.6. Se $N(\lambda)^{\left(-n_{0}+\lambda\left(\mathbf{d}_{\mathbf{2}}\right)\right)} \neq\{0\}$, para algum $n_{0} \in \mathbb{Z}$ e $n_{0}>0$, então $N(\lambda)^{\left(-n_{0}+n+\lambda\left(\mathbf{d}_{2}\right)\right)} \neq$ $\{0\}$, para algum $n \in \mathbb{Z}$ tal que $0<n \leq n_{0}$.

Prova: Seja $u \in N(\lambda)^{\left(-n_{0}+\lambda\left(\mathbf{d}_{2}\right)\right)}, u \neq 0$ e

$$
u=\sum u_{i, m, n, p} x_{i, m, n, p} v_{\lambda},
$$

onde $u_{i, m, n, p} \in \mathbb{C}$. Denotamos por $X$ o conjunto finito de todos os índices $(i, m, n, p)$ com $u_{i, m, n, p} \neq 0$.

Usando a ordem total da base $U\left(\mathcal{L}_{-}(J)\right)$ definida ns seção 3.3 obtemos um único $(a, b, c, s) \in X \operatorname{com} x_{a, b, c, s}$ maximal entre os monômios $x_{i, m, n, p} \operatorname{com}(i, m, n, p) \in X$.

Denotamos por $a=\left(a_{1}, \cdots, a_{\xi}\right), b=\left(b_{1}, \cdots, b_{\xi}\right), c=\left(c_{1}, \cdots, c_{\xi}\right), s=\left(s_{1}, \cdots, s_{\xi}\right)$. Desde que $n_{0}>0$ nem todos os $c_{i}^{\prime} s$ são nulos. Fixamos $r$ tal que $c=\left(c_{1}, \cdots, c_{r}, 0, \cdots, 0\right)$ e $c_{i}<0$ para $1 \leq i \leq r$. Se $c_{i} \neq c_{j}$ para algum $j<r$ seja $k$ tal que $c_{k-1}<c_{k}=c_{k+1}=$ $\cdots=c_{r}$ e, caso contrário, seja $k=1$.

Sejam

$$
\begin{aligned}
\bar{x} & =x_{a_{1}}\left(b_{1}, c_{1}\right)^{s_{1}} \cdots x_{a_{k-1}}\left(b_{k-1}, c_{k-1}\right)^{s_{k-1}}, \\
\tilde{x} & =x_{a_{k}}\left(b_{k}, c_{r}\right)^{s_{k}} \cdots x_{a_{r}}\left(b_{r}, c_{r}\right)^{s_{r}} \mathrm{e} \\
x^{\prime} & =x_{a_{k}+1}\left(b_{k+1}, 0\right)^{s_{k+1}} \cdots x_{a_{\xi}}\left(b_{\xi}, 0\right)^{s_{\xi}}
\end{aligned}
$$

então $x_{a, b, c, s}=\bar{x} \tilde{x} x^{\prime}$.

Seja $I=\left\{i_{j} \mid i=\left(i_{1}, \cdots, i_{j}, \cdots\right)\right.$, para $\left.(i, m, n, p) \in X\right\}$ o conjunto finito de todos os $i_{j}$ que aparecem em algum $i \operatorname{com}(i, m, n, p)$ pertencente a $X$.

Usando a definição de $r$ acima definimos o seguinte elemento

$$
y=s_{r} u_{a, b, c, s} x_{a_{r}}\left(b_{r}, 0\right) .
$$

Como $0 \neq y \in\left(s l_{2}(\mathbb{C}) \otimes J\right) \cap A$, pelo Lema 3.4 .3 existe um elemento $z(2 K, 0) \in\left(s l_{2}(\mathbb{C}) \otimes\right.$ $J) \cap A_{\beta}$ para alguma raiz $\beta$ de $A$ tal que

$$
0 \neq[z(2 K, 0), y], \quad[z(2 K,-l)], y(l)]=[z(2 K, 0), y]
$$


e

$$
\left[z(2 K,-l), x_{i_{j}}\left(m_{j}, l\right)\right]=\left[z(2 K, 0), x_{i_{j}}\left(m_{j}, 0\right)\right] \text { ou } 2 x_{i_{j}}\left(2 K+m_{j}, 0\right),
$$

para todo $l \in \mathbb{Z}$ e $i_{j} \in I$.

Consideramos a ação de $z\left(2 K,-c_{r}\right)$ sobre o elemento $u$. Provaremos que $z\left(2 K,-c_{r}\right) u \neq$ 0 e como $z\left(2 K,-c_{r}\right) u$ está contido em $N(\lambda)^{\left(-n_{0}-c_{r}+\lambda\left(\mathbf{d}_{\mathbf{2}}\right)\right)}$ e $0<-c_{r} \leq n_{0}$ o lema se verifica.

Primeiramente analisemos a ação de $z\left(2 K,-c_{r}\right)$ sobre o termo $u_{a, b, c, s} x_{a, b, c, s} v_{\lambda}$ de $u$ :

$$
z\left(2 K,-c_{r}\right) u_{a, b, c, s} x_{a, b, c, s} v_{\lambda}=u_{a, b, c, s}\left[z\left(2 K,-c_{r}\right), \bar{x}\right] \tilde{x} x^{\prime} v_{\lambda}+u_{a, b, c, s} \bar{x}\left[z\left(2 K,-c_{r}\right), \tilde{x}\right] x^{\prime} v_{\lambda}
$$

a igualdade se verifica como $-c_{r}>0$ e, portanto, a ação de $z\left(2 K,-c_{r}\right)$ sobre $x^{\prime} v_{\lambda}$ é trivial.

Pelo Lema 3.4.1 o elemento $\left[z\left(2 K,-c_{r}\right), \bar{x}\right]$ está contido em $U\left(\mathcal{L}_{-2}(J)\right) \mathcal{L}_{-2}(J)$ e como $\tilde{x}$ também está em $U\left(\mathcal{L}_{-2}(J)\right) \mathcal{L}_{-2}(J)$, denotaremos por:

$$
y_{1}:=u_{a, b, c, s}\left[z\left(2 K,-c_{r}\right), \bar{x}\right] \tilde{x} .
$$

Observamos que, pelo Lema 3.4 .2 existe um elemento $y_{2} \in U\left(\mathcal{L}_{-2}(J)\right) \mathcal{L}_{-2}(J)$ tal que

$$
\left[z\left(2 K,-c_{r}\right), \tilde{x}\right]=y_{2}+\sum_{j=k}^{r} s_{j} \hat{x}^{\hat{s_{j}}}\left[z\left(2 K,-c_{r}\right), x_{a_{j}}\left(b_{j}, c_{r}\right)\right] .
$$

Então

$$
\begin{aligned}
z\left(2 K,-c_{r}\right) u_{a, b, c, s} x_{a, b, c, s} v_{\lambda}= & y_{1} x^{\prime} v_{\lambda}+u_{a, b, c, s} \bar{x} y_{2} x^{\prime} v_{\lambda} \\
& +u_{a, b, c, s} \sum_{j=k}^{r} s_{j} \bar{x} \tilde{x}^{\hat{s_{j}}}\left[z\left(2 K,-c_{r}\right), x_{a_{j}}\left(b_{j}, c_{r}\right)\right] x^{\prime} v_{\lambda} \\
= & \left(y_{1}+u_{a, b, c, s} \bar{x} y_{2}\right) x^{\prime} v_{\lambda} \\
& +u_{a, b, c, s} \sum_{j=k}^{r-1} s_{j} \bar{x} \tilde{x}^{\hat{s_{j}}}\left[z\left(2 K,-c_{r}\right), x_{a_{j}}\left(b_{j}, c_{r}\right)\right] x^{\prime} v_{\lambda} \\
& +u_{a, b, c, s} s_{r} \bar{x} \tilde{x}^{\hat{s_{r}}}\left[z\left(2 K,-c_{r}\right), x_{a_{j}}\left(b_{j}, c_{r}\right)\right] x^{\prime} v_{\lambda} .
\end{aligned}
$$

Denotamos por

$$
\begin{aligned}
& T_{1}:=\left(y_{1}+u_{a, b, c, s} \bar{x} y_{2}\right) x^{\prime} v_{\lambda} \\
& T_{2}:=u_{a, b, c, s} \sum_{j=k}^{r-1} s_{j} \bar{x} \tilde{x}^{\hat{s_{j}}}\left[z\left(2 K,-c_{r}\right), x_{a_{j}}\left(b_{j}, c_{r}\right)\right] x^{\prime} v_{\lambda} \\
& T_{3}:=u_{a, b, c, s} s_{r} \bar{x} \tilde{x}^{\hat{s_{r}}}\left[z\left(2 K,-c_{r}\right), x_{a_{r}}\left(b_{r}, c_{r}\right)\right] x^{\prime} v_{\lambda} .
\end{aligned}
$$


Pela escolha do elemento $z(2 K, 0)$ o termo $T_{3}$ é não-nulo. Como $y_{1}+u_{a, b, c, s} \bar{x} y_{2} \in$ $U\left(\mathcal{L}_{-2}(J)\right) \mathcal{L}_{-2}(J)$ e, pela condição 2 do Lema 3.4 .3 , o termo $T_{1}$ não pode contribuir para anular $T_{3}$. Nos monômios que aparecem em $T_{2}$ nenhum possui potências não-nulas de $x_{a_{r}}\left(b_{r}, c_{r}\right)$ e, portanto, $T_{2}$ não pode anular $T_{3}$.

Consideramos um somando arbitrário não-nulo $u_{i, m, n, p} x_{i, m, n, p} v_{\lambda}$ de $u v_{\lambda}$ tal que $(i, m, n, p) \neq$ $(a, b, c, s)$.

$$
x_{i, m, n, p}=x_{i_{1}}\left(m_{1}, n_{1}\right)^{p_{1}} \cdots x_{i_{q}}\left(m_{q}, n_{q}\right)^{p_{q}} x_{i_{q+1}}\left(m_{q+1}, 0\right)^{p_{q+1}} \cdots x_{i_{\epsilon}}\left(m_{\epsilon}, 0\right)^{p_{\epsilon}} .
$$

Expandiremos $z\left(2 K,-c_{r}\right) u_{i, m, n, p} x_{i, m, n, p} v_{\lambda}$ e verificaremos que esse termo não contribui para cancelar $T_{3}$.

Se $n_{q}<c_{r}$ então, pelo Lema 3.4 .1 e o fato que $z\left(2 K,-c_{r}\right) U\left(\mathcal{L}_{-1}(J)\right) v_{\lambda}=0$, temos que

$$
z\left(2 K,-c_{r}\right) u_{i, m, n, p} x_{i, m, n, p} v_{\lambda}=y^{\prime} x_{i_{q+1}}\left(m_{q+1}, 0\right)^{p_{q+1}} \cdots x_{i_{\epsilon}}\left(m_{\epsilon}, 0\right)^{p_{\epsilon}} v_{\lambda}
$$

para algum $y^{\prime} \in U\left(\mathcal{L}_{-2}(J)\right) \mathcal{L}_{-2}(J)$.

Pela condição 2 do Lema 3.4.3, o somando acima não pode contribuir para cancelar $T_{3}$.

Se $n_{q}=c_{r}$, então pela definição da ordem total sobre a base de $U(\mathcal{L}(J))$ e pela maximalidade de $(a, b, c, s)$ em $X$, temos que $n_{1} \leq n_{2} \leq \cdots \leq n_{q}=c_{r}$. Assim podemos supor que $n_{\gamma}<n_{\gamma+1}=\cdots=n_{q}=c_{r}$. Denotamos por

$$
\begin{aligned}
\bar{w} & =x_{i_{1}}\left(m_{1}, n_{1}\right)^{p_{1}} \cdots x_{i_{\gamma-1}}\left(m_{\gamma-1}, n_{\gamma-1}\right)^{p_{\gamma-1}} \\
\tilde{w} & =x_{i_{\gamma}}\left(m_{\gamma}, c_{r}\right)^{p_{\gamma}} \cdots x_{i_{q}}\left(m_{q}, c_{r}\right)^{p_{q}} \\
w^{\prime} & =x_{i_{q+1}}\left(m_{q+1}, 0\right)^{p_{q+1}} \cdots x_{i_{\epsilon}}\left(m_{\epsilon}, 0\right)^{p_{\epsilon}}
\end{aligned}
$$

Novamente pelos Lemas 3.4.1 e 3.4.2 existe um $y^{\prime} \in U\left(\mathcal{L}_{-2}(J)\right) \mathcal{L}_{-2}(J)$ tal que

$$
z\left(2 K,-c_{r}\right) x_{i, m, n, p} v_{\lambda}=y^{\prime} w^{\prime} v_{\lambda}+\sum_{j=\gamma}^{q} p_{j} \bar{w} \tilde{w}^{\hat{p_{j}}}\left[z\left(2 K,-c_{r}\right), x_{i_{j}}\left(m_{j}, c_{r}\right)\right] w^{\prime} v_{\lambda}
$$

Denotamos por

$$
\begin{aligned}
& S_{1}=y^{\prime} w^{\prime} v_{\lambda} \\
& S_{2}=\sum_{j=\gamma}^{q} p_{j} \bar{w} \tilde{w}^{\hat{p_{j}}}\left[z\left(2 K,-c_{r}\right), x_{i_{j}}\left(m_{j}, c_{r}\right)\right] w^{\prime} v_{\lambda} .
\end{aligned}
$$


Observamos que $S_{1}$ não contribuirá para cancelar $T_{3}$ devido à condição 2 da escolha de $z$ e pelo fato de $y^{\prime}$ pertencer a $U\left(\mathcal{L}_{-2}(J)\right) \mathcal{L}_{-2}(J)$.

Caso 1: $s_{r} \geq 2$.

Supondo que $S_{2}$ contribua para cancelar $T_{3}$ teríamos

$$
\begin{aligned}
\bar{x} x_{a_{k}} & \left(b_{k}, c_{r}\right)^{s_{k}} \cdots x_{a_{r-1}}\left(b_{r-1}, c_{r}\right)^{s_{r-1}} x_{a_{r}}\left(b_{r}, c_{r}\right)^{s_{r}-1} \\
& =\bar{w} x_{i_{\gamma}}\left(m_{\gamma}, c_{r}\right)^{p_{\gamma}} \cdots x_{i_{j}}\left(m_{j}, c_{r}\right)^{p_{j}-1} \cdots x_{i_{q}}\left(m_{q}, c_{r}\right)^{p_{q}}
\end{aligned}
$$

para algum $j$. Como $\bar{x}$ e $\bar{w}$ são monômios em $x_{i_{l}}\left(m_{l}, n\right)^{\prime} s, \operatorname{com} n<c_{r}$, temos $\bar{x}=\bar{w}$ e

$$
\begin{aligned}
& x_{a_{k}}\left(b_{k}, c_{r}\right)^{s_{k}} \cdots x_{a_{r-1}}\left(b_{r-1}, c_{r}\right)^{s_{r-1}} x_{a_{r}}\left(b_{r}, c_{r}\right)^{s_{r}-1} \\
& \quad=x_{i_{\gamma}}\left(m_{\gamma}, c_{r}\right)^{p_{\gamma}} \cdots x_{i_{j}}\left(m_{j}, c_{r}\right)^{p_{j}-1} \cdots x_{i_{q}}\left(m_{q}, c_{r}\right)^{p_{q}}
\end{aligned}
$$

Pela maximalidade de $(a, b, c, s)$ precisamos ter $j=q$, caso contrário o monômio

$$
x_{i_{\gamma}}\left(m_{\gamma}, c_{r}\right)^{p_{\gamma}} \cdots x_{i_{j}}\left(m_{j}, c_{r}\right)^{p_{j}-1} \cdots x_{i_{q}}\left(m_{q}, c_{r}\right)^{p_{q}}
$$

seria um monômio com $p_{q}=s_{r}-1<s_{r}$ e, por hipótese, $s_{r}-1>0$. Portanto, isso implicaria que o termo $x_{i, m, n, p}$ seria maior do que $x_{a, b, c, s}$, contradizendo a maximalidade de $x_{a, b, c, s}$. Logo $j=q$ e, portanto, $(i, m, n, p)=(a, b, c, s)$, contradição.

Caso 2: $s_{r}=1$.

Suponha que $S_{2}$ contribua para cancelar $T_{3}$. Neste caso, temos

$$
\begin{aligned}
\bar{x} x_{a_{k}}\left(b_{k}, c_{r}\right)^{s_{k}} & \cdots x_{a_{r-1}}\left(b_{r-1}, c_{r}\right)^{s_{r-1}} \\
& =\bar{w} x_{i_{\gamma}}\left(m_{\gamma}, c_{r}\right)^{p_{\gamma}} \cdots x_{i_{j}}\left(m_{j}, c_{r}\right)^{p_{j}-1} \cdots x_{i_{q}}\left(m_{q}, c_{r}\right)^{p_{q}}
\end{aligned}
$$

para $\operatorname{algum} j$.

Pelo mesmo argumento do caso $s_{r} \geq 2$, temos $\bar{x}=\bar{w}$ e

$$
\begin{aligned}
x_{a_{k}}\left(b_{k}, c_{r}\right)^{s_{k}} & \cdots x_{a_{r-1}}\left(b_{r-1}, c_{r}\right)^{s_{r-1}} \\
& =x_{i_{\gamma}}\left(m_{\gamma}, c_{r}\right)^{p_{\gamma}} \cdots x_{i_{j}}\left(m_{j}, c_{r}\right)^{p_{j}-1} \cdots x_{i_{q}}\left(m_{q}, c_{r}\right)^{p_{q}}
\end{aligned}
$$

Pela igualdade acima podemos concluir que $x_{i_{j}}\left(m_{j}, 0\right) \leq x_{a_{r}}\left(b_{r}, 0\right)$ e que o elemento $\left[z\left(2 K,-c_{r}\right), x_{i_{q}}\left(m_{q}, c_{r}\right)\right] w^{\prime}$ pertence ao subespaço $\mathbb{C}\left[z(2 K, 0), x_{a_{r}}\left(b_{r}, 0\right)\right] x^{\prime}$. Supondo que $x_{i_{j}}\left(m_{j}, 0\right)<x_{a_{r}}\left(b_{r}, 0\right)$, isto é,

$$
\text { ou } m_{j}<b_{r} \text { ou } m_{j}=b_{r} \text { e } \beta_{i_{j}}<\beta_{a_{r}}
$$


onde $\beta_{i_{j}}$ e $\beta_{a_{r}}$ são as raízes associadas aos elementos $x_{i_{j}}$ e $x_{a_{r}}$, respectivamente. As$\operatorname{sim}\left[z\left(2 K,-c_{r}\right), x_{i_{q}}\left(m_{q}, c_{r}\right)\right] w^{\prime}$ teria o menor termo em $A_{\beta+\beta_{i_{j}}+\left(2 K+m_{q}\right) \delta_{1}}$ enquanto que $\left[z(2 K, 0), x_{a_{r}}\left(b_{r}, 0\right)\right] x^{\prime}$ teria o menor termo em $A_{\beta+\beta_{a_{r}}+\left(2 K+b_{r}\right) \delta_{1}}$. Isto é impossível se eles se cancelam. Portanto, $x_{i_{j}}\left(m_{j}, 0\right)=x_{a_{r}}\left(b_{r}, 0\right)$ e isto implica que

$$
\begin{aligned}
\bar{w} x_{i_{\gamma}} & \left(m_{\gamma}, c_{r}\right)^{p_{\gamma}} \cdots x_{i_{q}}\left(m_{q}, c_{r}\right)^{p_{q}} w^{\prime} \\
& =\bar{w} x_{a_{k}}\left(b_{k}, c_{r}\right)^{s_{k}} \cdots x_{a_{r-1}}\left(b_{r-1}, c_{r}\right)^{s_{r-1}} x_{i_{j}}\left(m_{j}, c_{r}\right) w^{\prime} \\
& =\bar{x} x_{a_{k}}\left(b_{k}, c_{r}\right)^{s_{k}} \cdots x_{a_{r-1}}\left(b_{r-1}, c_{r}\right)^{s_{r-1}} x_{a_{r}}\left(b_{r}, c_{r}\right) x^{\prime}
\end{aligned}
$$

ou seja, $(i, m, n, p)=(a, b, c, s)$, contradição. Assim provamos o lema.

Os resultados a seguir são análogos aos obtidos em [4] para o caso toroidal. Alteramos as demonstrações apropriadamente para o nosso caso.

Lema 3.4.7. Seja $N$ um submódulo não-nulo de $M(\lambda)$. Seja $0 \neq u v_{\lambda} \in N$ tal que

$$
u=\sum_{i \in I} x_{\beta_{i}}\left(-m_{i}, 0\right) u_{i}
$$

onde $0 \neq x_{\beta_{i}}\left(-m_{i}, 0\right) \in A_{\beta_{i}-m_{i} \delta_{1}}$ são linearmente independentes, $m_{i}>0$ e $u_{i} \in U\left(\mathcal{L}_{-1}(J)\right)$. Se $\max _{i \in I}\left\{\left[-\frac{m_{i}}{2}\right]\right\}<\min _{i \in I}\left\{\operatorname{deg}_{1} u_{i}\right\}$ e $\lambda\left(\mathbf{c}_{\mathbf{1}}\right) \neq 0$, então $u_{i} v_{\lambda} \in N$, para todo $i$.

Prova: Provaremos por indução sobre $|I|$. Suponhamos que $|I|=1$, então

$$
u=x_{\beta}(-m, 0) u^{\prime}
$$

Se $\beta=0$, temos

$$
\begin{aligned}
x_{\beta}(m, 0) x_{\beta}(-m, 0) u^{\prime} v_{\lambda} & =\left[x_{\beta}(m, 0), x_{\beta}(-m, 0)\right] u^{\prime} v_{\lambda}+x_{\beta}(-m, 0) x_{\beta}(m, 0) u^{\prime} v_{\lambda} \\
& =4 m \mathbf{c}_{\mathbf{1}} u^{\prime} v_{\lambda}=4 m \lambda\left(\mathbf{c}_{\mathbf{1}}\right) u^{\prime} v_{\lambda},
\end{aligned}
$$

pois $m+\operatorname{deg}_{1} u^{\prime} \geq\left[\frac{m}{2}\right]+\operatorname{deg}_{1} u^{\prime}>0$ implica $x_{\beta}(m, 0) u^{\prime} v_{\lambda}=0$. Como $\lambda\left(\mathbf{c}_{\boldsymbol{1}}\right) \neq 0$, temos $u^{\prime} v_{\lambda} \in N$. Se $\beta \neq 0$, temos

$$
\begin{aligned}
{\left[x_{-\beta}([m / 2], 0), x_{\beta}(-m, 0)\right] u^{\prime} v_{\lambda} } & =x_{-\beta}([m / 2], 0) x(-m, 0) u^{\prime} v_{\lambda}-x_{\beta}(-m, 0) x_{-\beta}([m / 2], 0) u^{\prime} v_{\lambda} \\
& =x_{-\beta}([m / 2], 0) x_{\beta}(-m, 0) u^{\prime} v_{\lambda}
\end{aligned}
$$

pois $\left[\frac{m}{2}\right]+\operatorname{deg}_{1} u^{\prime}>0$ e, portanto, $x_{-\beta}\left(\left[\frac{m}{2}\right], 0\right) u^{\prime} v_{\lambda}=0$. Utilizando o argumento anterior para $\left[x_{-\beta}\left(\left[\frac{m}{2}\right], 0\right), x_{\beta}(-m, 0)\right] u^{\prime} v_{\lambda}$, verificamos o lema para $|I|=1$. 
Supomos agora que $|I|>1$ e assumimos que o lema se verifica para elementos com um número menor do que $|I|$ somandos.

Seja $m=\min _{i \in I}\left\{m_{i}\right\}$ e seja $I^{\prime} \subset I$ o subconjunto de todos os $j \in I$ tais que $m_{j}=m$.

Supondo que existe um $j \in I$ tal que $\beta_{j} \neq 0$. Consideramos o caso em que exista pelo menos um $k \in I^{\prime}$ tal que $\beta_{k}=0$. Então consideramos o elemento $x_{\beta_{k}}\left(\left[\frac{m}{2}\right], 0\right)$ tal que $\left[x_{\beta_{k}}\left(\left[\frac{m}{2}\right], 0\right), x_{\beta_{j}}(-m, 0)\right] \neq 0$. Aplicando $x_{\beta_{k}}\left(\left[\frac{m}{2}\right], 0\right)$ a $u v_{\lambda}$ obtemos

$$
x_{\beta_{k}}([m / 2], 0) u v_{\lambda}=\sum_{i \in I^{\prime}}\left[x_{\beta_{k}}([m / 2], 0), x_{\beta_{i}}(-m, 0)\right] u_{i} v_{\lambda}+\sum_{i \notin I^{\prime}}\left[x_{\beta_{k}}([m / 2], 0), x_{\beta_{i}}\left(-m_{i}, 0\right)\right],
$$

como $\left[\frac{m}{2}\right]+\operatorname{deg}_{1} u_{i}>0$ temos $x_{\beta_{k}}\left(\left[\frac{m}{2}\right], 0\right) u_{i} v_{\lambda}=0$.

Os somandos com índices $k \in I^{\prime}$ tal que $\beta_{k}=0$ são nulos pois $\left[x_{\beta_{k}}\left(\left[\frac{m}{2}\right], 0\right), x_{\beta_{k}}(-m, 0)\right]=$ 0 . Para os índices $j \in I$ com $\beta_{j} \neq 0$, temos $\left[x_{\beta_{k}}\left(\frac{m}{2}, 0\right), x_{\beta_{j}}\left(-m_{j}, 0\right)\right] \neq 0$. Assim assumindo a existência de $k \in I^{\prime}$, com $\beta_{k}=0$, o número de somandos em $x_{\beta_{k}}([m / 2], 0) u v_{\lambda}$ é menor do que o número de somandos em $u v_{\lambda}$. Observamos que, para $m>>0$ os somandos em $x_{\beta_{k}}([m / 2], 0) u v_{\lambda}$ são linearmente independentes e, portanto, $x_{\beta_{k}}([m / 2], 0) u v_{\lambda} \neq 0$.

Pela hipótese de indução, $u_{j} v_{\lambda} \in N$ o que implica $\left(u-x_{\beta_{j}}\left(m_{j}, 0\right)\right) v_{\lambda} \in N$. Assim aplicamos a hipótese de indução sobre $\left(u-x_{\beta_{j}}\left(m_{j}, 0\right)\right) v_{\lambda}$ e obtemos $u_{i} v_{\lambda} \in N$, para todo $i \in I$ quando existem índices $k \in I^{\prime}$ tais que $\beta_{k}=0$.

Supomos que $\beta_{k} \neq 0$ para todo $k \in I^{\prime}$ e fixamos um $k_{0} \in I^{\prime}$. Consideramos um elemento não-nulo $x_{-\beta_{k_{0}}}\left(\left[\frac{m}{2}\right], 0\right)$ e aplicamos a $u v_{\lambda}$. Como $m>>0$, os somandos em $x_{-\beta_{k_{0}}}\left(\left[\frac{m}{2}\right], 0\right) u v_{\lambda}$ são linearmente independentes e, portanto, $x_{-\beta_{k_{0}}}\left(\left[\frac{m}{2}\right], 0\right) u v_{\lambda} \neq 0$. Esse elemento contém o somando $\left[x_{-\beta_{k_{0}}}\left(\left[\frac{m}{2}\right], 0\right), x_{\beta_{k_{0}}}(m, 0)\right] u_{k_{0}} v_{\lambda}$ e o número de somandos em $x_{-\beta_{k_{0}}}\left(\left[\frac{m}{2}\right], 0\right) u v_{\lambda}$ não aumenta. Logo podemos aplicar o argumento anterior a $x_{-\beta_{k_{0}}}\left(\left[\frac{m}{2}\right], 0\right) u v_{\lambda}$ e obter o resultado desejado para este caso.

Por último consideramos o caso em que $\beta_{i}=0$, para todo $i \in I$. Fixamos um $i_{0} \in I^{\prime}$ e consideramos o elemento $x_{\beta_{i_{0}}}(-m, 0)$ aplicado a $u v_{\lambda}$. Obsevamos que

$$
\left[x_{\beta_{i_{0}}}(m, 0), x_{\beta_{j}}\left(m_{j}, 0\right)\right]=0
$$

para todo $j \neq i_{0}$. Assim, para $\lambda\left(\mathbf{c}_{\mathbf{1}}\right) \neq 0$ obtemos $u_{i_{0}} v_{\lambda} \in N$ e portanto $\left(u-u_{i_{0}}\right) v_{\lambda} \in N$. Agora aplicando a hipótese de indução sobre o elemento $\left(u-u_{i_{0}}\right) v_{\lambda}$ provamos o lema. 
Lema 3.4.8. Seja $N$ um submódulo de $M(\lambda)$. Suponha que $\mathbf{c}_{\mathbf{1}}$ age por multiplicação por escalar não-nulo sobre $M(\lambda)$. Sejam $m, n \in \mathbb{Z}, n>0$. Se $0 \neq u v_{\lambda} \in N$, com

$$
u=\sum_{i \in I} h_{i}(m,-n) u_{i}
$$

onde $u_{i} \in U\left(\mathcal{L}_{-1}(J)\right)$ são elementos linearmente independentes e $h_{i}(m,-n)=h \otimes x^{(m,-n)}$, se $(m,-n) \in S$ ou $2\left[L_{x^{\delta_{1}}}, L_{x^{(m,-n)-\delta_{1}}}\right]$, se $(m,-n) \in S^{\perp}$, então $u_{i} v_{\lambda} \in N$, para todo $i \in I$.

Prova: Provaremos por indução sobre $|I|$. Supondo que $|I|=1$, temos

$$
u=h(m,-n) u^{\prime}
$$

Consideremos o elemento $x_{\beta}(-2 M, n)$ tal que $\beta \neq 0$ e $M>>0$. Então,

$$
\left[x_{\beta}(-2 M, n), h(m,-n)\right] \neq 0
$$

e

$$
\begin{aligned}
0 \neq x_{\beta}(-2 M, n) u v_{\lambda} & =\left[x_{\beta}(-2 M, n), h(m,-n)\right] u^{\prime} v_{\lambda} \\
& =-\beta(h) x_{\beta}(-2 M+m, 0) u^{\prime} v_{\lambda}
\end{aligned}
$$

Utilizando o fato que $\lambda\left(\mathbf{c}_{\mathbf{1}}\right) \neq 0$, podemos aplicar o Lema 3.4 .7 ao elemento $x_{\beta}(-2 M, n) u v_{\lambda}$. Assim obtemos $u^{\prime} v_{\lambda} \in N$.

Suponha agora $|I|>1$. Assumimos que o lema se verifica para um número menor do que $|I|$ somandos. Fixamos um $i_{0} \in I$. Escolhemos um elemento não-nulo $x_{\beta}(-2 M, n)$ $\operatorname{com} \beta \neq 0$ e $M>>0$ tal que $\left[x_{\beta}(-2 M, n), h_{i_{0}}(m,-n)\right] \neq 0$. Então, pelo fato do $u_{i}$ serem linearmente independentes e $M>>0$, temos

$$
\begin{aligned}
0 \neq x_{\beta}(-2 M, n) u v_{\lambda} & =\sum_{i \in I}\left[x_{\beta}(-2 M, n), h_{i}(m,-n)\right] u_{i} v_{\lambda} \\
& =-\sum_{i \in I} \beta\left(h_{i}\right) x_{\beta}(-2 M+m, 0) u_{i} v_{\lambda} \\
& =\sum_{i \in I} a_{i} u_{i} v_{\lambda},
\end{aligned}
$$

onde $a_{i}=-\beta\left(h_{i}\right) x_{\beta}(-2 M+m, 0)$. Observamos que $a_{i}=k_{i} a_{i_{0}}$, para $k_{i} \in \mathbb{C}$ e $i \in I, \operatorname{logo}$

$$
\sum_{i \in I} a_{i} u_{i} v \lambda=a_{i_{0}} \sum_{i \in I} k_{i} u_{i} v_{\lambda}
$$


Como $\lambda\left(\mathbf{c}_{\mathbf{1}}\right) \neq 0$, podemos aplicar o Lema 3.4.7 no elemento $a_{i_{0}} \sum_{i \in I} k_{i} u_{i} v_{\lambda}$ e obtemos $\sum_{i \in I} k_{i} u_{i} v_{\lambda} \in N$. Logo,

$$
\left(u-\frac{1}{k_{i_{0}}} h_{i_{0}}(m,-n) \sum_{i \in I} k_{i} u_{i}\right) v_{\lambda} \in N
$$

e

$$
\left(u-\frac{1}{k_{i_{0}}} h_{i_{0}}(m,-n) \sum_{i \in I} k_{i} u_{i}\right) v_{\lambda}=\sum_{i \in I, i \neq i_{0}}\left(h_{i}(m,-n)-\frac{k_{i}}{i_{0}} h_{i_{0}}(m,-n)\right) u_{i} v_{\lambda} .
$$

Aplicando a hipótese de indução sobre o elemento acima provamos o lema.

Agora para $N \subset M(\lambda)$ denotamos por $\hat{N}=N \cap \hat{M}$.

Teorema 3.4.9. Se $\lambda\left(\mathbf{c}_{\boldsymbol{1}}\right) \neq 0$ e $N \subset M(\lambda)$ então $N \cong U\left(\mathcal{L}_{-2}(J)\right) \otimes_{\mathbb{C}} \hat{N}$.

Prova: Seja $0 \neq u v_{\lambda} \in N \operatorname{com} u \in U\left(\mathcal{L}_{-}(J)\right)$. Pela Proposição 1 de [14] Seção 2.1 podemos assumir que $u$ é um elemento homogêneo e pelo Teorema de PBW podemos escrever $u$ da seguintes forma

$$
u=\sum_{i \in I} u_{i} u_{i}^{\prime}
$$

onde $u_{i} \in U\left(\mathcal{L}_{-2}(J)\right)$ são linearmente independentes e $u_{i}^{\prime} \in U\left(\mathcal{L}_{-1}(J)\right)$ com

$$
u_{i}=x_{\beta_{1_{i}}}\left(m_{1_{i}},-n_{1_{i}}\right)^{p_{1_{i}}} \cdots x_{\beta_{s_{i}}}\left(m_{s_{i}},-n_{s_{i}}\right)^{p_{s_{i}}}
$$

$\operatorname{com} m_{j_{i}}, n_{j_{i}} \in \mathbb{Z}, n_{j_{i}}>0, x_{\beta_{j_{i}}} \in B_{2}$ e $x_{\beta_{1_{i}}}\left(m_{1_{i}},-n_{1_{i}}\right)<\cdots<x_{\beta_{s_{i}}}\left(m_{s_{i}},-n_{s_{i}}\right)$. Queremos provar que $u_{i}^{\prime} v_{\lambda} \in N$, pois $u_{i}^{\prime} v_{\lambda} \in \hat{M}$. Provaremos este resultado por indução sobre $\operatorname{deg}_{2} u$ e a ordem de $I$.

Suponha que $\operatorname{deg}_{2} u=-1$ e $|I|=1$. Então

$$
u=x_{\beta}(m,-1) u^{\prime}
$$

Se $\beta=0$ então o resultado segue do Lema 3.4.8. Se $\beta \neq 0$ consideramos o elemento $x_{-\beta}(-2 M, 1)$ com $2 M>>0$. Daí $\left[x_{-\beta}(-2 M, 1), x_{\beta}(m,-1)\right] \neq 0 \mathrm{e}$

$$
\begin{aligned}
x_{-\beta}(-2 M, 1) u v_{\lambda} & =\left[x_{-\beta}(-2 M, 1), x_{\beta}(m,-1)\right] u^{\prime} v_{\lambda}+x_{\beta}(m,-1) x_{-\beta}(-2 M, 1) u^{\prime} v_{\lambda} \\
& =\left[x_{-\beta}(-2 M, 1), x_{\beta}(m,-1)\right] u^{\prime} v_{\lambda} \neq 0,
\end{aligned}
$$

pois $x_{-\beta}(-2 M, 1) u^{\prime} v_{\lambda}=0$. Aplicando o Lema 3.4.8 ao elemento $x_{-\beta}(-2 M, 1) u v_{\lambda}$ obtemos o resultado desejado para este caso. 
Consideramos o caso $\operatorname{deg}_{2} u=-1$ e $|I|>1$. Assumimos que o teorema se verifica para um número menor do que $|I|$ somandos. Sejam $n=\min _{i \in I}\left\{n_{i_{s}}\right\}$ e $m=\min _{i \in I}\left\{m_{s_{i}} \mid\right.$ $\left.n_{s_{i}}=n\right\}$. Consideramos o subconjunto $I^{\prime}$ de $I$ consistindo $\operatorname{dos} i \in I$ tais que $n_{s_{i}}=n$ e $m_{s_{i}}=m$. Assim,

$$
u=\sum_{i \in I^{\prime}} x_{\beta_{i}}(m,-1) u_{i}+\sum_{i \notin I^{\prime}} x_{\beta_{i}} x\left(m_{i},-1\right) u_{i}
$$

Suponha que exista $j \in I$ tal que $\beta_{j} \neq 0$. Caso exista $l \in I^{\prime}$ com $\beta_{l}=0$ consideramos o elemento $x_{\beta_{l}}(-2 M, 1)$, para $M>>0$, tal que $\left[x_{\beta_{l}}(-2 M, 1), x_{\beta_{j}}\left(m_{j},-1\right)\right] \neq 0$ e $\left[x_{\beta_{l}}(-2 M, 1), x_{\beta_{l}}(m,-1)\right]=0$. Então

$$
\begin{aligned}
0 \neq x_{\beta_{l}}(-2 M, 1) u v_{\lambda} & =\sum_{i \in I^{\prime}}\left[x_{\beta_{l}}(-2 M, 1), x_{\beta_{i}}(m,-1)\right] u_{i} v_{\lambda} \\
& +\sum_{i \notin I^{\prime}}\left[x_{\beta_{l}}(-2 M, 1), x_{\beta_{i}}\left(m_{i},-1\right)\right] u_{i} v_{\lambda} .
\end{aligned}
$$

O elemento $x_{\beta_{l}}(-2 M, 1) u v_{\lambda}$ possui um número estritamente menor do que $|I|$ somandos. Aplicando a hipótese de indução sobre $x_{\beta_{l}}(-2 M, 1) u v_{\lambda}$ obtemos o resultado desejado.

Se $\beta_{i} \neq 0$, para todo $i \in I^{\prime}$, fixamos um elemento $i_{0} \in I^{\prime}$ e consideramos o elemento $x_{-\beta_{i_{0}}}(-2 M, 1)$, para $M>>0$, agindo sobre $u v_{\lambda}$. Como $M>>0$, os elementos $\left[x_{-\beta_{i_{0}}}(-2 M, 1), x_{\beta_{i}}\left(m_{i},-1\right)\right] \neq 0$, para $i \in I$, são linearmente independentes sobre $M(\lambda)$ e $\left[x_{-\beta_{i_{0}}}(-2 M, 1), x_{\beta_{i_{0}}}(m,-1)\right] \neq 0$ o que implica

$$
x_{-\beta_{i_{0}}}(-2 M, 1) u v_{\lambda} \neq 0
$$

Aplicando o Lema 3.4.7 ao elemento acima obtemos o resultado desejado.

Se $\beta_{i}=0$, para todo $i \in I$, consideramos o elemento $x_{\beta}(-2 M, 1) \operatorname{com} \beta \neq 0 \mathrm{e}$ $M>>0$. Então $\left[x_{\beta}(-2 M, 1), x_{\beta_{i}}\left(m_{i},-1\right)\right] \neq 0$, para todo $i \in I$, e como $M>>0$ os elementos $\left[x_{\beta}(-2 M, 1), x_{\beta_{i}}\left(m_{i},-1\right)\right]$ são linearmente independentes em $M(\lambda)$. Aplicando o Lema 3.4 .7 a $x_{\beta}(-2 M, 1) u v_{\lambda}$ verificamos o teorema para este caso.

Suponha que $\operatorname{deg}_{2} u<-1$ e $|I| \geq 1$. Como $u$ é homogêneo, $u v_{\lambda} \in N^{\left(-n_{0}+\lambda\left(\mathbf{d}_{2}\right)\right)}$ para algum $n_{0} \in \mathbb{Z}, n_{0}>0$. Na demonstração do Lema 3.4.6 encontramos um elemento $y$ tal que $0 \neq y u v_{\lambda} \in N^{\left(-n_{0}+n+\lambda\left(\mathbf{d}_{2}\right)\right)}$, para algum $n \in \mathbb{Z}$ tal que $0<n \leq n_{0}$. Como $\left|\operatorname{deg}_{2} y u v_{\lambda}\right|<\left|\operatorname{deg}_{2} u v_{\lambda}\right|$ podemos aplicar a hipótese de indução ao elemento yuv $v_{\lambda}$. O novo elemento $y u v_{\lambda}$ contém possivelemente mais somandos que $u v_{\lambda}$, mas um dos monômios não nulos contém $\left[z\left(2 K,-c_{r}\right), x_{a_{r}}\left(b_{r}, c_{r}\right)\right] x^{\prime} v_{\lambda}$ como vimos na demonstração do Lema 3.4.6, 
onde $x^{\prime}=u_{a}^{\prime}$, para $u_{a}$ maximal entre os $u_{i}^{\prime} s$. Portanto, $\left[z\left(2 K,-c_{r}\right), x_{a_{r}}\left(b_{r}, c_{r}\right)\right] x^{\prime} v_{\lambda} \in \hat{M}$. Como $K>>0$, pela condição de que $\lambda\left(\mathbf{c}_{1}\right) \neq 0$, podemos aplicar o Lema 3.4.6 ao elemento $\left[z\left(2 K,-c_{r}\right), x_{a_{r}}\left(b_{r}, c_{r}\right)\right] x^{\prime} v_{\lambda}$ e obter que $x^{\prime} v_{\lambda}=u_{a}^{\prime} v_{\lambda} \in \hat{M}$.

Agora aplicando a hipótese de induçao ao elemento $\left(u v_{\lambda}-k_{a} u_{a} u_{a}^{\prime}\right)$ obtemos o resultado desejado e encerramos a demonstração do teorema.

Teorema 3.4.10. $O \mathcal{L}(J)$-módulo $M(\lambda)$ é irredutível se, e somente se, $\hat{M}$ é um A-módulo irredutivel.

Prova: $(\Rightarrow)$ É o Lema 3.4.4.

$(\Leftarrow)$ Suponhamos que $M(\lambda)$ não seja irredutível. Seja $N(\lambda) \neq\{0\}$ seu submódulo maximal. Então $N(\lambda)^{\left(-n_{0}+\lambda\left(\mathbf{d}_{\mathbf{2}}\right)\right)}$, para algum $n_{0}>0$. Pelo Lema 3.4.6 $N(\lambda)^{\lambda\left(\mathbf{d}_{\mathbf{2}}\right)} \neq\{0\}$, mas $\hat{M}$ é irredutível e isso contradiz o Lema 3.4.5.

\subsection{Quociente integrável}

Mostraremos nesta seção que a álgebra TKK afim estendida definida na seção 2.2 não possui quocientes integráveis.

Definição 3.5.1. Um $\mathcal{L}(J)$-módulo $V$ chama-se módulo integrável se $V$ é $\mathcal{L}_{0}(J)$ semisimples e os geradores de $\mathcal{L}(J)$ agem localmente nilpotentemente sobre $V$.

Observamos que

$$
\begin{aligned}
{\left[e_{-}, e_{+}(m, 0)\right] } & =-h(m, 0), \\
{\left[e_{-}, e_{+}(0, n)\right] } & =-h(0, n), \\
{[h(m, 0), h(0, n)] } & =4\left[L_{x^{(m, 0)}}, L_{x^{(0, n)}}\right]
\end{aligned}
$$

Pelo Lema 2.2.1, os elementos da forma $\left[L_{x^{(m, 0)}}, L_{x^{(0, n)}}\right]=\left[L_{x^{\delta_{1}}}, L_{x^{(m, n)-\delta_{1}}}\right]$ tal que $(m, n) \in$ $S^{\perp}$ formam uma base de $\operatorname{Inder}(J)$, portanto, $e_{ \pm}, e_{ \pm}( \pm 1,0), e_{ \pm}(0, \pm 1), \mathbf{d}_{\mathbf{1}}, \mathbf{d}_{\mathbf{2}}$ geram $\mathcal{L}(J)$.

Proposição 3.5.2. Para $m_{i}, n \in \mathbb{Z}, m_{i}>0$ e $n \geq 1$, temos em $M(\lambda)$

$$
\left(\prod_{i=1}^{n} e_{+}\left(2 m_{i}, 1\right)\right) e_{-}(0,-1)^{n+1} v_{\lambda}=(-1)^{n}(n+1) ! n ! e_{-}(2 m,-1) v_{\lambda}
$$

onde $m=\sum_{i=1}^{n} m_{i}$. 
Prova: Vamos provar a igualdade para $n=1$.

$$
\begin{aligned}
e_{+}\left(2 m_{1}, 1\right) e_{-}(0,-1)^{2} v_{\lambda}= & {\left[e_{+}\left(2 m_{1}, 1\right), e_{-}(0,-1)\right] e_{-}(0,-1) v_{\lambda} } \\
& +e_{-}(0,-1)\left[e_{+}\left(2 m_{1}, 1\right), e_{-}(0,-1)\right] v_{\lambda} \\
= & h\left(2 m_{1}, 0\right) e_{-}(0,-1) v_{\lambda}+e_{-}(0,-1) h\left(2 m_{1}, 0\right) v_{\lambda} \\
= & -2 e_{-}\left(2 m_{1},-1\right) v_{\lambda} \\
= & (-1) 2 ! 1 ! e_{-}\left(2 m_{1},-1\right) v_{\lambda}
\end{aligned}
$$

Assumimos que a igualdade acima seja verdadeira para $n(n>1)$ e vamos provar para $n+1$. Seja $m=\sum_{i=1}^{n+1} m_{i}$. Temos

$$
\begin{aligned}
\prod_{i=1}^{n+1} e_{+}\left(2 m_{i}, 1\right) e_{-}(0,-1)^{n+2} v_{\lambda} & =\prod_{i=1}^{n+1} e_{+}\left(2 m_{i}, 1\right) e_{-}(0,-1)^{n+1} e_{-}(0-1) v_{\lambda} \\
& =\left[\prod_{i=1}^{n+1} e_{+}\left(2 m_{i}, 1\right) e_{-}(0,-1)^{n+1}, e_{-}(0,-1)\right] v_{\lambda} \\
& +e_{-}(0,-1) \prod_{i=1}^{n+1} e_{+}\left(2 m_{i}, 1\right) e_{-}(0,-1)^{n+1} v_{\lambda} \\
& =\left[\prod_{i=1}^{n+1} e_{+}\left(2 m_{i}, 1\right) e_{-}(0,-1)^{n+1}, e_{-}(0,-1)\right] v_{\lambda} \\
& +e_{-}(0,-1) e_{+}\left(2 m_{n+1}, 1\right) \prod_{i=1}^{n} e_{+}\left(2 m_{i}, 1\right), e_{-}(0,-1)^{n+1} v_{\lambda}
\end{aligned}
$$

Pela hipótese de indução, $\prod_{i=1}^{n} e_{+}\left(2 m_{i}, 1\right) e_{-}(0,-1)^{n+1} v_{\lambda}=(-1)^{n}(n+1) ! n ! e_{-}(2 m,-1) v_{\lambda}$, $\log \mathrm{O}$

$$
\begin{aligned}
\prod_{i=1}^{n+1} e_{+}\left(2 m_{i}, 1\right) e_{-}(0,-1)^{n+2} v_{\lambda} & =\left[\prod_{i=1}^{n+1} e_{+}\left(2 m_{i}, 1\right) e_{-}(0,-1)^{n+1}, e_{-}(0,-1)\right] v_{\lambda} \\
& +(-1)^{n}(n+1) ! n ! e_{-}(0,-1) e_{+}\left(2 m_{n+1}, 1\right) e_{-}\left(2 m-2 m_{n+1},-1\right) v_{\lambda} \\
& =\left[\prod_{i=1}^{n+1} e_{+}\left(2 m_{i}, 1\right) e_{-}(0,-1)^{n+1}, e_{-}(0,-1)\right] v_{\lambda} \\
& +(-1)^{n}(n+1) ! n ! e_{-}(0,-1)\left[e_{+}\left(2 m_{n+1}, 1\right), e_{-}\left(2 m-2 m_{m+1},-1\right)\right] v_{\lambda}
\end{aligned}
$$

Como $\left[e_{+}\left(2 m_{n+1}, 1\right), e_{-}\left(2 m-2 m_{m+1},-1\right)\right] \in U(\mathcal{L}(J))_{2 m \delta_{1}}$ e $m>0$ temos que $\left[e_{+}\left(2 m_{n+1}, 1\right), e_{-}(2 m-\right.$ $\left.\left.2 m_{m+1},-1\right)\right] v_{\lambda}=0$. Assim 


$$
\begin{aligned}
& \prod_{i=1}^{n+1} e_{+}\left(2 m_{i}, 1\right) e_{-}(0,-1)^{n+2} v_{\lambda}=\left[\prod_{i=1}^{n+1} e_{+}\left(2 m_{i}, 1\right) e_{-}(0,-1)^{n+1}, e_{-}(0,-1)\right] v_{\lambda} \\
& =\sum_{i=1}^{n+1} e_{+}\left(2 m_{1}, 1\right) \cdots h\left(2 m_{i}, 0\right) \cdots e_{+}\left(2 m_{n+1}, 1\right) e_{-}(0,-1)^{n+1} v_{\lambda} \\
& =\sum_{i=1}^{n+1}\left[e_{+}\left(2 m_{1}, 1\right) \cdots h\left(2 m_{i}, 0\right)\right] \cdots e_{+}\left(2 m_{n+1}, 1\right) e_{-}(0,-1)^{n+1} v_{\lambda} \\
& \left.+\sum_{i=1}^{n+1} h\left(2 m_{i}, 0\right) e_{+}\left(2 m_{1}, 1\right) \cdots e_{+}\left(2 m_{i-1}\right) \widehat{e\left(2 m_{i}, 1\right.}\right) \cdots e_{-}(0,-1)^{n+1} v_{\lambda} \\
& \left.=\sum_{i=1}^{n+1} \sum_{j=1}^{i-1} e_{+}\left(2 m_{1}, 1\right) \cdots\left[e_{+}\left(2 m_{j}, 1\right), h\left(2 m_{i}, 0\right)\right] \cdots e \widehat{\left(2 m_{i}, 1\right.}\right) \cdots e_{-}(0,-1)^{n+1} v_{\lambda} \\
& \left.+\sum_{i=1}^{n+1} h\left(2 m_{i}, 0\right) e_{+}\left(2 m_{1}, 1\right) \cdots e_{+} \widehat{\left(2 m_{i}\right.}, 1\right) \cdots e_{-}(0,-1)^{n+1} v_{\lambda} \\
& \left.\left.=-2 \sum_{i=1}^{n+1} \sum_{j=1}^{i-1} e_{+}\left(2 m_{1}, 1\right) \cdots e_{+}\left(2 m_{j}+2 m_{i}, 1\right) \cdots e_{+} \widehat{\left(2 m_{j}\right.}, 1\right) \cdots e_{+} \widehat{\left(2 m_{i}\right.}, 1\right) \cdots e_{-}(0,-1)^{n+1} v_{\lambda} \\
& +\sum_{i=1}^{n+1} h\left(2 m_{i}, 0\right)(-1)^{n} n !(n+1) ! e_{-}\left(2 m-2 m_{i},-1\right) v_{\lambda} \\
& =-2 \sum_{i=1}^{n+1} \sum_{j=1}^{i-1}(-1)^{n} n !(n+1) ! e_{-}(2 m,-1)-2 \sum_{i=1}^{n+1}(-1)^{n} n !(n+1) ! e_{-}(2 m,-1) v_{\lambda} \\
& =-2 \frac{n(n+1)}{2} n !(n+1) ! e_{-}(2 m,-1)-2(n+1)(-1)^{n} n !(n+1) ! e_{-}(2 m,-1) v_{\lambda} \\
& =(-1)^{n}(n+1) !(n+2) ! e_{-}(2 m,-1) v_{\lambda} .
\end{aligned}
$$

Portanto, a igualdade se verifica para todo $n \geq 1$.

Consideramos $n>0$ e $m_{i}=1$, para todo $i$, então

$$
e_{+}(2,1)^{n} e_{-}(0,-1)^{n+1} v_{\lambda}=(-1)^{n}(n+1) ! n ! e_{-}(2 n,-1) v_{\lambda}
$$

Multiplicando a equação acima por $e_{+}(-2(n+K), 1)$, para $K>0$, temos

$$
\begin{aligned}
e_{+}(-2(n+K), 1) e_{+}(2,1)^{n} e_{-}(2 n,-1) v_{\lambda} & =(-1)^{n}(n+1) ! n ! e_{+}(-2(n+K), 1) e_{-}(2 n,-1) v_{\lambda} \\
& =(-1)^{n}(n+1) ! n ! h(-2 K, 0) v_{\lambda}
\end{aligned}
$$


Seja $N$ o submódulo de $M(\lambda)$ gerado por $e_{-}(0,-1)^{n} v_{\lambda}$, para algum $n>0$ fixo. Pela equação acima temos $h(-2 K, 0) v_{\lambda} \in N$ e, para $0<s<2 K$, temos

$$
e_{+}(s, 0) h(-2 K, 0) v_{\lambda}=-2 e_{+}(-2 K+s, 0) v_{\lambda} \in N
$$

Como $K$ é um inteiro positivo arbitrário, concluímos que $e_{+}(-r, 0) v_{\lambda} \in N$, para todo $r>0$.

Já vimos que $e_{-}(2 n,-1) v_{\lambda} \in N$. Logo

$$
\begin{aligned}
h(0,-1) v_{\lambda} & =\left[e_{+}(-2 n, 0), e_{-}(2 n,-1)\right] v_{\lambda} \\
& =e_{+}(-2 n, 0) e_{-}(2 n,-1) v_{\lambda}-e_{-}(2 n,-1) e_{+}(-2 n, 0) v_{\lambda} \in N
\end{aligned}
$$

Observamos que

$$
h(0,1) h(0,-1) v_{\lambda}=(h, h) \mathbf{c}_{\mathbf{2}} v_{\lambda} \in N
$$

e

$$
h(2 K, 0) h(-2 K, 0) v_{\lambda}=2 K(h, h) \mathbf{c}_{1} v_{\lambda} \in N,
$$

portanto, se $\lambda\left(\mathbf{c}_{\mathbf{1}}\right) \neq 0$ ou $\lambda\left(\mathbf{c}_{\mathbf{2}}\right) \neq 0$, então $e_{-}(0,-1)^{n+1} v_{\lambda}$ gera, como submódulo, todo o $M(\lambda)$.

Por outro lado, se $\lambda\left(\mathbf{c}_{\mathbf{1}}\right)=0, \lambda\left(\mathbf{c}_{\mathbf{2}}\right)=0$ e $\lambda(h) \neq 0$, pelo fato que

$$
\begin{aligned}
e_{+}(-2 n, 1) e_{+}(2,1)^{n} e_{-}(0,-1)^{n+1} v_{\lambda} & =(-1)^{n}(n+1) ! n ! e_{+}(-2 n, 1) e_{-}(2 n,-1) v_{\lambda} \\
& =(-1)^{n}(n+1) ! n ! h(0,0) v_{\lambda} \\
& =(-1)^{n}(n+1) ! n ! \lambda(h) \in N
\end{aligned}
$$

novamente $e_{-}(0,-1)^{n+1} v_{\lambda}$ irá gerar todo o $M(\lambda)$.

Seja $I$ um quociente integrável de $M(\lambda)$, então

$$
\phi: M(\lambda) \longrightarrow M(\lambda) / N=I
$$

para algum submódulo $N$ de $M(\lambda)$.

A imagem de $\phi\left(e_{-}(0,-1)^{n+1} v_{\lambda}\right)$ é 0 , para algum $n$, pela definição de módulo integrável. Assim, se $\lambda \neq 0$ então $e_{-}(0,-1)^{n+1} v_{\lambda}$ gera todo módulo o $M(\lambda)$ e, portanto, $M(\lambda) / N=$ $I=\{0\}$ o que significa que $M(\lambda)$ não possui quocientes integráveis. 


\section{Capítulo 4}

\section{Módulo de Verma Imaginário}

O principal resultado que provamos nesse capítulo é um critério de irredutibilidade para o módulo de Verma imaginário sobre a álgebra TKK afim estendida da seção 2.2. A irredutibilidade é determinado pelo módulo de Verma sobre uma subálgebra tipo Heisenberg.

\subsection{Subálgebra tipo Heisenberg}

Seja

$$
G=\left(\oplus_{\sigma \in R^{0} \backslash\{0\}} \mathcal{G}_{0}^{\sigma}(J)\right) \oplus \mathcal{C} \oplus \mathcal{D}
$$

auma subálgebra tipo Heisenberg de $\mathcal{L}(J)$. Lembramos que

$$
\mathcal{G}_{0}^{\sigma}(J)= \begin{cases}\mathbb{C} h \otimes x^{\sigma} & , \text { se } \sigma \in S, \\ \mathbb{C}\left[L_{x^{\delta_{1}}}, L_{x^{\sigma-\delta_{1}}}\right] & , \text { se } \sigma \in S^{\perp}\end{cases}
$$

e o produto em $G$ será:

$$
\begin{aligned}
{[\mathcal{C}, G] } & =[\mathcal{D}, \mathcal{D}]=\{0\} \\
{\left[\mathbf{d}_{\mathbf{i}}, y\right] } & =\delta_{i} \cdot \sigma y, \text { para } y \in \mathcal{G}_{0}^{\sigma}(J)
\end{aligned}
$$

e

$$
[h(\sigma), h(\tau)]= \begin{cases}2 \Omega(\tau) h(\sigma+\tau) & , \text { se } \sigma \in S^{\perp}, \tau \in S, \\ -4 \delta_{\sigma+\tau, 0}\left(\sigma \cdot \delta_{1} \mathbf{c}_{\mathbf{1}}+\sigma \cdot \delta_{2} \mathbf{c}_{\mathbf{2}}\right) & , \text { se } \sigma, \tau \in S^{\perp}, \\ 4 \delta_{\sigma+\tau, 0}\left(\sigma \cdot \delta_{1} \mathbf{c}_{\mathbf{1}}+\sigma \cdot \delta_{2} \mathbf{c}_{\mathbf{2}}\right) & , \text { se } \sigma, \tau \in S, \sigma+\tau \in S, \\ 2 \Omega(\tau) h(\sigma+\tau) & , \text { se } \sigma, \tau \in S, \sigma+\tau \in S^{\perp}\end{cases}
$$


onde $\Omega(\tau)=\frac{1}{2}\left((-1)^{\tau \cdot \delta_{1}}-(-1)^{\tau \cdot \delta_{2}}\right)$.

Observação 4.1.1. Dado $h(\sigma) \in G$ podemos obter $h(\tau)$ tal que $[h(\sigma), h(\tau)]=k h(\sigma+\tau) \neq$ 0 , para algum $k \in \mathbb{C}^{*}$. De fato, se $\sigma \in S$ (resp. $\sigma \in S^{\perp}$ ) escolhemos $\tau \in S^{\perp}$ (resp. $\tau \in S$ ).

Denotamos por

$$
\begin{aligned}
& P_{+}^{1}=\left\{\sigma \in R_{+}^{0} \mid \sigma=(m, n), n=0\right\} \\
& P_{+}^{2}=\left\{\sigma \in R_{+}^{0} \mid \sigma=(m, n), n \neq 0\right\}
\end{aligned}
$$

e por

$$
\begin{aligned}
G_{ \pm 1} & =\bigoplus_{\sigma \in P_{+}^{1}} \mathcal{G}_{0}^{ \pm \sigma}(J) \\
G_{ \pm 2} & =\bigoplus_{\sigma \in P_{+}^{2}} \mathcal{G}_{0}^{ \pm \sigma}(J) \\
G_{ \pm} & =G_{ \pm 1}+G_{ \pm 2}
\end{aligned}
$$

Assim, podemos decompor $G$ da seguinte forma

$$
G=G_{-} \oplus G_{0} \oplus G_{+}
$$

onde $G_{0}=\mathcal{C} \oplus \mathcal{D}$

\subsubsection{Módulo de Verma sobre a subálgebra tipo Heisenberg}

Seja $\tau \in\left(G_{0}\right)^{*}$. Consideramos o módulo de Verma para $G$ :

$$
\tilde{M}\{\tau\}=U(G) \otimes_{U\left(G_{0} \oplus G_{+}\right)} \mathbb{C} v_{+}
$$

induzido de um $G_{0} \oplus G_{+}$-módulo de dimensão um $\mathbb{C} v_{+}$, com a ação dada por $(h+c) v_{+}=$ $\tau(c) v_{+}$, para todo $h \in G_{+}$e $c \in G_{0}$.

A álgebra envolvente universal $U(G)$ é $\mathbb{Z}^{2}$-graduada e dizemos que um elemento em $U(G)_{m \delta_{1}+n \delta_{2}}$ possui grau $(n, m)$. Claramente $\tilde{M}\{\tau\}$ é $\mathbb{Z}^{2}$-graduada e livre sobre $U\left(G_{-}\right)$.

Proposição 4.1.2. Seja $\tau \in G_{0}^{*}$ e $\mu=(m, n) \in \mathbb{Z}^{2}$. Então

(i) $\tilde{M}\{\tau\}$ possui um único submódulo próprio maximal $\mathbb{Z}^{2}$-graduado $\tilde{N}$; 
(ii) A menos de isomorfismo $L=\tilde{M}\{\tau\} / \tilde{N}$ é o único módulo de peso máximo irredutível de $G$ com peso máximo $\tau$;

(iii) Se $V$ é um G-módulo $\mathbb{Z}^{2}$-graduado gerado por um elemento $v$ tal que $G_{+} v=\{0\}$ e $c v=\tau(c) v$, para todo $c \in \mathcal{C}$, então $V$ é uma imagem homomórfica de $\tilde{M}\{\tau\}$.

Prova: (i) Segue do fato que a soma de submódulos próprios de $\tilde{M}\{\tau\}$ é um submódulo próprio. (ii) segue de (i) e (iii) segue diretamente da construção do módulo $\tilde{M}\{\tau\}$.

\subsection{Base e ordem}

Consideramos o sistema de raízes positivo normal $P_{1}=(\dot{\alpha}+S) \cup R_{+}^{0}$.

Denotamos por

$$
\mathcal{L}_{ \pm 1}:=G_{ \pm}
$$

e por

$$
\mathcal{L}_{ \pm 2}=\sum_{\alpha \in P_{1} \cap R^{\times}} \mathcal{L}_{ \pm \alpha}(J) .
$$

Seja

$$
\mathcal{L}_{ \pm}=\mathcal{L}_{ \pm 1}+\mathcal{L}_{ \pm 2}
$$

Então

$$
\mathcal{L}(J)=\mathcal{L}_{+} \oplus \mathcal{L}_{0}(J) \oplus \mathcal{L}_{-},
$$

onde $\mathcal{L}_{0}(J)=\mathcal{G}_{0}(J) \cap \mathcal{G}^{0}(J) \oplus \mathcal{C} \oplus \mathcal{D}$.

O conjunto $\left\{\mathbf{c}_{\mathbf{1}}, \mathbf{c}_{\mathbf{2}}, \mathbf{d}_{\mathbf{1}}, \mathbf{d}_{\mathbf{2}}, h(m, n) \mid(m, n) \in \mathbb{Z}^{2}, n>0\right\} \cup\{h(m, 0) \mid m>0\}$ forma uma base para $G_{+} \oplus G_{0}$ e $\left\{h(m, n) \mid(m, n) \in \mathbb{Z}^{2}, n<0\right\} \cup\{h(m, 0) \mid m<0\}$ forma uma base de $G_{-}$.

Seja $\Theta\left(\mathcal{L}_{-}\right)=\left\{e_{-}(m, n) \mid(m, n) \in S\right\} \cup\left\{h(m, n) \mid(m, n) \in \mathbb{Z}^{2}, n<0\right\} \cup\{h(m, 0) \mid$ $m<0\}$ uma base de $\mathcal{L}_{-}$.

Definimos uma ordem total em $\Theta\left(\mathcal{L}_{-}\right)$declarando que $x_{1}\left(m_{1}, n_{1}\right)<x_{2}\left(m_{2}, n_{2}\right)$ se ou $\beta_{1}<\beta_{2}$ ou $\beta_{1}=\beta_{2}$ e $n_{1}<n_{2}$ ou $\beta_{1}=\beta_{2}, n_{1}=n_{2}$ e $m_{1}<m_{2}$, onde $\beta_{1}, \beta_{2}$ são as raízes associadas a $x_{1}, x_{2}$, respectivamente. 
Uma base de $U\left(\mathcal{L}_{-}\right)$consiste de monômios da forma

$$
x_{i_{1}}\left(m_{1}, n_{1}\right) x_{i_{2}}\left(m_{2}, n_{2}\right) \cdots x_{i_{r}}\left(m_{r}, n_{r}\right),
$$

$\operatorname{com} x_{i_{1}}\left(m_{1}, n_{1}\right) \leq x_{i_{2}}\left(m_{2}, n_{2}\right) \leq \cdots \leq x_{i_{r}}\left(m_{r}, n_{r}\right)$.

Denotamos por

$$
x_{i, m, n, p}:=x_{i_{1}}\left(m_{1}, n_{1}\right)^{p_{1}} x_{i_{2}}\left(m_{2}, n_{2}\right)^{p_{2}} \cdots x_{i_{r}}\left(m_{r}, n_{r}\right)^{p_{r}}
$$

os elementos em $U\left(\mathcal{L}_{-}\right) \operatorname{com} p_{j} \geq 1$ e $x_{i_{1}}\left(m_{1}, n_{1}\right)<\cdots<x_{i_{r}}\left(m_{r}, n_{r}\right)$. Então

$$
\Theta\left(U\left(\mathcal{L}_{-}\right)\right)=\left\{x_{i, m, n, p}\right\} \cup\{1\}
$$

forma uma base de $U\left(\mathcal{L}_{-}\right)$.

Sejam

$$
\Theta_{1}=\left\{x_{i, m, n, p} \in \Theta\left(U\left(\mathcal{L}_{-}\right)\right) \mid \beta_{i_{j}}=0, \text { para todo } j=i_{1}, \cdots, i_{r}\right\}
$$

e

$$
\Theta_{2}=\left\{x_{i, m, n, p} \in \Theta\left(U\left(\mathcal{L}_{-}\right)\right) \mid \beta_{i_{j}}=-\dot{\alpha}, \text { para todo } j=i_{1}, \cdots, i_{r}\right\}
$$

Então $\Theta\left(U\left(\mathcal{L}_{-}\right)\right)=\Theta_{2} \Theta_{1}=\left\{\theta_{2} \theta_{1} \mid \theta_{i} \in \Theta_{i}, i=1,2\right\}$ e $\Theta_{1}$ (respectivamente $\left.\Theta_{2}\right)$ forma uma base de $U\left(\mathcal{L}_{-1}\right)$ (respectivamente $U\left(\mathcal{L}_{-2}\right)$ ). Para simplicar denotamos por $x_{m, n, p}^{0}=$ $x_{0}\left(m_{1}, n_{1}\right)^{p_{1}} \cdots x_{0}\left(m_{r}, n_{r}\right)^{p_{r}}$ os elementos $U\left(\mathcal{L}_{-1}\right)$ e por $x_{m, n, p}^{-\dot{\alpha}}=x_{-\dot{\alpha}}\left(m_{1}, n_{1}\right)^{p_{1}} \cdots x_{-\dot{\alpha}}\left(m_{r}, n_{r}\right)^{p_{r}}$ os elementos $U\left(\mathcal{L}_{-2}\right)$. Definimos uma ordem total em $\Theta_{1}$ (respectivamente $\left.\Theta_{2}\right)$ : como segue onde $\beta=0$ (respectivamente $\beta=-\dot{\alpha}$ )

$$
x_{m, n, p}^{\beta}=x_{\beta}\left(m_{1}, n_{1}\right)^{p_{1}} \cdots x_{\beta}\left(m_{r}, n_{r}\right)^{p_{r}}<x_{\beta}\left(k_{1}, l_{1}\right)^{q_{1}} \cdots x_{\beta}\left(k_{s}, l_{s}\right)^{q_{s}}=x_{k, l, q}^{\beta}
$$

se, e somente se, existe um $\gamma \geq 0$ tal que

$$
x_{\beta}\left(m_{\gamma}, n_{\gamma}\right)^{p_{\gamma}} \cdots x_{\beta}\left(m_{r}, n_{r}\right)^{p_{r}}=x_{\beta}\left(k_{\gamma}, l_{\gamma}\right)^{q_{\gamma}} \cdots x_{\beta}\left(k_{s}, l_{s}\right)^{q_{s}}
$$

e

$$
\begin{aligned}
& \text { ou } x_{\beta}\left(m_{\gamma-1}, n_{\gamma-1}\right)^{p_{\gamma-1}}<x_{\beta}\left(k_{\gamma-1}, l_{\gamma-1}\right)^{q_{\gamma-1}} \\
& \text { ou } x_{\beta}\left(m_{\gamma-1}, n_{\gamma-1}\right)^{p_{\gamma-1}}=x_{\beta}\left(k_{\gamma-1}, l_{\gamma-1}\right)^{q_{\gamma-1}} \text { e } p_{\gamma-1}>q_{\gamma-1} .
\end{aligned}
$$


Caso $r>s$ e $x_{\beta}\left(m_{r-s+1}, n_{r-s+1}\right)^{p_{r-s+1}} \cdots x_{\beta}\left(m_{r}, n_{r}\right)^{p_{r}}=x_{\beta}\left(k_{1}, l_{1}\right)^{q_{1}} \cdots x_{\beta}\left(k_{s}, l_{s}\right)^{q_{s}}$ convencionamos que $x_{m, n, p}^{\beta}<x_{k, l, q}^{\beta}$. Isso significa que $x_{\beta}\left(m_{r-s}, n_{r-s}\right)<1$.

Para $\theta_{2} \theta_{1}, \theta_{2}^{\prime} \theta_{1}^{\prime} \in \Theta\left(U\left(\mathcal{L}_{-}\right)\right)=\Theta_{2} \Theta_{1}$ dizemos que

$$
\theta_{2} \theta_{1}<\theta_{2}^{\prime} \theta_{1}^{\prime}
$$

se ou $\theta_{2}<\theta_{2}^{\prime}$ ou $\theta_{2}=\theta_{2}^{\prime}$ e $\theta_{1}<\theta_{1}^{\prime}$. Isso define uma ordem total em $B\left(U\left(\mathcal{L}_{-}\right)\right)$que estende a ordem definida sobre $\Theta_{1}$ e $\Theta_{2}$.

\subsection{Módulo de Verma imaginário}

Seja $\lambda \in \mathcal{L}_{0}(J)^{*}$. Denotamos por

$$
M\{\lambda\}:=M_{B_{P_{1}}}(\lambda)
$$

o módulo tipo Verma associado a $B_{P_{1}}$ e $\lambda$ chamado módulo de Verma imaginário.

Para $\lambda \in \mathcal{L}_{0}(J)^{*}$ denotamos por $\tilde{\lambda}$ a restrição de $\lambda$ a $\mathcal{C}^{*}$. Então $\tilde{M}:=\tilde{M}\{\tilde{\lambda}\}$ é um $G$-submódulo de $M\{\lambda\}$.

Para $N$ um submódulo de $M\{\lambda\}$ denotamos por $\tilde{N}:=N \cap \tilde{M}$.

Proposição 4.3.1. Seja $N$ um submódulo não-nulo de $M\{\lambda\}$. Então $\tilde{N}$ é não-nulo.

Prova: Seja $0 \neq u v_{+} \in N$ com $u \in U\left(\mathcal{L}_{-}\right)$. Por ([14], Seção 2.1, Proposição 1) podemos assumir que $u$ é um elemento homogêneo e pelo Teorema de PBW podemos escrevê-lo da seguinte forma:

$$
u=\sum_{i \in I} u_{i} u_{i}^{\prime}
$$

onde $u_{i}^{\prime} \in U\left(G_{-}\right)$e

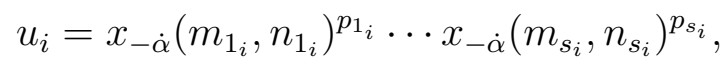

onde $x_{-\dot{\alpha}}\left(m_{1_{i}}, n_{1_{i}}\right)<\cdots<x_{-\dot{\alpha}}\left(m_{s_{i}}, n_{s_{i}}\right)$ e os $u_{i}$ são linearmente independentes.

Vamos provar por indução sobre $h t(u):=\sum_{j=p_{1_{i}}}^{p_{s_{i}}} j$, que existe um elemento $0 \neq z \in$ $U(\mathcal{L}(J))$ tal que $0 \neq z u v_{+} \in \tilde{M}$. Observamos que $h t u$ independe de $i$ pois $u$ é um elemento homogêneo. 
Supondo que $h t(u)=1$, então

$$
u=\sum_{i \in I} x_{-\dot{\alpha}}\left(m_{i}, n_{i}\right) u_{i}^{\prime}
$$

com $x_{-\dot{\alpha}}\left(m_{i}, n_{i}\right)$ linearmente independentes. Seja $z=x_{\dot{\alpha}}(-M,-N) \operatorname{com}(-M,-N) \in$ $S$ e $M, N>>$. Então, $0 \neq\left[z, x_{-\dot{\alpha}}\left(m_{i}, n_{i}\right)\right]=\left[x_{\dot{\alpha}}(-M,-N), x_{-\dot{\alpha}}\left(m_{i}, n_{i}\right)\right] \in G_{-}$são linearmente independentes e

$$
0 \neq z u v_{+}=\sum_{i \in I}\left[x_{\dot{\alpha}}(-M,-N), x_{-\dot{\alpha}}\left(m_{i}, n_{i}\right)\right] u_{i}^{\prime} v_{+} \in \tilde{M}
$$

Agora suponhamos que $h t(u)>1$ e assumimos que o resultado se verifica para todo elemento homogêneo em $N$ com altura menor do que $h t(u)$. O elemento $u$ será da seguinte forma

$$
u=\sum_{i \in I} u_{i} u_{i}^{\prime}
$$

Denotamos por $u_{a}$ o elemento maximal entre os $u_{i} \operatorname{com} i \in I$. Consideramos o elemento $y=x_{\dot{\alpha}}(-M,-N)$ tal que $(-M,-N) \in S, M, N>>0$, agindo sobre $u_{a} u_{a}^{\prime} v_{+}$: $y u_{a} u_{a}^{\prime} v_{+}=\sum_{j=1_{a}}^{s_{a}} x_{-\dot{\alpha}}\left(m_{1_{a}}, n_{1_{a}}\right)^{p_{1_{a}}} \cdots\left[x_{\dot{\alpha}}(-M,-N), x_{-\dot{\alpha}}\left(m_{j}, n_{j}\right)^{p_{j}}\right] \cdots x_{-\dot{\alpha}}\left(m_{s_{a}}, n_{s_{a}}\right)^{p_{s_{a}}} u_{a}^{\prime} v_{+}$ e $\left[x_{\dot{\alpha}}(-M,-N), x_{-\dot{\alpha}}\left(m_{j}, n_{j}\right)^{p_{j}}\right]=\sum_{l=0}^{p_{j}-1} x_{-\dot{\alpha}}\left(m_{j}, n_{j}\right)^{l}\left[x_{\dot{\alpha}}(-M,-N), x_{-\dot{\alpha}}\left(m_{j}, n_{j}\right)\right] x_{-\dot{\alpha}}\left(m_{j}, n_{j}\right)^{p_{j}-1-l}$. 
Assim,

$$
\begin{aligned}
& y u_{a} u_{a}^{\prime} v_{+}=\sum_{j=1_{a}}^{s_{a}} \sum_{l=0}^{p_{j}-1} x_{-\dot{\alpha}}\left(m_{1_{a}}, n_{1_{a}}\right)^{p_{1_{a}}} \cdots x_{-\dot{\alpha}}\left(m_{j}, n_{j}\right)^{l}\left[\left[x_{\dot{\alpha}}(-M,-N), x_{-\dot{\alpha}}\left(m_{j}, n_{j}\right)\right],\right. \\
& \left.x_{-\dot{\alpha}}\left(m_{j}, n_{j}\right)^{p_{j}-1-l} \cdots x_{-\dot{\alpha}}\left(m_{s_{a}}, n_{s_{a}}\right)^{p_{s_{a}}}\right] u_{a}^{\prime} v_{+} \\
& +\sum_{j=1_{a}}^{s_{a}} \sum_{l=0}^{p_{j}-1} x_{-\dot{\alpha}}\left(m_{1_{a}}, n_{1_{a}}\right)^{p_{1_{a}}} \cdots x_{-\dot{\alpha}}\left(m_{j}, n_{j}\right)^{l} x_{-\dot{\alpha}}\left(m_{j}, n_{j}\right)^{p_{j}-1-l} \cdots \\
& x_{-\dot{\alpha}}\left(m_{s_{a}}, n_{s_{a}}\right)^{p_{s_{a}}}\left[x_{\dot{\alpha}}(-M,-N), x_{-\dot{\alpha}}\left(m_{j}, n_{j}\right)\right] u_{a}^{\prime} v_{+} \\
& =\sum_{j=1_{a}}^{s_{a}} \sum_{l=0}^{p_{j}-1} x_{-\dot{\alpha}}\left(m_{1_{a}}, n_{1_{a}}\right)^{p_{1_{a}}} \cdots x_{-\dot{\alpha}}\left(m_{j}, n_{j}\right)^{l}\left[\left[x_{\dot{\alpha}}(-M,-N), x_{-\dot{\alpha}}\left(m_{j}, n_{j}\right)\right],\right. \\
& \left.x_{-\dot{\alpha}}\left(m_{j}, n_{j}\right)^{p_{j}-1-l} \cdots x_{-\dot{\alpha}}\left(m_{s_{a}}, n_{s_{a}}\right)^{p_{s_{a}}}\right] u_{a}^{\prime} v_{+}
\end{aligned}
$$

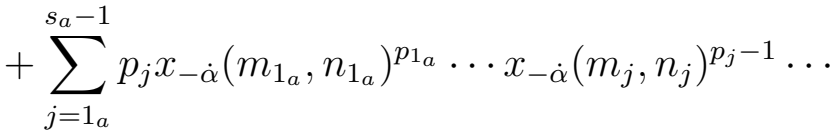

$$
\begin{aligned}
& x_{-\dot{\alpha}}\left(m_{s_{a}}, n_{s_{a}}\right)^{p_{s_{a}}}\left[x_{\dot{\alpha}}(-M,-N), x_{-\dot{\alpha}}\left(m_{j}, n_{j}\right)\right] u_{a}^{\prime} v_{+} \\
& +p_{s_{a}} x_{-\dot{\alpha}}\left(m_{1_{a}}, n_{1_{a}}\right)^{p_{1_{a}}} \cdots x_{-\dot{\alpha}}\left(m_{s_{a}}, n_{s_{a}}\right)^{p_{s_{a}}-1}\left[x_{\dot{\alpha}}(-M,-N), x_{-\dot{\alpha}}\left(m_{s_{a}}, n_{s_{a}}\right)\right] u_{a}^{\prime} v_{+}
\end{aligned}
$$

Agora denotamos por

$$
\begin{aligned}
& T_{1}=\sum_{j=1_{a}}^{s_{a}} \sum_{l=0}^{p_{j}-1} x_{-\dot{\alpha}}\left(m_{1_{a}}, n_{1_{a}}\right)^{p_{1 a}} \cdots x_{-\dot{\alpha}}\left(m_{j}, n_{j}\right)^{l}\left[\left[x_{\dot{\alpha}}(-M,-N), x_{-\dot{\alpha}}\left(m_{j}, n_{j}\right)\right],\right. \\
& \left.x_{-\dot{\alpha}}\left(m_{j}, n_{j}\right)^{p_{j}-1-l} \cdots x_{-\dot{\alpha}}\left(m_{s_{a}}, n_{s_{a}}\right)^{p_{s_{a}}}\right] u_{a}^{\prime} v_{+} \\
& T_{2}=\sum_{j=1_{a}}^{s_{a}-1} p_{j} x_{-\dot{\alpha}}\left(m_{1_{a}}, n_{1_{a}}\right)^{p_{1_{a}}} \cdots x_{-\dot{\alpha}}\left(m_{j}, n_{j}\right)^{p_{j}-1} \ldots \\
& x_{-\dot{\alpha}}\left(m_{s_{a}}, n_{s_{a}}\right)^{p_{s_{a}}}\left[x_{\dot{\alpha}}(-M,-N), x_{-\dot{\alpha}}\left(m_{j}, n_{j}\right)\right] u_{a}^{\prime} v_{+} \\
& T_{3}=p_{s_{a}} x_{-\dot{\alpha}}\left(m_{1_{a}}, n_{1_{a}}\right)^{p_{1_{a}}} \cdots x_{-\dot{\alpha}}\left(m_{s_{a}}, n_{s_{a}}\right)^{p_{s_{a}}-1}\left[x_{\dot{\alpha}}(-M,-N), x_{-\dot{\alpha}}\left(m_{s_{a}}, n_{s_{a}}\right)\right] u_{a}^{\prime} v_{+} .
\end{aligned}
$$

Denotamos também por

$$
\begin{gathered}
T_{1_{j l}}^{-\dot{\alpha}}=x_{-\dot{\alpha}}\left(m_{1_{a}}, n_{1_{a}}\right)^{p_{1 a}} \cdots x_{-\dot{\alpha}}\left(m_{j}, n_{j}\right)^{l}\left[\left[x_{\dot{\alpha}}(-M,-N), x_{-\dot{\alpha}}\left(m_{j}, n_{j}\right)\right]\right. \\
\left.x_{-\dot{\alpha}}\left(m_{j}, n_{j}\right)^{p_{j}-1-l} \cdots x_{-\dot{\alpha}}\left(m_{s_{a}}, n_{s_{a}}\right)^{p_{s_{a}}}\right]
\end{gathered}
$$


para $j=1_{a}, \cdots, s_{a}$ e $l=0, \cdots, p_{j}-1$ e

$$
T_{3}^{-\dot{\alpha}}=x_{-\dot{\alpha}}\left(m_{1_{a}}, n_{1_{a}}\right)^{p_{1_{a}}} \cdots x_{-\dot{\alpha}}\left(m_{s_{a}}, n_{s_{a}}\right)^{p_{s_{a}}-1} .
$$

Observamos que $T_{3} \neq 0$ pois $\left[x_{\dot{\alpha}}(-M,-N), x_{-\dot{\alpha}}\left(m_{j}, n_{j}\right)\right]=k h\left(-M+m_{s_{a}},-N+n_{s_{a}}\right) \neq$ 0 e $M, N>>0$ com $k=1$ ou -1 .

O termo $T_{1}$ não contribui para cancelar $T_{3}$, pois

$$
\left[\left[x_{\dot{\alpha}}(-M,-N), x_{-\dot{\alpha}}\left(m_{j}, n_{j}\right)\right], x_{-\dot{\alpha}}\left(m_{j}, n_{j}\right)^{p_{j}-1-l} \cdots x_{-\dot{\alpha}}\left(m_{s_{a}}, n_{s_{a}}\right)^{p_{s_{a}}}\right] \in U\left(\mathcal{L}_{-2}\right) \mathcal{L}_{-2}
$$

e pela escolha de $M, N>>0$ temos

$$
\operatorname{deg}_{2} T_{1_{j l}}^{-\dot{\alpha}} \neq \operatorname{deg}_{2} T_{3}^{-\dot{\alpha}}
$$

para todo $j$ e $l$.

O termo $T_{2}$ não contribui para cancelar $T_{3}$, pois as potências de $x_{-\dot{\alpha}}\left(m_{s_{a}}, n_{s_{a}}\right)$ nos monômios envolvidos em $T_{2}$ e em $T_{3}$ não coincidem.

Consideramos a ação de $y$ sobre um termo arbitrário $u_{i} u_{i}^{\prime} v_{+}$de $u v_{+}$tal que $i \neq a$. Então

$$
y u_{i} u_{i}^{\prime} v_{+}=S_{1}+S_{2}
$$

onde

$$
\begin{aligned}
& S_{1}=\sum_{j=1_{i}}^{s_{i}} \sum_{l=0}^{p_{j}-1} x_{-\dot{\alpha}}\left(m_{1_{i}}, n_{1_{i}}\right)^{p_{1_{i}}} \cdots x_{-\dot{\alpha}}\left(m_{j}, n_{j}\right)^{l}\left[\left[x_{\dot{\alpha}}(-M,-N), x_{-\dot{\alpha}}\left(m_{j}, n_{j}\right)\right],\right. \\
& \left.x_{-\dot{\alpha}}\left(m_{j}, n_{j}\right)^{p_{j}-1-l} \cdots x_{-\dot{\alpha}}\left(m_{s_{i}}, n_{s_{i}}\right)^{p_{s_{i}}}\right] u_{i}^{\prime} v_{+}
\end{aligned}
$$

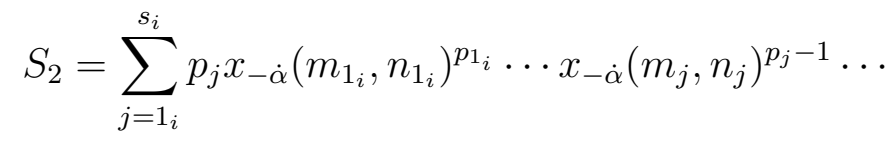

$$
\begin{aligned}
& x_{-\dot{\alpha}}\left(m_{s_{i}}, n_{s_{i}}\right)^{p_{s_{i}}}\left[x_{\dot{\alpha}}(-M,-N), x_{-\dot{\alpha}}\left(m_{j}, n_{j}\right)\right] u_{i}^{\prime} v_{+}
\end{aligned}
$$

O termo $S_{1}$ não contribui para cancelar $T_{3}$ pelo mesmo argumento usado para $T_{1}$.

Supondo que $S_{2}$ contribua para cancelar $T_{3}$, temos

$$
\begin{aligned}
x_{-\dot{\alpha}}\left(m_{1_{i}}, n_{1_{i}}\right)^{p_{1_{i}} \cdots} & x_{-\dot{\alpha}}\left(m_{j}, n_{j}\right)^{p_{j}-1} \cdots x_{-\dot{\alpha}}\left(m_{s_{i}}, n_{s_{i}}\right)^{p_{s_{i}}} \\
& =x_{-\dot{\alpha}}\left(m_{1_{a}}, n_{1_{a}}\right)^{p_{1_{a}}} \cdots x_{-\dot{\alpha}}\left(m_{s_{a}}, n_{s_{a}}\right)^{p_{s_{a}}-1}
\end{aligned}
$$


e

$$
\left[x_{\dot{\alpha}}(-M,-N), x_{-\dot{\alpha}}\left(m_{j}, n_{j}\right)\right] u_{i}^{\prime}=\left[x_{\dot{\alpha}}(-M,-N), x_{-\dot{\alpha}}\left(m_{s_{a}}, n_{s_{a}}\right)\right] u_{a}^{\prime},
$$

para algum $j=1_{i}, \cdots, s_{i}$. Como $M, N>>0$, a ordem é preservada nos monômios que aparecem na última igualdade e como a igualdade é termo a termo temos

$$
\left[x_{\dot{\alpha}}(-M,-N), x_{-\dot{\alpha}}\left(m_{j}, n_{j}\right)\right]=\left[x_{\dot{\alpha}}(-M,-N), x_{-\dot{\alpha}}\left(m_{s_{a}}, n_{s_{a}}\right)\right]
$$

e $u_{i}^{\prime}=u_{a}^{\prime}$.

Por (4.2), $u_{i}^{\prime}=u_{a}$, e pela maximalidade de $u_{a}$, temos $x_{-\dot{\alpha}}\left(m_{j}, n_{j}\right)<x_{-\dot{\alpha}}\left(m_{s_{a}}, n_{s_{a}}\right)$, o que implica $\left[x_{\dot{\alpha}}(-M,-N), x_{-\dot{\alpha}}\left(m_{j}, n_{j}\right)\right] \neq\left[x_{\dot{\alpha}}(-M,-N), x_{-\dot{\alpha}}\left(m_{s_{a}}, n_{s_{a}}\right)\right]$, contradizendo (4.4). Logo $S_{2}$ não pode contribuir para cancelar $T_{3}$ e, portanto, $y u v_{+} \neq 0$.

Como $h t(y u)=h t(u)-1$, podemos aplicar a hipótese de indução sobre $y u v_{+} \cdot$ Logo existe um termo $w \in U(\mathcal{L}(J))$ tal que $0 \neq w y u v_{+} \in \tilde{M}$ e com isso concluímos a prova da proposição.

Lema 4.3.2. Seja $N$ um submódulo não-nulo de $M\{\lambda\}$. Seja $u v_{+} \in N$ tal que

$$
u=\sum_{i \in I} h\left(M_{i}, N_{i}\right) u_{i}
$$

com $u_{i} \in U\left(G_{-}\right), h\left(M_{i}, N_{i}\right)$ linearmente independentes, $N_{i}<0, \max _{i \in I}\left\{\left[M_{i} / 2\right]\right\}<$ $\min _{i \in I}\left\{\operatorname{deg}_{1} u_{i}\right\}$ e $\max _{i \in I}\left\{\left[N_{i} / 2\right]\right\}<\min _{i \in I}\left\{\operatorname{deg}_{2} u_{i}\right\}$. Se $m \lambda\left(\mathbf{c}_{\mathbf{1}}\right)+n \lambda\left(\mathbf{c}_{\mathbf{2}}\right) \neq 0$, para todo $(m, n) \in \mathbb{Z}^{2},(m, n) \neq(0,0)$, então $u_{i} v_{+} \in N$ para todo $i \in I$.

Prova: Provaremos por indução sobre $|I|$. Supondo que $|I|=1$. Então,

$$
u=h(M, N) u^{\prime} v_{+}
$$

Consideramos o elemento $h(-M,-N)$ agindo sobre $u v_{+}$,

$$
h(-M,-N) u v_{+}=[h(-M,-N), h(M, N)] u^{\prime} v_{+}=k\left(M \lambda\left(\mathbf{c}_{\mathbf{1}}\right)+N \lambda\left(\mathbf{c}_{\mathbf{2}}\right)\right) u^{\prime} v_{+},
$$

para algum $k \in \mathbb{Z}^{*}$. Como $M \lambda\left(\mathbf{c}_{\mathbf{1}}\right)+N \lambda\left(\mathbf{c}_{\mathbf{2}}\right) \neq 0$, temos $u^{\prime} v_{+} \in N$.

Agora suponhamos que $|I|>1$. Então

$$
u=\sum_{i \in I} h\left(M_{i}, N_{i}\right) u_{i}
$$


Caso 1: Suponha que todos os $\left(M_{i}, N_{i}\right)$ pertençam a somente um do subconjuntos $S_{0}, S_{1}, S_{2}$ ou $S^{\perp}$. Neste caso, fixamos um $i_{0} \in I$ e considere $h\left(-M_{i_{0}},-N_{i_{0}}\right)$. Então

$$
\left[h\left(-M_{i_{0}},-N_{i_{0}}\right), h\left(M_{i}, N_{i}\right)\right]=0
$$

para $i \neq i_{0}$, e

$$
0 \neq h\left(-M_{i_{0}},-N_{i_{0}}\right) u v_{+}=4\left(M_{i_{0}} \lambda\left(\mathbf{c}_{\mathbf{1}}\right)+N_{i_{0}} \lambda\left(\mathbf{c}_{\mathbf{2}}\right)\right) u_{i} v_{+} \in N
$$

$\operatorname{Logo}\left(u-h\left(M_{i_{0}}, N_{i_{0}}\right) u_{i_{0}}\right) v_{+} \in N$ e o número de somandos em $\left(u-h\left(M_{i_{0}}, N_{i_{0}}\right) u_{i_{0}}\right) v_{+} \in N$ é menor que $|I|$. Aplicando a hipótese de indução sobre $\left(u-h\left(M_{i_{0}}, N_{i_{0}}\right) u_{i_{0}}\right) v_{+}$, obtemos o resultado desejado para este caso.

Caso 2: Suponha que alguns $\left(M_{i}, N_{i}\right) \in S_{1}$ e os demais $\left(M_{j}, N_{j}\right) \in S_{2}$. Seja $h\left(M_{j_{0}}, N_{j_{0}}\right)$ o elemento mimimal entre os $h\left(M_{j}, N_{j}\right) \operatorname{com} h\left(M_{j}, N_{j}\right) \in S_{2}$. Considere $y=h\left(-\left[M_{j_{0}} / 2\right]+\right.$ $\left.a,-\left[N_{j_{0}} / 2\right]+b\right)$ tal que $a, b=0$ ou 1 de forma que $\left(-\left[M_{j_{0}} / 2\right]+a,-\left[N_{j_{0}} / 2\right]+b\right) \in S_{1}$. Então $\left[y, h\left(M_{i}, N_{i}\right)\right]=0 \mathrm{e}$

$$
\left[y, h\left(M_{j}, N_{j}\right)\right]=k_{j} h\left(-\left[M_{j_{0}} / 2\right]+a+M_{j},-\left[N_{j_{0}} / 2\right]+b+N_{j}\right) \neq 0,
$$

$\operatorname{com}\left(-\left[M_{j_{0}} / 2\right]+a+M_{j},-\left[N_{j_{0}} / 2\right]+b+N_{j}\right) \in S^{\perp} . \operatorname{Logo}$

$$
0 \neq y u v_{+}=\sum_{j \in I^{\prime}}\left[y, h\left(M_{j}, N_{j}\right)\right] u_{i} v_{+}
$$

onde $I^{\prime}$ é um subconjunto de $I$ e temos uma situação como no caso 1.

Caso 3: Suponha que alguns $\left(M_{i}, N_{i}\right) \in S^{\perp}$ e os demais $\left(M_{j}, N_{j}\right) \in S_{0}$. Seja $h\left(M_{j_{0}}, N_{j_{0}}\right)$ o elemento mimimal entre os $h\left(M_{j}, N_{j}\right) \operatorname{com} h\left(M_{j}, N_{j}\right) \in S_{0}$. Consideramos $y=h\left(-\left[M_{j_{0}} / 2\right]+\right.$ $\left.a,-\left[N_{j_{0}} / 2\right]+b\right)$ tal que $a, b=0$ ou 1 de forma que $\left(-\left[M_{j_{0}} / 2\right]+a,-\left[N_{j_{0}} / 2\right]+b\right) \in S^{\perp}$. Então $\left[y, h\left(M_{i}, N_{i}\right)\right]=0 \mathrm{e}$

$$
\left[y, h\left(M_{j}, N_{j}\right)\right]=k_{j} h\left(-\left[M_{j_{0}} / 2\right]+a+M_{j},-\left[N_{j_{0}} / 2\right]+b+N_{j}\right) \neq 0
$$

$\operatorname{com}\left(-\left[M_{j_{0}} / 2\right]+a+M_{j},-\left[N_{j_{0}} / 2\right]+b+N_{j}\right) \in S^{\perp}$. Logo

$$
0 \neq y u v_{+}=\sum_{j \in I^{\prime}}\left[y, h\left(M_{j}, N_{j}\right)\right] u_{i} v_{+}
$$


com $I^{\prime} \subset I$ e temos uma situação como no caso 1 .

Caso 4: Suponha que alguns $\left(M_{i}, N_{i}\right) \in S_{1} \cup S_{2}$ e os demais $\left(M_{j}, N_{j}\right) \in S_{0}$ (ou $\left.S^{\perp}\right)$. Neste caso, fixamos um $j_{0} \in I$ tal que $\left(M_{j_{0}}, N_{j_{0}}\right) \in S_{0}$ (ou $S^{\perp}$ ) e consideramos o elemento $y=h\left(-M_{j_{0}},-N_{j_{0}}\right)$. Então $0 \neq y u v_{+}=-4\left(M_{j_{0}} \lambda\left(\mathbf{c}_{\mathbf{1}}\right)+N_{j_{0}} \lambda\left(\mathbf{c}_{\mathbf{2}}\right)\right) u_{j_{0}} v_{+} \in N$ e aplicamos a hipótese de indução sobre o elemento $\left(u-h\left(-M_{j_{0}},-N_{j_{0}}\right) u_{j_{0}}\right) v_{+} \in N$.

Caso 5: Suponha que existem $\left(M_{i}, N_{i}\right) \in S_{1},\left(M_{j}, N_{j}\right) \in S_{2},\left(M_{k}, N_{k}\right) \in S_{0}$ e $\left(M_{l}, N_{l}\right) \in$ $S^{\perp}$. Consideramos o elemento minimal $\left(M_{q}, N_{q}\right)$ entre os $\left(M_{j}, N_{j}\right)$ e $\left(M_{l}, N_{l}\right)$

Considero $y=h\left(-\left[M_{q} / 2\right]+a,-\left[N_{q} / 2\right]+b\right)$ tal que $a, b=0$ ou 1 de forma que $\left(-\left[M_{q} / 2\right]+a,-\left[N_{q} / 2\right]+b\right) \in S_{1}$. Então

$$
\begin{gathered}
{\left[y, h\left(M_{i}, N_{i}\right)\right]=0, \quad\left[y, h\left(M_{k}, N_{k}\right)\right]=0,} \\
{\left[y, h\left(M_{j}, N_{j}\right)\right]=k_{j} h\left(-\left[M_{q} / 2\right]+a+M_{j},-\left[N_{q} / 2\right]+b+N_{j}\right) \neq 0,}
\end{gathered}
$$

$\operatorname{com}\left(-\left[M_{q} / 2\right]+a+M_{j},-\left[N_{q} / 2\right]+b+N_{j}\right) \in S^{\perp} \mathrm{e}$

$$
\left[y, h\left(M_{k}, N_{k}\right)\right]=k_{k} h\left(-\left[M_{q} / 2\right]+a+M_{k},-\left[N_{q} / 2\right]+b+N_{k}\right) \neq 0,
$$

$\operatorname{com}\left(-\left[M_{q} / 2\right]+a+M_{k},-\left[N_{q} / 2\right]+b+N_{k}\right) \in S_{2} . \operatorname{Logo}$

$$
0 \neq y u v_{+}=\sum_{j \in I^{\prime}}\left[y, h\left(M_{j}, N_{j}\right)\right] u_{i} v_{+},
$$

onde $I^{\prime}$ é um subconjunto de $I$ e temos uma situação como no caso 4.

Observação 4.3.3. Observamos que a condição $m \lambda\left(\mathbf{c}_{\mathbf{1}}\right)+n \lambda\left(\mathbf{c}_{\mathbf{2}}\right) \neq 0$ não é necessária para todo $(m, n) \in \mathbb{Z}^{2}$, mas para alguns $(m, n)$ específicos, como fica claro na demonstração do lema.

Teorema 4.3.4. Seja $N$ um submódulo de $M\{\lambda\}$. Se $\lambda\left(\mathbf{c}_{\mathbf{1}}\right) \neq 0$ ou $\lambda\left(\mathbf{c}_{\mathbf{2}}\right) \neq 0$ então

$$
N \simeq U\left(\mathcal{L}_{-2}\right) \otimes_{\mathbb{C}} \tilde{N}
$$

Prova: Seja $u v_{+} \in N$, com $u \in U\left(\mathcal{L}_{-}\right)$e podemos assumir, sem perda de generalidade, que $u$ é um elemento homogêneo. Então $u=\sum_{i \in I} u_{i} u_{i}^{\prime}$, com

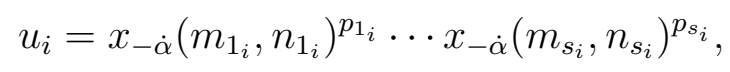


onde $x_{-\dot{\alpha}}\left(m_{1_{i}}, n_{1_{i}}\right)<\cdots<x_{-\dot{\alpha}}\left(m_{s_{i}}, n_{s_{i}}\right)$ e os $u_{i}$ são linearmente independentes.

Queremos mostrar, por indução sobre $h t(u)$, que $u_{i}^{\prime} v_{+} \in N$, para todo $i \in I$.

Supondo que $h t(u)=1$, temos

$$
u v_{+}=\sum_{i \in I} x_{-\dot{\alpha}}\left(m_{i}, n_{i}\right) u_{i}^{\prime} v_{+}
$$

Escolha o elemento $z=x_{\alpha}(-M,-N)$ tal que $(-M,-N) \in S, M, N>>0$ e $h(-M+$ $\left.m_{i},-N+n_{i}\right) u_{i}^{\prime} v_{+}$satisfaça as condições do Lema 4.3.2. Isto é possível pois, por hipótese, $\lambda\left(\mathbf{c}_{1}\right) \neq 0$ ou $\lambda\left(\mathbf{c}_{\mathbf{2}}\right) \neq 0$. Aplicando $z$ a $u v_{+}$temos

$$
z u v_{+}=\sum_{i \in I} t_{i} h\left(m_{i}-M, n_{i}-N\right) u_{i}^{\prime} v_{+}
$$

$\operatorname{com} t_{i} \in\{ \pm 1\}$. Como os $x_{-\dot{\alpha}}\left(m_{i}, n_{i}\right)$ são linearmente independentes os $h\left(m_{i}-M, n_{i}-N\right)$ também o são. $\operatorname{Logo} z u v_{+} \neq 0$. Aplicando o Lema 4.3.2 ao elemento $z u v_{+}$, obtemos o resultado desejado para este caso.

Agora suponhamos que $h t(u)>1$ e assumimos que o resultado se verifica para todo elemento homogêneo em $N$, com altura menor do que $h t(u)$. Seja $u=\sum_{i \in I} u_{i} u_{i}^{\prime}$, com

$$
u_{i}=x_{-\dot{\alpha}}\left(m_{1_{i}}, n_{1_{i}}\right)^{p_{1_{i}}} \cdots x_{-\dot{\alpha}}\left(m_{s_{i}}, n_{s_{i}}\right)^{p_{s_{i}}}
$$

onde $x_{-\dot{\alpha}}\left(m_{1_{i}}, n_{1_{i}}\right)<\cdots<x_{-\dot{\alpha}}\left(m_{s_{i}}, n_{s_{i}}\right)$ e os $u_{i}{ }^{6} s$ são linearmente independentes.

Como na demonstração da Proposição 4.3.1, escolhemos o elemento $z \in U(\mathcal{L}(J))$, $z=x_{\dot{\alpha}}(-M,-N) \operatorname{com}(-M,-N) \in S, M, N>>0$ tal que $h\left(-M+m_{s_{i}},-N+n_{s_{i}}\right) u_{i}^{\prime} v_{+}$ satisfaça as condições do Lema 4.3.2. Na proposição mostramos que $z u v_{+} \neq 0$ e o novo elemento será da forma

$$
z u v_{+}=\sum_{j \in J} q_{j} v_{j} h\left(m_{j}^{\prime}, n_{j}^{\prime}\right) v_{j}^{\prime} v_{+},
$$

onde $J$ é um novo conjunto de índices, possivelmente maior do que $I, q_{j} \in \mathbb{C}^{*}, v_{j} \in U\left(\mathcal{L}_{-2}\right)$, $\left(m_{j}^{\prime}, n_{j}^{\prime}\right)$ são combinções $\mathbb{Z}$-lineares de alguns $\left(m_{l_{i}}, n_{l_{i}}\right), i \in I$ e $l=1_{i}, \cdots, s_{i}$ e $v_{j}^{\prime}=u_{i}^{\prime}$ para algum $i \in I$.

Como $h t(z u)<h t(u)$, podemos aplicar a hipótese de indução sobre $z u v_{+}$e obter que $h\left(m_{j}^{\prime}, n_{j}^{\prime}\right) u_{j}^{\prime} v_{+} \in N$. Mas, como vimos na demonstração da Proposiçao 4.3.1, um dos $h\left(m_{j}^{\prime}, n_{j}^{\prime}\right) u_{j}^{\prime} v_{+}$é $\left[x_{\dot{\alpha}}(-M,-N), x_{-\dot{\alpha}}\left(m_{s_{a}}, n_{s_{a}}\right)\right] u_{a}^{\prime} v_{+}$quando $u_{a}$ é maximal entre os $u_{i}$. 
Assim

$$
\begin{aligned}
& {\left[x_{\dot{\alpha}}(-M,-N), x_{-\dot{\alpha}}\left(m_{s_{a}}, n_{s_{a}}\right)\right] u_{a}^{\prime} v_{+}} \\
& \quad=k h\left(-M+m_{s_{a}},-N+n_{s_{a}}\right) u_{a}^{\prime} v_{+} \in N
\end{aligned}
$$

com $k \in\{ \pm 1\}$. Aplicando o Lema 4.3.2 ao elemento acima obtemos $u_{a}^{\prime} v_{+}$. Completamos a prova por indução sobre a $|I|$ para o elemento $\left(u-u_{a} u_{a}^{\prime}\right) v_{+} \in N$.

Teorema 4.3.5. (i) Se $\tilde{M}$ é um G-módulo irredutível, então $M\{\lambda\}$ é um $\mathcal{L}(J)$-módulo irredutivel.

(ii) Se $\lambda\left(\mathbf{c}_{\mathbf{1}}\right) \neq 0$ ou $\lambda\left(\mathbf{c}_{\mathbf{2}}\right) \neq 0$ e $M\{\lambda\}$ é um $\mathcal{L}(J)$-módulo irredutivel, então $\tilde{M}$ é um G-módulo irredutível.

(iii) Se $\lambda\left(\mathbf{c}_{\mathbf{1}}\right)=\lambda\left(\mathbf{c}_{\mathbf{2}}\right)=0$, então ambos $\tilde{M}$ e $M\{\lambda\}$ são redutíveis.

Prova: (i) Segue do Lema 4.3.1.

(ii) Suponhamos que $\tilde{N}$ seja um submódulo próprio de $\tilde{M}$. Então $\{0\} \neq U(\mathcal{L}(J)) \tilde{N}$ é um submódulo de $M\{\lambda\}$. Como $M\{\lambda\}$ é irredutível temos $U(\mathcal{L}(J)) \tilde{N}=M\{\lambda\}$. Pelo Teorema 4.3.2, temos $U(\mathcal{L}(J)) \tilde{N}=U\left(\mathcal{L}_{-2}\right) \otimes \tilde{N}$, ou seja, $\tilde{N}=\tilde{M}$, contradição. Logo $\tilde{M}$ é irredutível.

(iii) A condição $\lambda\left(\mathbf{c}_{\mathbf{1}}\right)=\lambda\left(\mathbf{c}_{\mathbf{2}}\right)=0$ implica que $\tilde{M}$ é redutível. Logo $\tilde{M}$ possuirá um submódulo próprio que irá gerar um submódulo próprio de $M\{\lambda\}$.

\subsection{Considerações finais}

Observamos que $G^{\prime}=G_{1} \oplus G_{-1} \oplus \mathbb{C c}_{\mathbf{1}} \oplus \mathbb{C d}_{\mathbf{1}}$ é um subálgebra de $G$, que é a álgebra de Heisenberg com decomposição triangular $G^{\prime}=G_{1} \oplus G_{0}^{\prime} \oplus G_{-1}$, onde $G_{0}^{\prime}=\mathbb{C c}_{\mathbf{1}} \oplus \mathbb{C d}_{\mathbf{1}}$.

Para $\tau \in G_{0}^{*}$ denotamos por $\tilde{\tau}$ a restrição de $\tau$ a $\left(G_{0}^{\prime}\right)^{*}$. Seja

$$
M\{\tilde{\tau}\}=U\left(G^{\prime}\right) \otimes_{U\left(G_{1}+G_{0}^{\prime}\right)} \mathbb{C} v_{+}
$$

o módulo de Verma sobre $G^{\prime}$ induzido pelo $G_{1}+G_{0}^{\prime}$-módulo unidimensional cuja ação de $G_{1}+G_{0}^{\prime}$ é dada por $(h+x) v_{+}=\tilde{\tau}(h) v_{+}$, para todo $h \in G_{0}^{\prime}$ e $x \in G_{1}$.

Como $M\{\tau\} \simeq U\left(G_{-2}\right) \otimes M\{\tilde{\tau}\}$, se $M\{\tau\}$ é um $G$-módulo irredutível então $M\{\tilde{\tau}\}$ é um $G^{\prime}$-módulo irredutível e $\tau\left(\mathbf{c}_{\mathbf{1}}\right) \neq 0$. 
A álgebra $G$ tem semelhanças com a chamada álgebra Virasoro-like. Baseado em [20] acreditamos que a inversa da afirmação acima seja verdadeira para $G$, isto é, se $\tau\left(\mathbf{c}_{\mathbf{1}}\right) \neq 0$, então $M\{\tau\}$ é um $G$-módulo irredutível.

Estudamos nessa tese 2 tipos de módulos de Verma para a álgebra TKK afim estendida obtida de um semi-reticulado do $\mathbb{R}^{2}$ : o clássico e o imaginário. Acreditamos que os outros 2 casos intermediários podem ser reduzidos a estes usando as idéas do trabalho de Dimitrov, Futorny e Penkov [6]. Mas esta afimação e a do parágrafo anterior serão assuntos para os próximos estudos. 


\section{Referências Bibliográficas}

[1] S. Azam, Extended affine Weyl groups. Journal of Algebra, 1999, 214, 571-624.

[2] B. Allison, S. Azam, S. Berman, Y. Gao, A. Pianzola, Extended Affine Lie Algebras and Their Root Systems. Memoirs of the AMS, 1997, 126 (603).

[3] S. Berman, B. Cox, Enveloping algebras and representations of toroidal Lie algebras. Pacific J. Math. 1994, 165, 239-269.

[4] B. Cox, V. Futorny, Borel subalgebras and categories of highest weight modules for toroidal Lie algebras. Journal of Algebra 2001, 236, 1-28.

[5] M. Dokuchaev, L. M. Figueiredo, V. Futorny, Representations of the extended affine Lie algebra $\operatorname{sl}_{2}\left(\mathbb{C}_{q}\right)$. Commun. in Algebra, 2003, 31, 299-318.

[6] I. Dimitrov, V. Futorny, I. Penkov, A reduction theorem for highest weight modules over toroidal Lie algebras, Commun. in Math. Phys., 2004, 205, 47-63.

[7] V. Futorny, IThe parabolic subsets and corresponding representations of affine Lie algebras, Contemporary Mathematics (2), 1992, 131, 45-52.

[8] V. Futorny, I. Kashuba, Verma type modules for toroidal Lie algebras. Commun. in Algebra, 1999, 27 (8), 3979-3991.

[9] R. Hoegh-Krohn, B. Torresani, Classification and construction of quasisimples Lie algebras. J. Functional Anal., 1990, 89, 106-136.

[10] J.E. Humphreys, Introduction to Lie algebras and representation theory. Springer, 1972. 
[11] N. Jacobson, Structure and representations of Jordan algebras. American Mathematical Society, 1968, 1-56, 307-333.

[12] H.P. Jakobsen, V.G. Kac, A new class of unitarizable highest weight representations of infinite-dimensional Lie algebras. Lecture Notes in Physics, 1985, 226, 1-20.

[13] V. Kac, Infinite-dimensional Lie algebras. Cambridge University Press, 1990.

[14] R. Moody, A. Pianzola, Lie algebras with triangular decompositions. Canadian Mathematical Society Series of Monographs and Advanced Texts, 1995, 28-49, 91-128.

[15] R. Moody, S. E. Rao, T. Yokonuma, Toroidal Lie algebras and vertex representations. Geom. Ded., 1990, 35, 283-307.

[16] A. N. Nashimoto, Álgebras de Lie afins estendidas do tipo $A_{1}$, (Dissertação de Mestrado, Universidade de São Paulo, 2002).

[17] E. Neher, Generators and relations for 3-graded Lie algebras. Journal of Algebra, 1993, 155, 1-35.

[18] K. Saito, Extended affine root systems, 1 (coxeter transformations), RIMS, Kyoto Univ., 1985, 21, 75-179.

[19] S. Tan, TKK-algebras and vertex operator representation. Journal of Algebra, 1999, 211, 298-342.

[20] X.D. Wang, K. Zhao, Verma modules over Virasoro-like algebras. J. Aust. Math. Soc., 2006, 80, 179-191.

[21] Y. Yoshii, Coordinate algebras of extended affine Lie algebras of type $A_{1}$. Journal of Algebra, 2000, 234, 128-168. 


\section{Índice Remissivo}

álgebra TKK afim estendida, 19

conjunto positivo de raízes isotrópicas, 25

construção de Tits-Kantor-Koecher, 15

grupo de Weyl afim estendido, 11

módulo de Verma clássico, 34

módulo de Verma imaginário, 57

módulo integrável, 49

módulo tipo Verma, 34, 57

reticulado, 14

semi-reticulado, 13

sistema de raízes afim estendido, 9

sistema de raízes positivo normal, 26

subálgebra de Borel, 34 